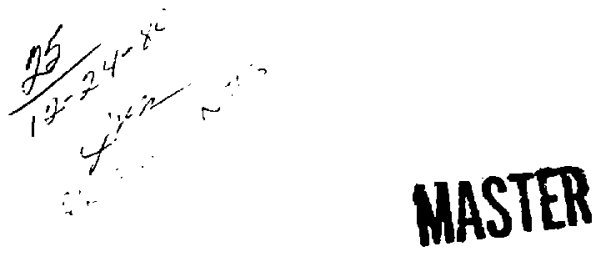

\title{
Conceptual Design of a 1.5-MJ, 2-Hz KrF Fusion Laser System
}

\author{
J. Caird, W. O. Ailen, and H. G. Hipkin \\ Bechtel National, Inc., San Francisco, Calif.
}

J. Benford, Y. G. Chen, D. Dakin, G. Frazier, T. Nafi, and T. S. T. Young Physics International Co., San Leandro, Calif.

M. R. Flannery, G. M. Perron, S. N. Suchard, and D. E. Vandenburg Hughes Aircraft Co., Los Angeles, Calif.

E. V. George, R. A. Haas, W. F. Krupke, L. Pleasaice, and L. Seppala Lawrence Livermore National Laboratory, Livermore, Calif.

\section{December 1980}

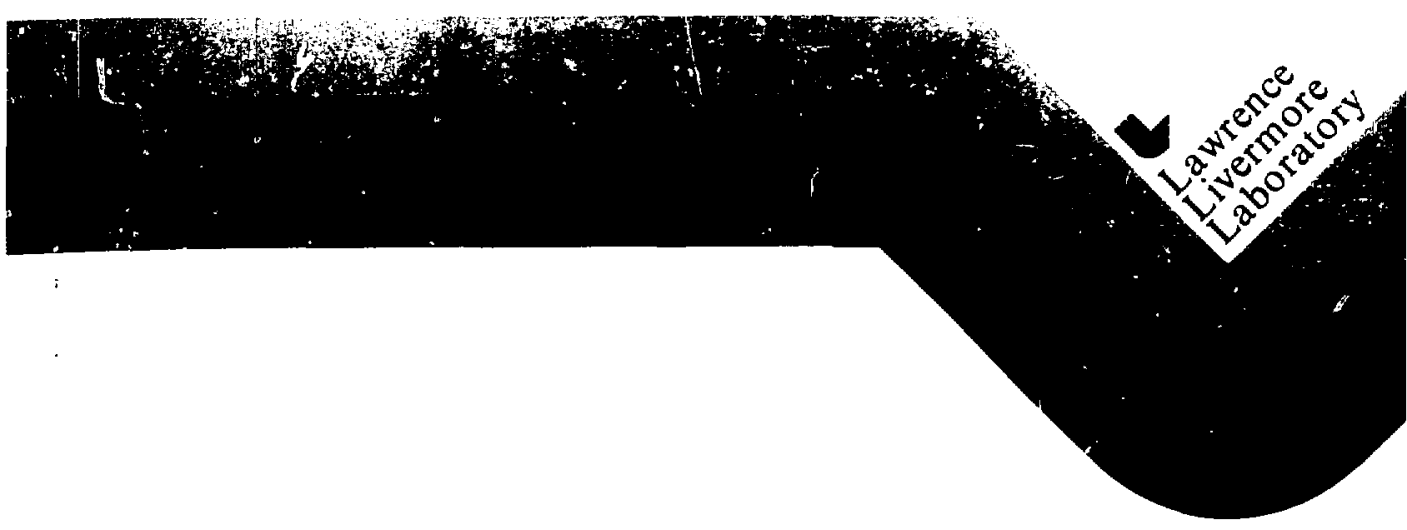




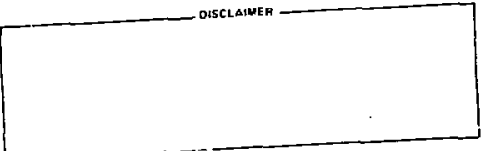

\section{Conceptual Design of a 1.5-MJ, 2-Hz KrF Fusion Laser System}

J. Caird, W. O. Allen, and H. G. Hipkin Bechtel National, Inc., San Francisco, Calif.

J. Benford, Y. G. Chen, D. Dakin, G. Frazier, T. Naff, and T. S. T. Young $\therefore$ - Physics International Co., San Legndro, Calif.

M. R. Flannery, G. M. Perron, S. N. Suchard, and D. E. Vandenburg Hughes Aireraft Co., Los Angeles, Calif.

E. V. George, R. A. Haas, W. F. Krupke, L. Pleasance, and L. Seppala Lawrence Livermore National Laboratory, Livermore, Calif.

Manuscript date: December 1980

LAWRENCE LIVERMORE LABORATORY

University of California $\bullet$ Livermore, California $\bullet 94550$ 
Table of Contents

\section{Section}

Page

Figures

vi

Tables

vi1

I. Introduction

II. Overview of Laser-System Design

1. Laser-System Architecture and Building

Configuration

2. KrF Amplifier Performance Characteristics 10

3. Raman Compressor Derformance Characteristics 10

4. E-Beam Pulsed Power 10

5. Optical Systen Design 12

6. Rey Technology Development Issues 16

7. Summary of Laser-System Cost and Efficiency 17

8. References 21

III. Technical Sumary: Appendices

Appendix 1. E-Beams and Prised-Power Conditioning 1-i

Figures

$1-\mathbf{i} i$

Tables

1-i i i

1.1 Pulsed-Power Options I-1

1.2 System Designs 1-3

1.2.1 Pulse-Forming Line System 1-3

1.2.2 Pulse-Porming Network System 1-10

1.2.3 Diode and Magnetic Field Coils 1-13

1.2.3.1 General Diode parameters i-13

1.2.3.2 Beam Deposition 1-10

1.2.3,3 Magnetic Field Coils 1-17

1.2.3.4 Diode Modularization 1-18

1.3 Cost. Structure
$1-20$

1.4 Technology-Development Issues 1 -22

1.5 Conclusions 1-24

$\begin{array}{ll}1.6 \text { References } & \text { 1-25 }\end{array}$ 


\section{Table of Contents (continued)}

Appendix 2. Optical Systems 2-i

Figures

2-iii

Tables

2-i i i

2.1 Introduction

2.2 System Options 2-2

2.2.1 System Architect:1re 2-6

2.2.2 Mirror Construction 2-7

2.2.3 Mitror-Mounting strategy 2-7

2.2.4 Mirror-Aligment Strategy 2-8

2.2.5 $\because \mathrm{rF}$ Amplifier Windows 2-9

2.2.6 Beam-Path Enclosure 2-9

2.2.7 Beam-Path Gas 2-9

2.2.8 Raman-Cell Windows 2-9

2.3 Description of the Baseline Design 2-9

2.4 Cost scaling 2-13

2.5 Technology Development 2-15

2.6 Conclusions 2-20

Appendix 3. Process Systems, Facilities, and Capital Cost 3-i

Figures 3-iv

Tables 3-v

3.1 Introduction and Summary 3-1

3.2 RrF Amplifier Gas Flow Systems 3-3

3.2.1 General Considerations 3-3

$3.2 .2 \mathrm{KrF}$ Amplifier Window 3-6

3.2,2.1 Considerations for a Solid window 3-8

3.2.2.2 Considerations for a Purely

Aerodynamic window 3-11

3.2.2.3 Considerations for a Gas

Buffered Window

3-11 
Table of contents (continued)

Appendix

Page

3.2.3 Fluorine Seperation systems 3-12

3.2.3.1 Crvogenic Fractionation 3-13

3.2.3.2 Separation by Absorption 3-18

3.2 .3 .3 Chemlat Separdion $3-1$ B

3.2. 3.4 conclision on $\mathrm{munrine}$ Separation 3-22

3.2.4 other flow new are risiderations 3-22

3. 2.4.1 monstruction kiacerials 3-23

3. 2.4.? Hat Reprova! 3-23

$3 . \therefore 4.4$ pressure-wave Damping and Flow

Management 3-24

?.2.4.4 prestsern urops and Elower Prwar 3-25

3.2.4.5 inoling vatar Hlow Power 3-26

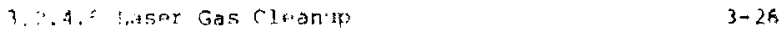

3.7.4.7 7itar: in \$nd shutrown operations 3-27

3.2.t.9 Flow Sister fidyot 3-2E

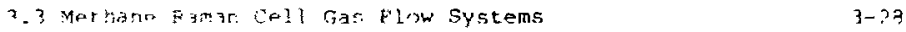

3.4 Mver 7l: S\%erm Enecay Outplt and Efficiency 3-32

3.5 Structuesia and Auxiliary Equipment 3-33

3.5. 1 5tarker Beam Channcis jusign 3-33

3. 5.2 perimeter Amplifier sustem Housing $3-.6$

3.5.3 Fut Diliding Design 3-39

3.5.4 Raman Compressor Building Design 3-40

3.5.5 sitework 3-4l

3.5 Facility Construction Cost Estimate 3-41

3.' I General 3-41

3.6.2 Pricing beveis 3-42

3.6 .3 Direct Facilicy Construction costs 3-42

$3 . t_{3} 3.1$ Mechanical Equipment $\quad 3-42$

3.5.3.2 Matetials 3-43

3.6.3.3 Cons':uction tahor 3-43

3.6.4 Indirect Fiald Costs 3-43

3.6 .5 frone End Frorts $3-43$

3.6.6 Hone $04 f$ ice Services $3-43$ 
Table of Contents (continued)

Appendix

3.6.7 Contingency

3-45

3.5.8 Design Assumptions

3.6.9 Exrlusions

3.6.10 Estimate Summary (400-ns pulsed power)

3.6. 11 900-ns System Costs

3. 7 Summar'y of Technol ogy Development Issues

3.7.1 ETF Des:gr: Prilosophy

3.7.2 KrF Final Amplifier Design

3.7.3 Optigal Sus*..r Design

3.7 .4 PIlsed-Pnwer Systam Design

3.7 .5 Other Facilitv Isejes

Figures

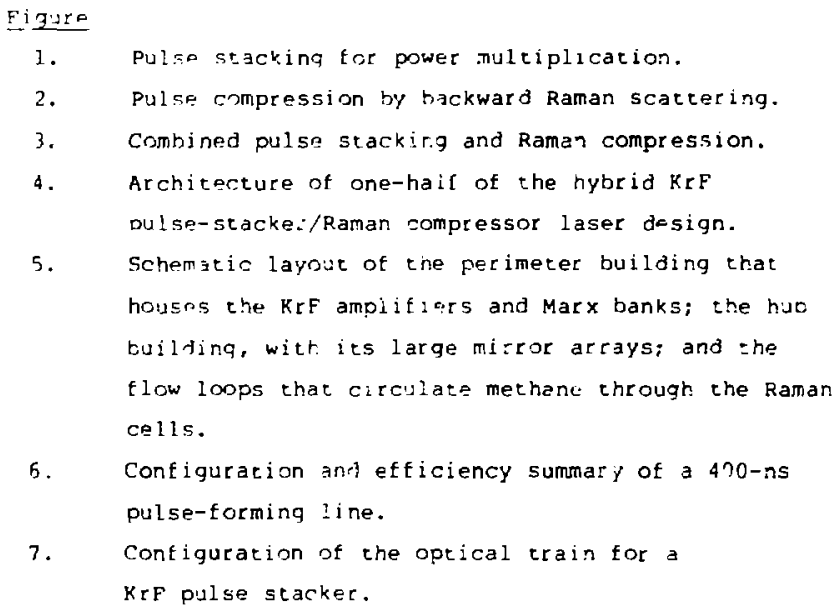
housos the KrF ampilf 1 ars and Marx banks; the huo bullting, witt its large mirror arrays; and the flow loops that circulate methenc through the Raman ce $11 \mathrm{~s}$.

5. Configuration and efficiency summary of a 400-ns pulse-forming line.

7. Contiguration of the optical train for a KrF pulse stacker. 


\section{Tables}

Table

Page

1. Nominal performance specifications for each RrF final amplifier.

2. Nominal performance specifications for Raman compressor using methane.

3. Laser-svstem output and efficiency (400-ns pulsed power).

4. Stacker-compressor laser-system costs (400-ns pulsed power). 


\section{Introduction}

Current projections suggest that the future application of laser fusion to power generation will require a short-wavelength laser (from visible to ultraviclet) that will be able to deliver 5- to $20-n s$ pulses at one or two hundred terawatts with a pulse rate of a few hertz and an overall efficiency greater than 58. An interest in applying recently developed ultraviolet excimer lasers to this problem has stimulated the present reexamination of optical pulse-compression tezhniques. In a sense, all lasers suggested for application to laser fusion are compressors that convert a large pump source at low energy density to a very intense pulse on a small target; however, most. of these lasers achieve a large part of the required latensity gain by storing energy in exc: ted states during a long excitation pulse and then releasing it to a short optical pulse some time later.

The new excimer lasers, such as the 248-nm KrF laser, cannot be operated in an energy-storage mode since they have high gain and short excited-state lifetimes ( $\leqslant 10 \mathrm{~ns}$ ); efficient operation of these lasers is possible only if energy is extracted during the entire duration of the pump pulse. The design r.t a pulser that can drive a large laser in a time much less than about $100 \mathrm{~ns}$ presents formidable difficulties; in addition, nonsaturable phocoabsorption losses within the KrF laser medium limit the output intensity of the laser, for efficient operation, to the range of 10 to $20 \mathrm{NW} / \mathrm{cm}^{2}$. Similar limitations exist for the other rare-gas-halide excimer lasers. Thus, even if short excitation pulses could be readily generated, the KrF amplifier output fluence would be only a fraction of a joule per square centimeter. Consequently, application of a nonstorage laser such as $K_{r F}$ to the generation of 5- to $20-\mathrm{ns}$ pulses requires an efficient method for converting krF pulses, which are longer than $100 \mathrm{~ns}$, to the shorter pulses desired. In addition, some optical means of intensifying the $\mathrm{KrF}$ laser radiation is usually required (especially at shorter pump-pulse lengths) to operate at laser output fluences close to damage limits, where optical area and cost are minimized. The potential advantages of an optical pulse compressor are considerable, since present projections of the efficiency of large $\mathrm{KrF}$ lasers lie in the range of 5 to 108 , and several other rare-gas-halide or metal-halide excimer lasers may offer similar high efficiencies. These lasers are the most efficient 
gas-phase lasers known at wavelengths shorter than the efficient molecular infrared transitions of $\mathrm{CO}_{2}, \mathrm{CO}$, and $\mathrm{EF}$.

One pulse compression approach uses energy extracted from an efficient $\mathrm{Kr} F$ laser amplifier, which is pumped for $100 \mathrm{~ns}$ or longer, to pump an energy-storing laser medium, such as atomic iodine or other media. A second conceptual approach, illustrated in Fig. 1, is to extract erergy from a $\mathrm{krF}$ amplifier using a sequence of short ( $10 \mathrm{~ns}$ ) pulses propagating through the amplifier at slightly different angles and different times with respect to each other. These angle-coded time-sequenced pulses could then be applied to the target simultaneously (i.e., pulse stacked) ${ }^{l}$ by adjusting the propagation time to the target for each pulse. Yet a third technique for e:-ifectively compressing a long ( $\geqslant 100 \mathrm{~ns})$ output pulse of an excimer laser is that of backward-wave haman scattering, which has been recently suggestad and subsequently validated scientif ically by Murray, et al. ${ }^{2}$ Figure?

illustrates the principle involved in optical pulse compression using a backward Raman amplifier. A long laser pulse of duration ' $p$ ' called the pump pulse, propagates from right to left into a cell containing a Raman active medium (interaction zone), producing gain at a frequency $\nu_{p}-\nu_{r}=$ $\nu_{s}$. Here, $\nu_{p}$ is the pump frequency, $\nu_{r}$ is the frequency of the Raman transition excited, and $\nu_{s}$ is called the first stokes frequency. A stokes pulse at $\nu_{s}$ is injected into the cell at the time when the leading edge of the pump pulse just starts to exit the end of the cell. As the Stokes pulse propagates from left to $r$ ight, and the pump pulse propagates from $r$ ight to left, the stokes pulse is amplified at the expense of the pump pulse. After a time $\tau_{p} / 2$, the Stokes pulse emerges from the cell as an amplified pulse traveling in a backward direction with respect to the pump pu'se. The Stokes pulse can be much shorter in time than the pump pulse, as shown in Fig. 2, so the result is to compress a long laser pulse at $\nu_{p}$ to a short pulse at $\nu_{s}$. There is a net loss of energy since $\nu_{s}: \nu_{p} ;$ also, part of the pump radiation may not be converted and, if not, will pass out through the exit window of the cell. The backward Raman amplifier can also be considered as an energy-storage laser, but one in which the energy is stored in an intense collimated pump beam of photons rather than in excited atoms or molecules. Gain and saturation can then te discussed in terms very similar to those used to describe more conventional energy-storage amplifiers. In recent studies, the two techniques of pulse stacking and Raman pulse compression have buen combined (Fig. 3) in an effort to realize efficient, scalable laser systems 


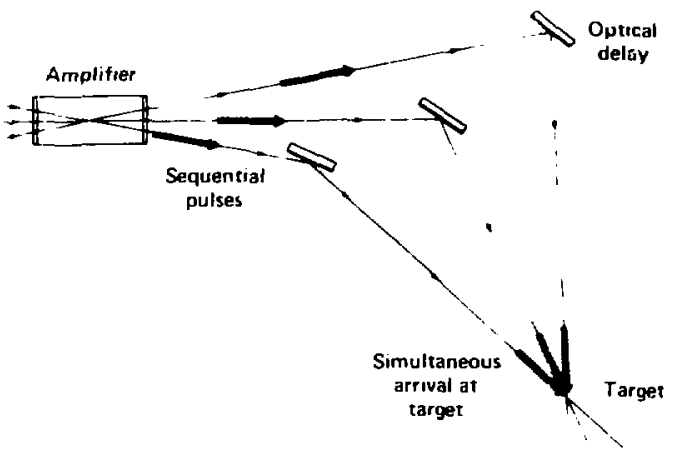

Fig. 1. Pulse stacking for power multıplication. 


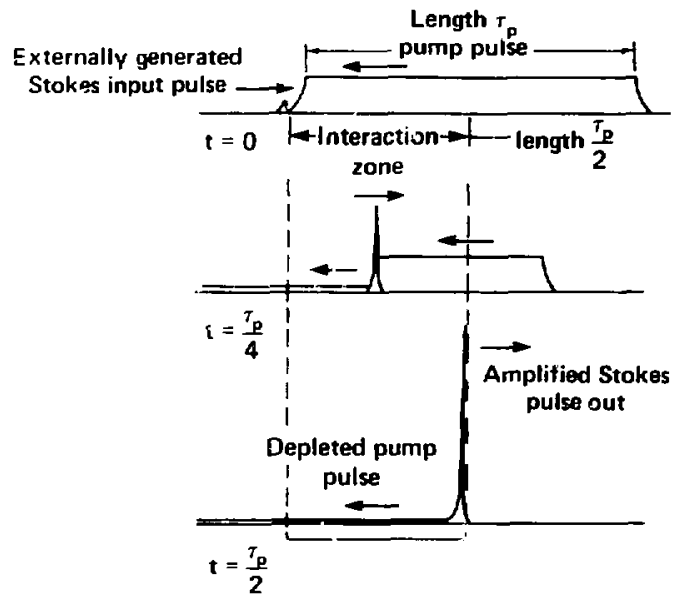

Fig. 2. Pilse compression by backward Raman scattering. 


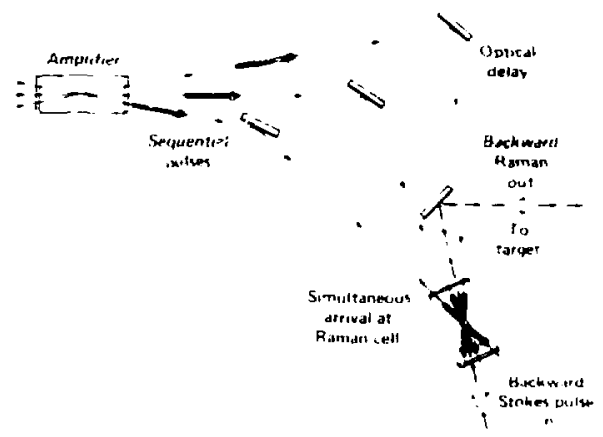

Fig. 3. Combined pulse stacking and Raman compression. 
with high overall compression factors that would minimize the physical and technological limitatıons of using each technique separately.

This report presents the results of a conceptual design study of a short-wavelength, repetitively pulsed laser system for use in civilian power-reneratior upplications. The hybrid pulse-stacker/Raman-compressor archicanture was chosen to compress a long (400 to $900 \mathrm{~ns}$ ) KrF laser pulse to the 20-ne, timescale required to drive a fusion target. The technical material presented in Refs. 1 and 2 was assumed as the point of departure for the conceptual design study.

The objeciive of the study was to identify and examine the key technical issues for short-wavelength fusion-laser technoloyy and to point out places where significant innovation or development is needed to reduce costs and technical risks. The approach was to perform a conceptua. design of a KrF fusion-laser system suitable for an experimental test facility (ETF), the filst repetitively pulsed fusion driver. We chose design goals that are representative of such a near-term facility: output energy of $1.5 \mathrm{MJ}$, repetition rate (rep-rate) of $2 \mathrm{~Hz}$, pulse lengti of $20 \mathrm{~ns}$, efficiency of 58 , and lifetime of $10^{7}$ shots. Choosing an ETF system allowed us to use hear-term technology to fcrm relatively concrete technology and cost assessments. Because of funding limitations $(\$ 40 \mathrm{~K} / \operatorname{contractor} \times 3$ contractors $=$ $\$ 120 \mathrm{~K}$ for the total effort), the scope of the study was restricted to an engineering assessment of a specific point design that appeared at the outset of the study to fullfill the design goals. Alternatives to the point design and further optimizations were only briefly considered. The results of the study ciear $l y$ indicate that further innovation and optimization of this laser-system concept are essential to make it compelling in the context of civilian commercial-power applications.

This conceptual design is the collaborative effort of Bechtel National, Inc., who did system integration, mechanical design, building, layout, and to:al laser-system costing; Physics International, who did power conditioning and $i$ ts costing; Hughes Aircraft Co., who did optical and al ignment systems and their costing; and Lawrence Livermore National Laboratory, who provided the Krf-amplifier and Raman-compressor performance parameters, the ETF design goaj.s, and overall direction. The report consists of two major elements: Section II presents an overview of the design study and discusses the major results and conclusions derived from this study; and section III provides details of the technical analyses and results and presents technical summaries 
that discuss e-beam pulsed-power conditioning, optical-system design, mechanical-systems design, facilities, overall laser-system effic:ency, and capital costs.

\section{Ir. Ow-rview of Laser-System Design}

\section{Laser-System Architezture nt Euilding Configuration}

The architecture or the hyt: 'd laser system is shown in rig. 4. We divide the Iaser into two sujsystems: the pulse stacker and the Raman compro -or. A KrF preamplifier and amplifier, nperating at $248 \mathrm{~nm}$, prepares $100-n s$ pulses that are Eed intr !; beam lines, ach $120 \mathrm{~m}$ long, that extend radially outward from a central hu builira. The $K r F$ mplifiers and pulse-forming lines are located at the enos or the beam liri $\vdots n$ a perimeter building. The laser pulses make three rount aps an the bean tuhes. In an input jelay line, they first reflect off, r staggered mirrors to produce a 400-ns crai, of four $100-n s$ pulses. Thes pulsne are then double-passed through the K-F ampifiat, which

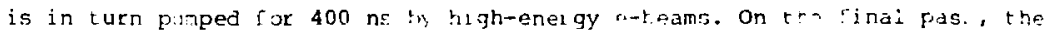
amplified pujses are incoherent $2 \because$ recombined into a side-by-side array of 100-ns pulser. The 14 arrays $0: 2 \times 2$ pulses, totaling $1.9 \mathrm{MJ}$, aro then simultaneou: ! directed from tro hub building through the Raman iompressor cell, which rontai as methine. in the meantime, a stokes pulso has been prepared thi. is $20 \mathrm{~ns}$ in rarption and has a wavelength of 268 rus. This Stokes pulse is counterpropagated thr. $y^{n}$ the 'aman coll and is aplifier! to the 1.5-MJ leve?. This final fivel id puise sompression is accomplisher in the Raman cell with $80 z$ coniersion efficienc $\%$.

A detailer viok of the builiding lays." is pro ald in Fig. 5 . The perimeter bul?ding contas ns il: KrF ampi ifiers, Marx banks, associated power supplies, gas cooling and circulation equipment, and energy stores for t:e guide magnets that are reyuirat for the uniform deposition of e-bear energy in the Y.rF medium. The hub hui? no contains the primul an tertury mirrors for all 14 amplifier chanricis and is the poirt wher: che las=r pulses are reassembled and pointed at the Romw romitessn: anils. The flrw lwops for circulating and cooling the nethane gas are shown. Brewster-angle windows of fused silica are used in the Ramar. cell'. The ont:re Raman tunn=: $15180 \mathrm{~m}$ long, to allow for sychrond dyule-passing. "sth the 100-ns KrF pump beams and the 20-ns stokse ream witrin each $82 \pi-$ wll. 


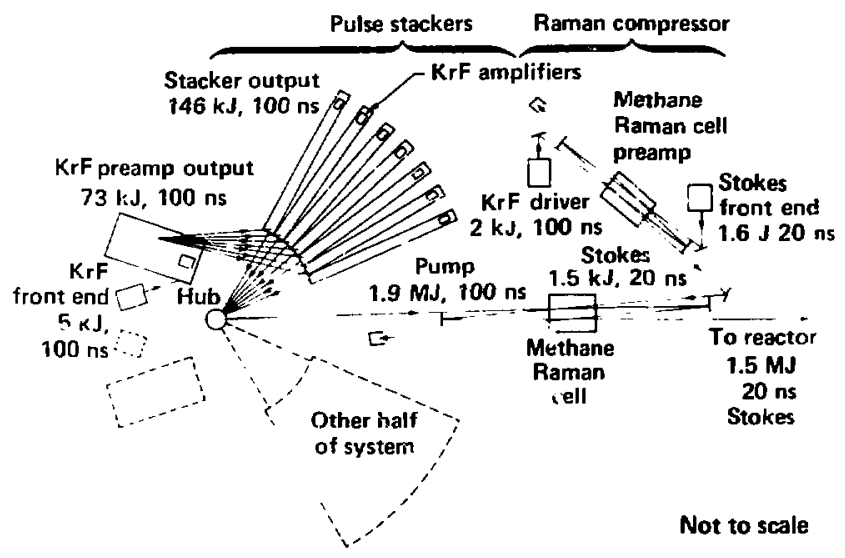

Fig. 4. Architecture of one-half of the hybrid KrF pulse-stacker/Raman compressor laser design. 


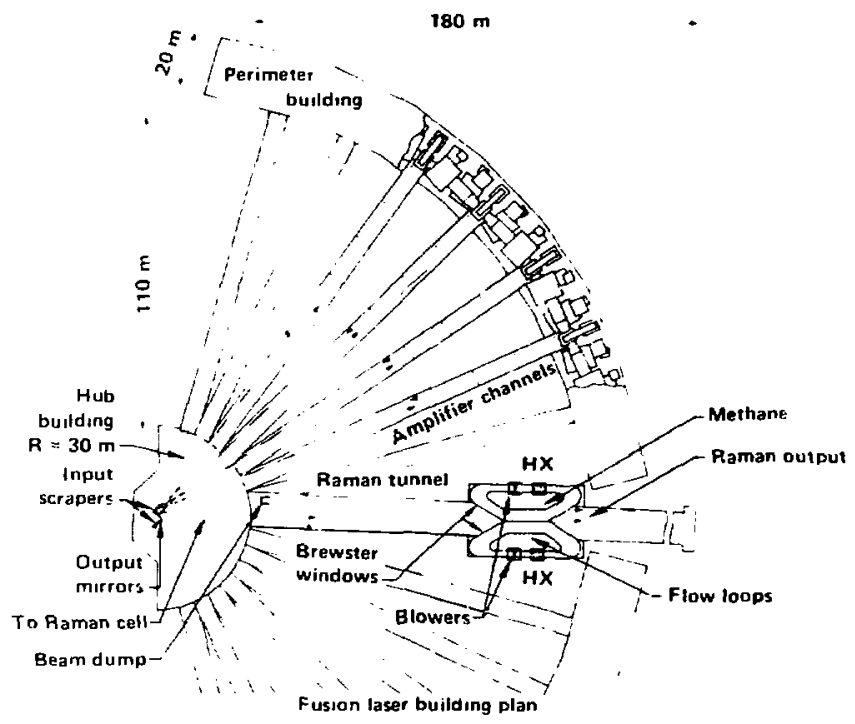

Fig. 5. Schematic layout of the perimeter building that houses the RrF amplifiers and Marx banks; the hub building, with its large mirror arrays; and the flow loops that circulate methane through the Raman cells. 


\section{KrF Amplifier Performance Characteristics}

The nominal performance specifications for the final RrF amplifiers are given in Table 1. These specifications were used during the study, although subsequent calculations are somewhat less optimistic. The output energy of 160 .J per $2 \times 2 \times 3$ in amplifier does not include assumed mirror losses (58 each), window losses (28 per surface) or fill factors (908), which were separately accounted for. The fi.sal amplifier has a nominal energy gain of 20. Each amplifier is equipped with rectangular magnetic-field coils, ea=.1 $5 \times 3.3 \mathrm{~m}$ in size and separated by $5 \mathrm{~m}$, that provide a 1-kG field in the active medium. This Helmholtz coil system gives 208 field uniformity and leads to 908 deposition efficiency. It requires $0.65 \mathrm{~mJ}$ of stored energy per pulse, transferred reso... Intly from a lo-kV capacitor bask. The $15 z$ energy loss estimated per shot leads to a rower requirement of $2.8 \mathrm{Mu}$ for all 14 amplifiers. In addition, $5.6 \mathrm{MH}$ of power are required for the gas circulation a!d cooling systems, which flow the gas at $8 \mathrm{~m} / \mathrm{s}$.

\section{Raman Compressor Performance Characteristics}

In Table 2, we give the nominal performance parameters for the $\mathrm{CH}_{4}$ Raman compressur, which must accomodate all 14 bear arzays. A length of $15 \mathrm{~m}$ of Raman scattering medium is required to efficiently deplete the 100-ns KrF pulse. Because of the high quantum efficiency of the Raman scattering in methane (939), there is little heat deposited, and only a slow flow of methane gas is required. The 1-atm cell pressure and the absence of large overpressures allow the use of a large expanse of fus-d-silica window panes, which are arranged in 2 × 7 arrays.

\section{E-Beam Pulsed Power}

For the e-beams and power conditioning, we examined both pulse-forming networks (PFN) and pulse-forming lines (PFL) in the range from 100-ns to 1- $\mu s$ pulse durations. For the 400-ns duration that was chosen, the PFL is preferable despite the fact that the energy must be stored twice (once in the Marx generator and again in the liquid pulse-forming linel and in spite of the requirement for a high-voltage output switch. At this pump-pulse duration, tho 
Table 1. Nominal. performance specifications for each KrF final amplifier.

$\begin{array}{ll}\text { Aperture } & 2 \times 2 \mathrm{~m} \\ \text { Length } & 3 \mathrm{\pi} \\ \text { Output fluence } & 4 \mathrm{~J} / \mathrm{cm}^{2} \\ \text { Intrinsic efficiencl } & 20 \mathrm{z} \\ \text { Pump time } & 400 \mathrm{~ns} \\ \text { E-beam energy } & 1.3 \mathrm{MeV} \\ \text { Amplifier output energy } & 260 \mathrm{~kJ} \\ \text { Stacker compression factor } & 4 \times \\ \text { Stacker cutput pulse time } & 100 \mathrm{~ns} \\ \text { Ar/Kr/F mixture } & 0.937 / 0.060 / 0.003 \mathrm{~atm}\end{array}$

Table 2. Nominal perfornance specifications for Raman compressor using methane.

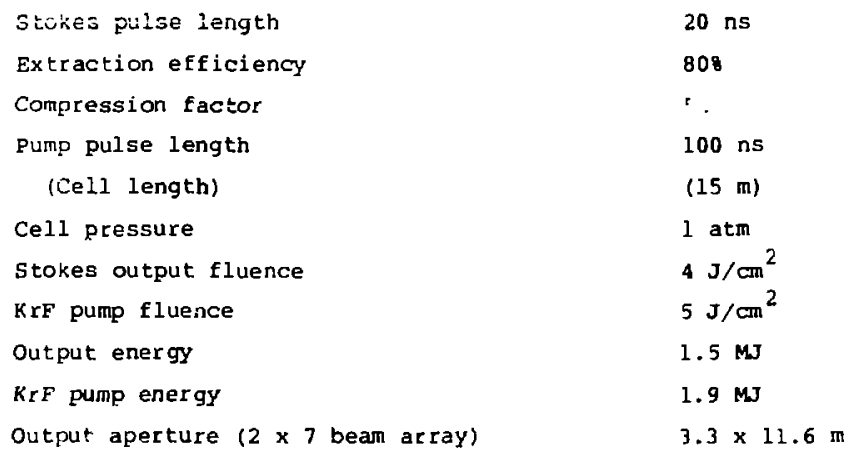


PFN is less attractive besause the number of parallel elements required to keep the inductance sufficiently low in a PFN increases with the inverse square of the pulse duration. As the number of Marx unit's increases, the inductance of connections rises with sufficient rapidity that no practical solution appears feasible at the 400-ns pulse length.

In the KrF laser system, there are 28 e-beams and PFLs, each of which is called a "side," since each KrF amplifier is punped fron two sides. In each PFL, the energy is first stored in eight oil-immersed Marx generators that

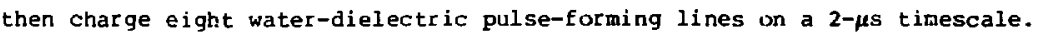
Each output line (OL) bifurcates, driving two diodes; therefore, each e-bean aperture, measuring $3 \times 2 \mathrm{~m}$, consists of 16 modular diodes, Euch side stores $1.3 \mathrm{MJ}$ (for a total of $36 \mathrm{MJ}$ stored energy per pulse) in $1.28-\mu \mathrm{F}, 50-\mathrm{kV}$ capacitors. The energy losses are given in Fig. 6, which also shows the relative sizes of components. The overall electrical system efficiency is 578 .

The system is designed to operate for $10^{7}$ shots with only routine maintenance, and the capacitor voltages are derated by a factor of two to attain this lifetime. The Marx switcines will erode about $100 \mathrm{~g}$ per switch in $10^{7}$ shots at an erosion rate of $50 \mu \mathrm{g} / \mathrm{C}$; changing the gas pressure during the liferime can compensate for widening of the gap.

The output switch for each PFL is gas insulated and command triggered, and it operates through six breakdown channels, each of which carrys 53 hA. Tinis switch is currently being developed.

A 900-ns PFN was also designed and costed for a 9-pulse stacker option, should the longer-pulse-length electrical system prove to be substantially less expen'ive, Unfortunately, we were not able to separately optimize the $\mathrm{Kr} F$ amplifier uesign and architecture for each pulse length. However, the cost per joule stored was practically identical for each design considered, leading to a selection of the 400-ns, four-pulse stacker on the basis of a les: costly optical system.

5. Optical System Design

The configuration of the optical system is the key to the pulse-compression scheme. To take advantage of the long-pulse-length $\mathrm{KrF}$ laser, we feed the final amplifier with an optical delay line that temporally stacks a sequence of $\mathrm{N}$ short pulses of length $;$ through the amplifier at slightly different angles. By slightly overfilling the amplifier with each pulse, we can extract 


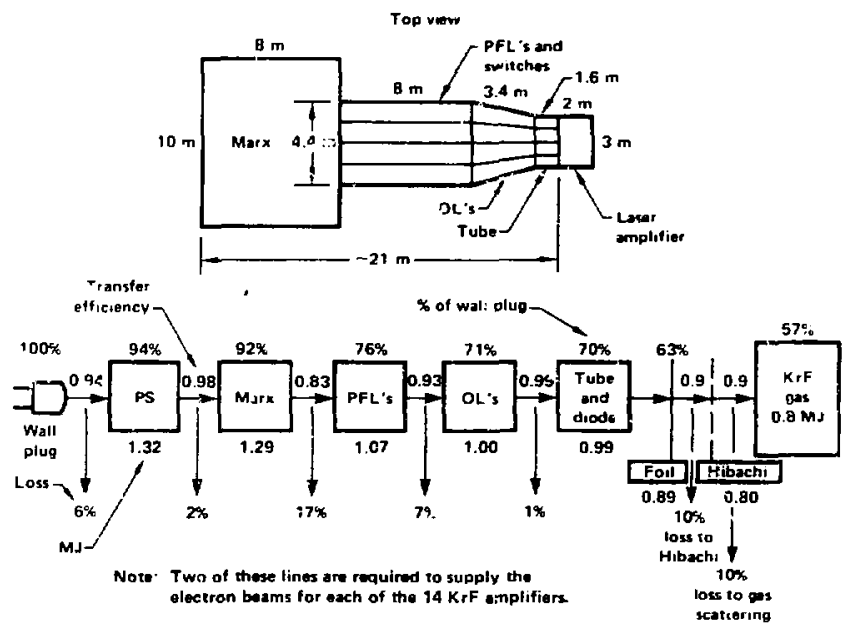

Fig. 6. Configuration and efficiency summary of a 400-ns pulse-forming line. 
the energy over the entire volume for a pulse length of $\mathrm{Nr}$, which allows efficient pumping of the amplifier by long-pulse electron beams. Angular multiplexing of the short pulses allows them to be separated spatially at the output of the amplifier, where their relative delays are renoved in a second optical-delay line. The pulses are reassembled into a spatial matrix rather than being superposed, since the latter would require very careful control of the interbeam coherence. The general architecture of this pulse stacker is shown in the perspective view of Fig. 7.

Further pulse compression, beam cleanup, and prepulse isolation are accomplished by using the $K r F$ laser as a pump for a double-pass backward Raman scattering cell filled with methane. This cell converts the 248-nm, 100-ns, $1.9-\mathrm{MJ}$ KrF pump pulse of moderate wavefront quality to a $268-\mathrm{nm}, 20-\mathrm{ns}, 1.5-\mathrm{MJ}$ Raman-shifted pulse with a smooth wavefront that has negligible prepulse energy .

Effective prepulse isolation is accomplished because there can be very little energy directed toward the pellet in the absence sf an input (at the Stokes frequency) that propagates counter to the RrF pimp beam in the Raman cell. The Raman input to the cell requires only $1.5 \mathrm{~W}$ to generate the $1.5-\mathrm{MJ}$ output, and this input in turn is produced in a front end that requires an input of only $1.6 \mathrm{~J}$. This millionfold gain accounts for the effective prepulse isolation control of the Raman cell. The high quality of the output wavefront is essentially independent of the guality of the pump wavefront.

Several design options were considered; e.g.. the 4-pulse vs a 9-pulse stacker, different types of mirror substrate, several alignment schemes, solid vs aerodynamic windows (aero windows), and different beam-path gases. The chasen baseline design is a compact four-beam stacker that minimizes the complaxity of the mirror mounts and aligrument system and that fits in a beam tube with a cross section of only $2.1 \times 6.2 \mathrm{~m}$.

An image-Alignment system was selected that uses laser diodes mounted or. each mirror array as point sources of light. Consider three sequential mirrors in the optical train $\left(M_{1}, M_{2}\right.$, and $\left.M_{3}\right)$. The light sources are mounted on the edges of mirror $M_{1}$, and a precision video camera, or $C C D$, is mounted on $M_{3}$. The mirror $M_{2}$ is aligned by looking at the reflected imacie in $M_{2}$ of the point sources located on the mirror $M_{1}$. The video camera on $M_{3}$ is used to compare the image w: th a reference perfect-alignment image, and an error signal is generated; a servomechanism drives the mirror mount until the actual and the reference images agree. The entire optical train is aligned by 


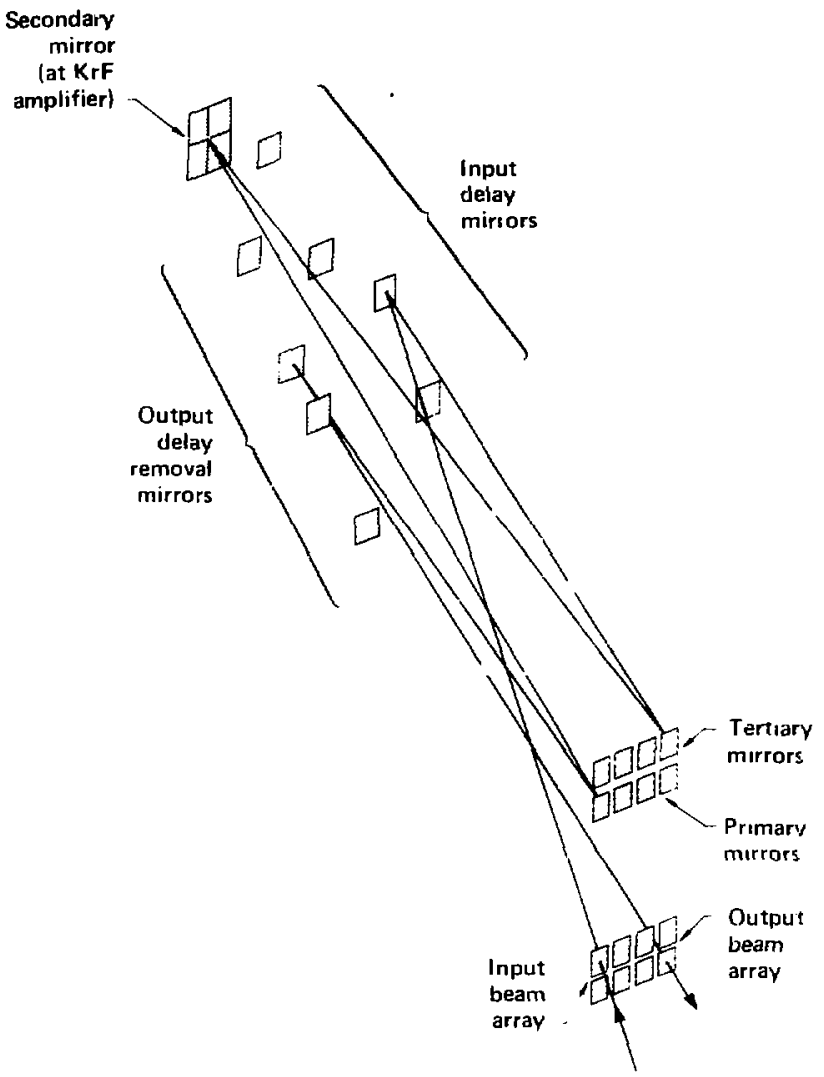

Fig. 7. Configuration of the optical train for a KrF pulse stacker, 
sequentially continuing this process down the train. All four mircors of each array are prealigned and fixed relative to each other, so the entire mount is aligned in one operation. The sensitivity and signal-to-noise ratios of this system are adequate with commerclally availabie components.

The mirro: that were chosen are dielectric coated, have aluminum substrates, and are water-cooled. The nominal fluence 1 inits imposed on the mirrors were $5 \mathrm{~J} / \mathrm{cm}^{2}$ at $24 \mathrm{~B}$ im and $100 \mathrm{~ns}$, and $4 \mathrm{~J} / \mathrm{cm}^{2}$ for the stokes pulse at $258 \mathrm{~nm}$ and $20 \mathrm{~ns}$; however, the final design has $5.9 \mathrm{~J} / \mathrm{cm}^{2}$ on one of the KrF stacker mirrors. The $10^{7}$ shot lifetime required for multilayer dielectric reflectors at this fluence is orders of magnitude beyond the curren: state of the art. At $248 \mathrm{~nm}$ and $20 \mathrm{~ns}$, the best commercial coatings have survived $1 \mathrm{c}^{2}$ shots at $2 \mathrm{~J} / \mathrm{cm}^{2}$ and single shots at $10 \mathrm{~J} / \mathrm{cm}^{2}$. clearly, this is an area requiring technology development.

The $K r F$ amplifier windows are constructed of panes of fused silica, each with a convex curvature to withstand the transient pressure differences; the thickness of each pane is $1 \mathrm{Lmited}$ to $10 \mathrm{~cm}$ by the temperature profile across the thickness. We also investigated aero windows of argon gas as an alternative that might eliminate the need for high-fluence solid windows; however, the gas used for a flowing curtain cannot contain fluorine because of the large absorption coefficient of the latter gas. Our calculations showed that flowing curtains of pure argon in contact with the laser gas resulted in such high mixing rates that the costs of recovering fluorine would be inacceptable. However, it may be possible to design more complex flowing-curtain configurations that could satisfy technical requirements in a cost-effective manner. Because of the large costs associated witt solid windows, further studies on gas-curtain windows are warranted.

\section{Key Technology Development Issues}

Perhaps the riast important result of the study is the identification of areas reguiring significant or early technological development. The following is an abbreviated list of the most important technology development issues identified in this study.

o Development of an output switch for a pulse-forming line that would have a shot life of $\geqslant 10^{7}$ shots uncer operating conditions of 2.7 MV, $0.3 \mathrm{TW}$, and $2 \mathrm{~Hz}$. 
- Development of effective moduläized diodes and control of insulator breakdown.

- Definition by experimental mathods of minimum magnetic-guide field requi rements.

- Development of dielectric catings for both mirrors and windows that, orstrating in the ultraviolet wavelengths, would be capable of withstanding $10^{7}$ shots in the 4 to $6 \mathrm{~J} / \mathrm{cm}^{2}$ range.

- Development of credible designs for fused-silica windows capable of surviving e-beam and pressure loadings, or civelopment of aero windows with appropriate control over mass flow and composition. Determiation of tie need for hel:um in beam tubes vs adequacy of dry $n 1$ trogen or air.

0 lefinition of requred optical homogeneity of KrF amplifier medium and seam-condition:ing gas and of minimum alignment tolerances required to adequately fill the Raman cell.

- Defin:tion of shielding requirements for both $x$ rays and noise.

c Determination of the effects of employing long-pump-pulse technology by varying amplifier sizing and stacker configuration to sptimize around less-expensive PFN technology.

The cost of the development time and effort represented in the above 1 ist is significant, but the technology is still young; it is highly probable that important innovations 1 ie ahead that can greatly reduce the technological $r$ isk, the required developmont time, and the eventual capital cost.

7. Summary of Laser-System "ost and Efficiency

In conclusion, we observe that the overall efficiency of the laser syster (38) was somewhat lesi tnan anticipated. An accounting of the primary energy losses is given in Table 3. It is possible that the e-beam deposition efficiency could be raised (from 578 to, perhaps, 658), but it is difficult to see where any great improvements could be made. The losses due to amplifier fill factors are already optimistic. However, the losses associated with wincows and mirrors can be improved, in principle, if efficient, high-damage-limit dielectric coatings can be developed. The intrinsic efficiency of $\mathrm{Kr} F$ at 
Table 3. Laser-sy'stem output and efficiency

$$
\text { (400-ns pulsed power). }
$$

\section{Laser electrical efficiency}

Source

Efficiency

Power (MN)

Wa].1-plug input

1008

78.6

E-beam deposition

578

44.8

Intrinsic KrF medium

108

4.48

Windows ard fil] factors

908

4.03

Mirrors

828

3.28

Raman conversion

80\%

2.63

Total lascr output

3.38

$2.63 \mathrm{kN}$

(1.3 MJ/shot)

Ancillary equipment losses

E-beam wall plug

$78.6 \mathrm{MN}$

gas-flow blowers

$5.6 \mathrm{MH}$

Magnetic guide fields

$2.8 \mathrm{MH}$

cooling water pumps

$0.7 \mathrm{MW}$

Total system input power

87.7 MN

Overall system efficiency $\frac{2.63 \mathrm{MN}}{87.7 \mathrm{MN}}=3.08$ 
40: ns probably cannot be bettered, and may be somewhat lower. The steady-state porer losses due to gas and water circulation and cooling ana to magnetic field establishment are 108 effects. Pinally, although a pure stacker m.ight save the 80 efficiency factor allosated to kanan convezsion, we do not $k$ now if the reduced $t$ am quality and prepulse isolation inpiled sy a pure stacke: would provide accepiable target performance.

The costs are sumarized in Table 4. They correspond to $\$ 2.67 /$ stored joule, which is consistent with previous estimates. It is interesting to note that the custs of the three major subsystems loptical, electrical, and building/mechailical) have total costs that are within 10 of one another, thus implying that there is no single area that could have significant leverage in reducing costs. We must seek to reduce costs by $f$ inding improvements in all a'cas--o: seek a major inrovation in the conceptual architecture.

Finally, we mention an inconsistency in the desıgn philosophy that should be remedied in future work. He intended the laser sysiem for use in an ETF, for which a lifetime of $10^{7}$ ̈hots is adequate. Mowever, there is a traicoff between economy and efficier ? thut. received little attention. The cost per unit of output energy is prouably tho best figure of merit for an ETF system. but we did not have the time or funds to explore some alternativ s that might reduce this cost $\{\$ 250 / \mathrm{J}$ output in a 20 -ns pulse in the ultraviolet $\}$. For example, welded-steel biamlines are probably not needed if nitrogen could be used instead of helium, separate delay mirrors for the input and output st.ackers are probably redundant, and facilities savirgs may be possible by including separate Raman cells in each heamline. In a brief, broadbrush study of this kind, we were not able to arrive at a point design of a recommended laser system. Instead, the greatest benefit of the study lies in both the technology issues identified and the alternative solutions proposed to attain a truly attractive short-wavelength laser driver. 

Table 4. Stacker-compressor laser syster costs (400-ns pulsed power).

E-beam generator systems

Optical systers

Buildings and ancillary equipment

Front end (148 of above)
$98.7 \mathrm{MS}$

$95.9 \mathrm{MS}$

87.2 MS

$39.4 \mathrm{MS}$
Total field

Home office

Total

Contingency (25\%)
$321 \mathrm{kS}$

$30 \mathrm{ks}$

351 MS

$88 \mathrm{MS}$

Construction cost (4th qtr 1979)

439 MS 
8. References

1. J. J. Ewing, R. A. Haas, J. C. Swingle, E. V. George, and W. F. Rrupke, IEEE J. Quant. Elect., QE-15, 368 (1979).

2. J. R. Murray, J. Goldhar, D. Eimerl, and A. Szoke, IEEE J. Quant. Elect., QE-15, 342 (1979). 
III. Technfcal Sumary: Appendices 
Appendix 1. E-Beams and Pulsed-Power Conditioning

J. Benford, Y. G. Chen, D. Dakin, G. Frazier,

T. Naff, and T. S. T. Young

Physics International company 
Section

Page

Figures

1-ii i

Tables

1-iii

1.1 Pulsed-Power Options

1-1

1.2 System Designs

$1-3$

1.2.1 Pulse-Forming Line System

$1-3$

1.2.2 Pulse-Forming Network System

1-10

1.2.3 Diode and Magnetic Field Coils

1-13

1.2.3.1 General Diode Parameters

1-13

1.2.3.2 Beam Deposition

$1-16$

1.2.3.3 Magnetic Field Coils

$1-17$

1.2.3.4 Diode Modularization

$1-1 \mathrm{a}$

$1-20$

1.3 Cost Structure

$1-22$

1.4 Technology-Development Issues

$1-24$

1.5 Conclusions

$1-25$

1.6 References

Figures

Figure

Page

1.1-1 Basic elements of the two pulsed-power

1-2

systems considered.

1.2-1 PFL module configuration.

$1-4$

1.2-2 PFL system efficiencies.

$1-5$

1.2-3 Pulse-forming line components.

$1-8$

1.2-4 PFL tube and diode conceptual design.

$1-9$

1.2-5 PFW module configuration.

1-11

1.2-6 Arrangement of two Marx modules

$1-12$

for PFN system.

1.2-7 PFW tube and conceptual design.

$1-14$

1.2-8 Constraints on diode modularization.

$1-19$

Tables

Table

Page

1.2-1 Marx generators for PFL driver.

1-6

1.3-1 KrF laser pulsed-power systems cost

$1-21$

breakdown (M\$) (14 amplifiers). 
Appendix 1. E-Beams and Pulsed-Power Conditioning

\subsection{Pulsed-Power Options}

The KrF amplifiers are driven from two sides by electron beams (e-beams) . which deposit their energy in the gas. This section describes the e-beam apparatus, including the diodes that generate the beams and the magnetic-field system that controls them.

The pulse duration chosen for the KrF amplifiers is an especially important parameter for the pulsed power system. In the range of a few hundred nanoseconds to $1 . \mu \mathrm{s}$, both the lumped-component pulse-forming network (PFN) and the distributed-component pulse-forming lite (PFL) systems must be considered (Fig. 1.1-1). The $\mathrm{KrF}$ driver requirements are near the upper bound of the PFL for practical length considerations and near the lower bourd of the PFN because of the many elements that are required in parallel to give the currents within the short pulse duration. Circuits intermediate between the two are also possible but are not considered here, since they are not as well developed technically. For pulse durations less than $0.5 \mu \mathrm{s}$, the PFL approach is probably preferable despite the fact that PFLs require the energy to be stored twice, once in the Marx generator and again in the liquid pulse-forming line, and in spite of the requirement for an output switch that must pass all the energy in the system at high voltage.

PFNs obviate the second pulse-charged energy store and have no output switch because the Marx is an element of the network. The PFN approach is made increasingly diffi:ult as the pulse duration is shortened. The number of elements in parallel reguired to keep the total system inductance low enough to supply the short pulse duration increases inversely with the square of the pulse duration. As the number of separate Marxes increases, the inductance of connections between them takes up a larger proportion of the allowed total; thus, for very short pulse durations, no selution is possible. Preliminary designs for an e-beam dri $r$ at 400 ns indicated that no PFN system could be built practically.

From the above considerations, it was decided that two systems would be designed: a PFL system to produce a 400-ns pulse for the amplifier, and a 
Pulse-forming line system

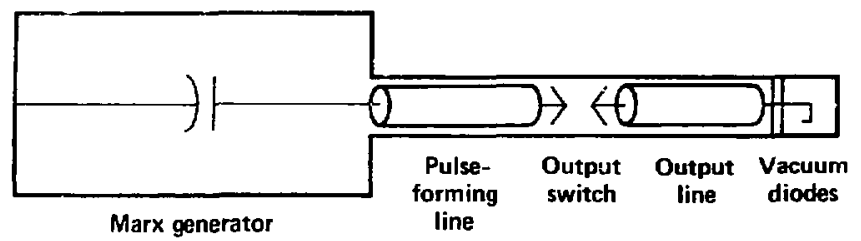

Pulse-forming network system

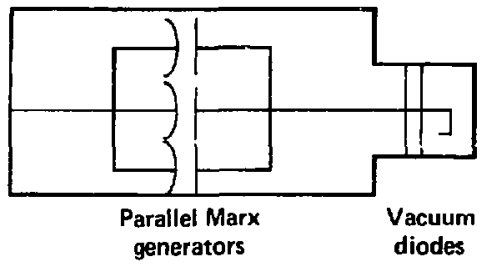

Fig. 1.1-1. Basic elements of the two pulsed-power systems considered. 
900-ns PFN system. Thus, each of the two system types would be operating in a pulse-duraticn regime appropriate to their best application. In the next section, we discuss both of these systems.

\subsection{System Designs}

\subsubsection{Pulse-Forming Line System}

The geometry c.f the pulse-forming line system is shown in Fig. 1.2-1. The energy is first stored in eight oil-immersed Marx generators, which, upon erection, charge eight water-dielectric pulse-forming lines on a time scale of about $2 \mathrm{js}$. The coaxial pulse-forming lines are in a 2 x 4 array. Each is discharged into an output line through an independent gas switch. The power then converges in two dimensions into eight circular tubes, which feed 16 separate riodes. The losses in this sequence of evonts are shown $1 n$ Fig. 1.2-2. The major losses are ir charging the pulse-forming line from the Marx and in passing the beam erom the ciode into the gas. In the first process, the losses are primarily in resistance of the capacitors and tne water iielectric; thus, the losses ultimately reside in rirculating flulds. similarly, the energy lost in switching the pulse-forming line to the output line resides in the gas flowing through the switch. The other majo: loss is to the hibachi and foil and the sidescatter and backscatter of electrons once they enter the gas chamter. The overall system efficiency is calculated to be 578; therefore, each Marx must store 1.3 MJ. Marx specifications are shown in Tabie 1.2-1. The basic stor age capacitor is $1.28 \mu \mathrm{F}, 50 \mathrm{kV}$.

The system is designed to operate for $10^{7}$ shots with only routine mairtenance required in this isterval. The capacitors in the Marx are derated by a factor of 2 in voltayje ( 4 in volume). Present-day high-stress capacitors just coming on the market have a life of about $2 \times 10^{5}$ shots. Lifetime is proportional to $v^{6}$ in this regime; therefore, decating by 2 gives a $2^{6}$ times $2 \times 10^{5}=1.3 \times 10^{7}$ shot lifetime. The Marx switches will erode about $100 \mathrm{~g}$ per switch in $10^{7}$ shots at an erosion rate of $50 \mu \mathrm{g} / \mathrm{C}$. This results in some widening of the gap $(\sim \mathrm{cm})$, which will be rompensated for by changing the gap pressure curing the lifetime of the system. The debris will be carried away by flowing the dielectric gas.

An alternative to Marx charging of the PFL is the double resonance transformer technique, whereby a low-voltage capacitor bank charges the 


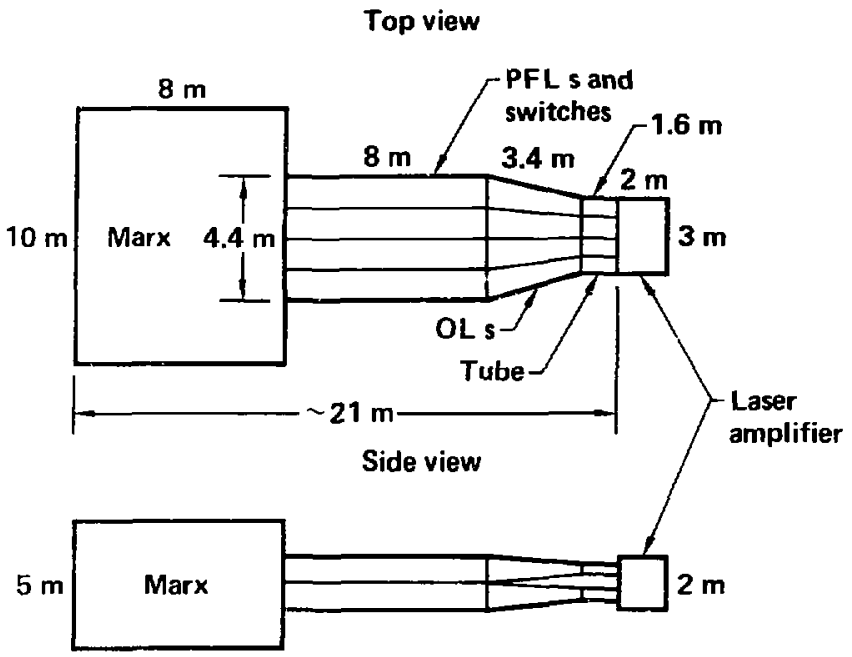

Fig. 1.2-1. PEL module configuration. 


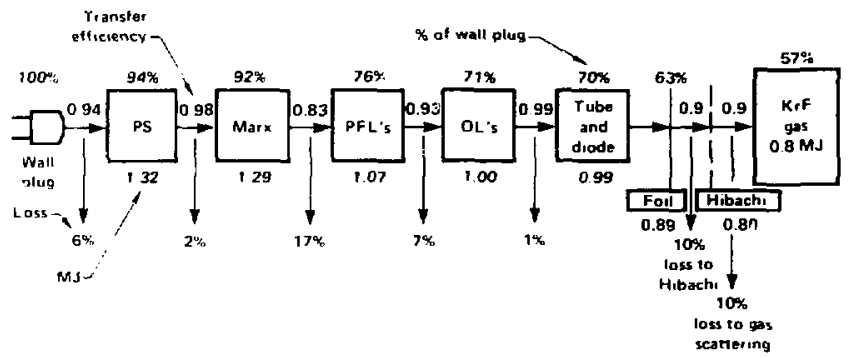

Fig. 1.2-2. PFL system efficiencies. 
Table 1.2-1. Marx generators for PFL driver.

\begin{tabular}{lccc} 
& $\begin{array}{c}\text { Single } \\
\text { Marx }\end{array}$ & Module & $\begin{array}{l}\text { Full } \\
\text { system }\end{array}$ \\
\cline { 2 - 4 } No. of Marx & 1 & 8 & 224 \\
Erected capacitance & $38.9 \mathrm{nF}$ & $310 \mathrm{nF}$ & $8.7 \mathrm{mF}$ \\
Stored charge & $0.12 \mathrm{C}$ & $0.93 \mathrm{C}$ & $26.1 \mathrm{C}$ \\
Stored energy & $160 \mathrm{~kJ}$ & $1.3 \mathrm{MJ}$ & $36 \mathrm{MJ}$ \\
Peak discharge current & $84 \mathrm{kA}$ & $675 \mathrm{kA}$ & $18.9 \mathrm{MA}$ \\
Charge transfer per switch & $\sim 0.2 \mathrm{C}$ & $5 a m e$ & same \\
Series inductance & $21.8 \mu \mathrm{H}$ & $2.73 \mu \mathrm{H}$ & $\mathrm{NA}$ \\
No. of stages & 28 & 224 & 6,272 \\
No. of capacitors & 112 & 896 & 25,088
\end{tabular}


pulse-forming line thrcugh a transformer. This system has the advantage of being inherently repetitive and eliminating the special connections and systems of the Marx technique. In the United States, Sandia Laboratories has developed this technology to the range of $\sim 1 \mathrm{MV}$ output voltage at $0.5 \mathrm{~kJ}$ with. high efficiency. Comparable values have been achieved in the Soviet Union. In both cases, the charge time of the PFL is 5 to $20 \mu 5$. For RrF, one needs $2.7 \mathrm{MV}, 10$ to $100 \mathrm{~kJ}$ with high efficiency. Using present technology, about 1000 trar.sformers would be needed. The use of larger transformers in smal!er numbers would squire a longer charge time due to the rather stringent incuctance/insulation requirements. This is impractical for wate: dielectric, necirise the resistive losses in the water would soon make the system inficicient. Therefore, extansive development would be nreded to use this tecinique for KrF. Consequently, we have projected the use of Marx-charged PFL for this resign study.

The diameter of the water-dielectric pulse-forming line is chosen on the basis of the statistics of water breakdown. (Water breakdown is arearieprndent since it is a statistical phenomenon.) We desizned for a 908 conntadence of having no more thar, one breakdown in $10^{7}$ shrits for each 1 ins. From the data of Sojka, this requirement translates to a safety factor of i.6: $=F_{\text {break }} F$, where $F_{\text {break }}$ is the Lupton ${ }^{2}$ breakdown field and $F$ is the sperating field at peak voitage of the line. This criterion gives a tield of epproximatnly $75 \mathrm{kV} / \mathrm{cm}$. The working field in this desizn was taken to be $60 \mathrm{p.V}: \mathrm{cm}$ to a!low an additioral safety factor for unknown effects associatec with operation with multiple pulses. With 10 additional time for the rise and fall of the pulse, the double-transit time of the pri, was made $440 \mathrm{~ns}$ (Fig. 1.2-3).

The output switch for each PFL is gas insulateci and crmmand triggerec. Each switch operates through six breakdown channels carrying $53 \mathrm{kA}$ each. This swirin can elther be one latge switch or three to four multistage columns. ${ }^{3}$ Losses in the switch are about $2 \mathrm{~kJ}$ per pulse.

From the output water line, the power converges into eight circular tubes; ajch tube Eeeds two diodes. The diodes are arranged in a $2 \times 8$ array. The ross-section to each tube (Fig. $1.2-4$ ) is dictated by the $2-x$ 3-m laser Interface and is, therefore, $100 \times 75 \mathrm{~cm}$. We have taken as an operating citorion that the vacuum insulator should flash no more than 1 shot in $10^{6}$. Assuming that three out of the many series invulator sections must fai before overvoltage makes it probable that the remainder fail, one 


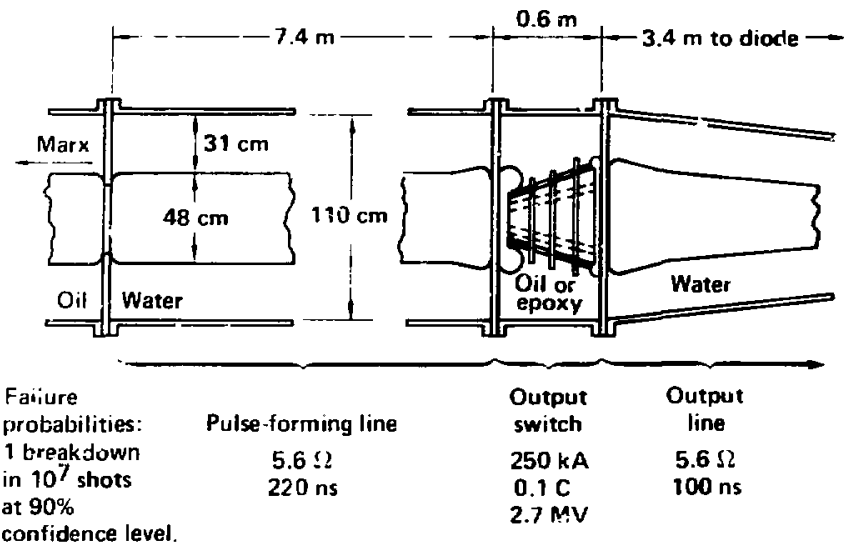

Fig. 1.2-3, pulse-forming line components. 


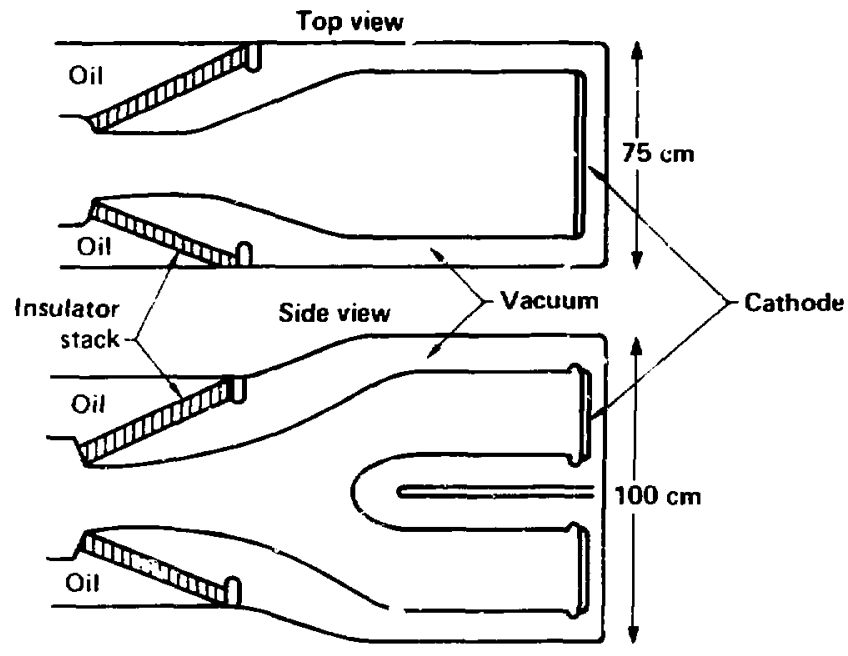

Fig. 1.2-4. PFL tube and diode conciptual design. 
insulator can be allowed to fail on average in every 100 shots. From this requirement, it becomes clear that a radial insulator configuration is not possible and that, in fact, the insulator must be a tapered stacked insulator. This approach is more expensive than the normal insaiator geometry. The cathode surface in vacuum is prevented from emitting to the walls by both the self-field and the externally applied field. Therefore, the entire tube is magnetically insulated. In the vacuum, the cathode splits (Fig. 1.2-4) into two lines; these rectangular lines carry the energy into the diode region. The length of the separate lines is chosen to ens're even flow of the electromagnetic energy.

The fields in water on the outside of the gradient rings are a concern, since they probably exceed $200 \mathrm{kV} / \mathrm{cm}$. They might bo buried in the plastic or, alternatively, a plastic shroud may be fitted over the whole tube and filled with water or weak electrolyte, eliminating the enhancement of the radial field. This liquid could also be used to grade the stack.

\subsubsection{Pulse-Forming Network System}

For the longer pulse duration of $900 \mathrm{~ns}$, a system employing a type-c pulse-forming network has been designed. It consists of six independent Marx generator modules, each composed of three parallel-connected Marxes. An oil transmission line connects each Marx/PFN to a tube (there are six tubes), and the overall energy transfer efficiency of this system is about $60 \%$.

We have chosen a type- $C$ network with branches formed of Marx generators. A two-section network is employed; it includes the fundamental and third harmonic of the main pulse. The number of branches necessary is determined by the required quality of the output pulse; we have specified a \pm 108 unifornity flatness during the pulse, with a rise of 108 of the pulse duration and a fall of 208 of pulse duration. Use of an extra section of the network would give 58 iniformity but was not considered to be a cost-effective approach. Two primary Marxes $(-90 \mathrm{~kJ}$ each) are used for the fundamental frequency, and one smaller Marx $(\sim 60 \mathrm{~kJ})$ is used for the harmonic. The PFN module configuration is shown in Fig. 1.2-5, and two Marx modules are shown in Fig. 1.2-6. The Marx layout is a slight extrapolation of contemporary designs. In the entire system, the number of switches is 14,000 and the number of capacitors is approximately $56,000\{50 \mathrm{nF}, 50 \mathrm{kV}$ ). Both switches and capacitors are derated in the same manner as for the PFL system. 
Side

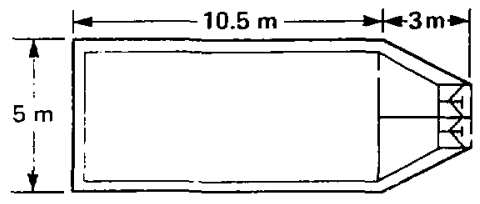

Front

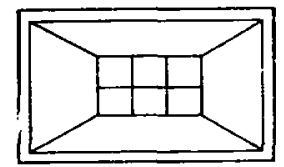

Top

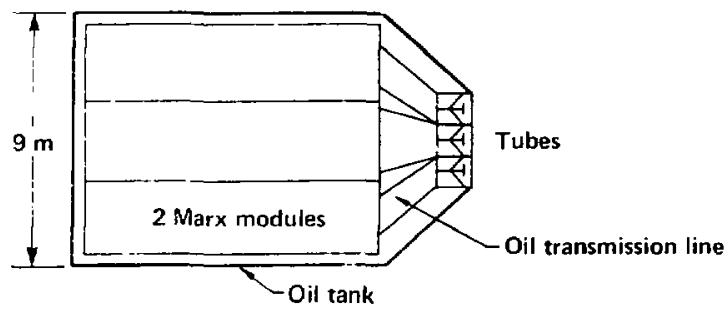

Fig. 1.2-5. PFN module configuration. 

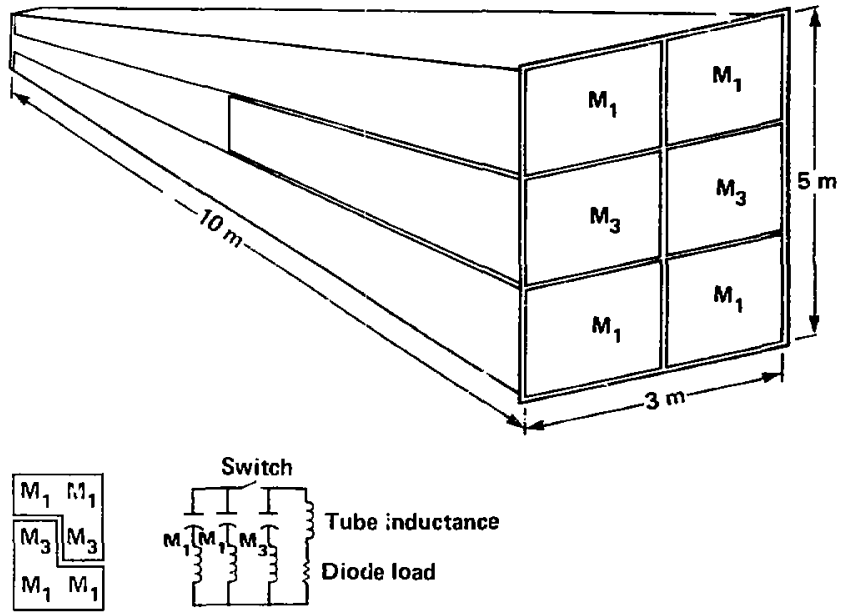

Fig. 1.2-6. Arrangement of two Marx modules for PFN system. 
The energy-transfer efficiency is determined by losses in transferring energy to the Marx (95\% efficient), from Marx to tube (878), and hibachi and gas-scattering losses (80\%). In addition, the slow rise and fall of the waveform result in $85 \%$ efficiency due to short deposition in the gas by low-voltage electrons.

All the PFNs feeding one side of a laser module harig in one oil tank, parallel electrically and physically. Current-carrying ground conductors must pass between them to make low-inductance connections to the individual oil Iines which connect to the tures. The six oil lines converge onto six tubes very similar in design to the PFL tube described above (Fig. 1.2-7). One difference is that only 6 diodes are used instead of the 16 in the PFL case. Fewer diodes are used with PFNs because the pinching of the beam in the cliodes is much less of a problem due to the lower current in each diode. As can be seen from the layout drawing, this configuration requires less volume anj is in some ways simpier than the PFL system.

\subsubsection{Diode and Magnetic Field Coils}

\subsubsection{General Diocie Parameters}

The emphasis of Physics International in this study contract was on the pulsed-power driver system. We determined magnetic field requirements and matched diode and pulsed power modules. It is clear that the diode itself must be mc'ularized to eliminate beam pinching. Magnetic fields can be ustd to reduce beam pinching and to provide for efflcient energy deposition in the gas. Experimental data ${ }^{4}$ at Lawrence Livermore National Laboratory (LLNL) indicate that modularization alone can reduce electron beam angles. Analyses ${ }^{4}$ were conducted at LLNL that scaled beam electron angles with the lateral dimensions of the beam and the anode-cathode spacing. The adequacy of diode modularization alone (no external magnetic fields) for the present bean parameters is doubtful for two reasons. First, in the study it was agreed to assume as a goal $90 \%$ beam transmission though the hibachi. It should be noted that present experimental values for hibachi transmission with magnetic fields are between 65 and 808 . This high transmission requires very low electron angles $\left(\leq 10^{\circ}\right)$, which are difficult to achieve without external fields. secondly, reduction of pinching by use of the radial electric fields caused by bringing the anode walls of the diode segments close to the cathode invariably 


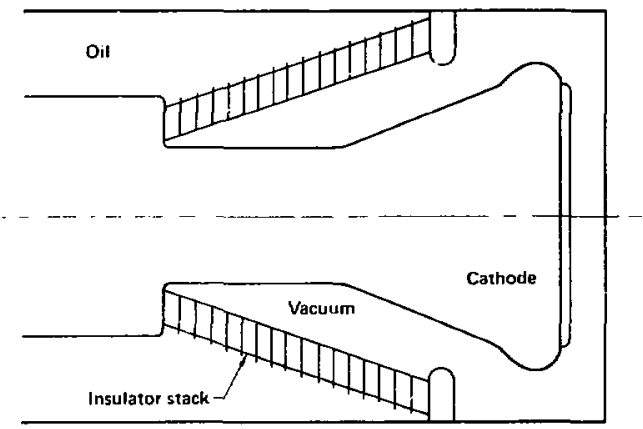

Fig. 1.2-7. PFN tube and conceptual design. 
causes emission from the sides of the cathode. Magnetically insulated flow parameters scale differently with voltage, spacing, and current than for the Child Langmuir diode. Thus, for the present beam parameters, experiments are needed to verify the required efficient transmission through the hibachi with diode modularization alone. In this conceptual design, we therefore use external magnetic fields as well as diode modularization.

It is important to minimize the magnitude of the external magnetic field in order to reduce the energy in the fields and maintain high overall system efficiency. The strength of the magnetic field is determined by constraints on efficient energy deposition in the gas as well as by beam electron angles in the diode.

Magnetic field requirements, diode modularization, and beam deposition are to a large degree independent of details of the diode design. Specifics, such as the type of cathode surface, design of the hibachi structure, foil materials, and cooling, are more appropriate for a point design effort and were not considered in detail in this conceptual study. Some comnents, however, might be made. Data on long-life field emission cathodes are minimal. However, both the uniformity and the lifetime of surfaces such as blades, carbon felt, plasma surface flashover, etched metals, and other cathode materials are under active investigation in the community. It is probable that reliable field emission cathode materiais will be found with lifetimes in excess of $10^{6}$ shots; these materials might be appropriate for near-term requirements. Two other diode alternatives exist. The first is the plasma-filled diode also under investigation for several applications. At the low repetition rates required for ICF, recovery time in these diodes should not be a limiting factor. The major parameter is the present limit on current density of $\sim 10 \mathrm{~A} / \mathrm{cm}^{2}$. The second is the use of thermionic cathodes. The current densities required $\left(\sim 30 \mathrm{~A} / \mathrm{cm}^{2}\right)$ have been achieved experimentally. LLNL $^{4}$ has suggested the possibility of grid electrodes in the diode to use the thermionic diode itself as the main output switch, but this possibility was not evaluated further in the conceptual study. One problem was identified for this approach, i.e., the insulator. Designs for low probability of insulator breakdown at pulse lengths of $\geq 1 \mu s$ are difficult with the limited space available, given the array of modular diodes. 


\subsubsection{Beam Deposition}

It has been shown from three-dimensional SANDYL code calculations that, without an external magnetic field, greater than 308 of the beam energY is scattered laterally out of the laser volume for the beam and laser parameters we are considerinc. With an external magnetic field, the beam loses energy laterally by diffusion. The beam energy-deposition profile transverse to the beam direction and outside the projected area of the beam is then approximately gaussian

$$
\exp \left[-\mathrm{x}^{2} / 4 r_{\perp}\right] \text {, with } r_{\perp}=10^{3}\left(\gamma^{2}-1\right)^{1 / 2} /|\mathrm{B}| \text {. }
$$

The strength of the magnetic field is then chosen so that the total area in the gauscian wings is less than 108 of the defined laser area $(200 \times 300 \mathrm{~cm})$. For $1 \mathrm{~atm}$, the required magnetic field is $0.65 \mathrm{kG}$ for $1-\mathrm{MV}$ and $0.9 \mathrm{kG}$ for 1.5 MV electrons. A $1-k G$ field was ther: selected to achieve the required 908 energy deposition, as defined at the onset of the study. It siould also be noted thist, when an external magnetic field is used to constrain the scattering of the bean, energy deposition along the beam can be calculated using one-dimensional Monte Carlo codes. For 1 atm and the given gas mixture, more than $95 \%$ of the beam energy is deposited in $200 \mathrm{~cm}$ at $1.3 \mathrm{MV}$. To provide maximum depasition uniformity along the beam, one would also increase the anode foil thickness to scatter the bean and thus increase deposition near tis: anode; this can be done with negligible energy loss in the foil.

The effect of sidescatterea beam electrons on the location of the optical mirrors at the ends of the laser cavity was also addressed in this study. To assess beam-energy deposition in the mirror, one needs both the incident flux $\left(\mathrm{J} / \mathrm{cm}^{2}\right)$ and voltage of the beam electrons. For those calculations, we chose the position of maximum energy deposition in the beam direction, which occurs approximately $60 \mathrm{~cm}$ from the anode. For a 1.3-MV beam, the main electron voltage at this location is $\sim 0.9 \mathrm{MV}$ for one beam and $\sim 0.6 \mathrm{MV}$ for the counterstreaming beam. Since the lateral scattering is a diffusive process, we assume an isotropic velocity distribution of beam electrons incident on the mirror. Assuming a fused-silica substrate with either $\mathrm{MgF}_{2}$ or $\mathrm{Al}_{2} \mathrm{O}_{3}$ coating, one can obtain the beam energy deposited in the mirror and conpute a temperature rise. With the mirror located 10 to $15 \mathrm{~cm}$ from the edge of the bear (as defined at the anode), the temperature rise is only a few degrees. 
Thus, with mirror separations of $20 \mathrm{~cm}$ or greater from the edge of the beam, it is very unlikely that any damage to the coatings or mirror substrates will cccur due to beam electrons.

\subsubsection{Magnetic Field Coils}

The axial magnetic field along the beam direction is produced by a pair of field coils in a Helmholtz geometry. The coils are rectangular $(5 \times 3.33 \mathrm{~m})$ and are separated by $5 \mathrm{~m}$. This geometry produces a $20 \%$ field uniformity in the lasing volume. The cross-section of the coils is $10 \times 10 \mathrm{~cm}$ and consists of 5 turns. For $1 \mathrm{kG}$ in the center of the laser volume, each coil stores $0.33 \mathrm{~mJ}$ of magnetic energy. This energy is transferred resonantly from a capacitor bank at $10 \mathrm{kV}$. Should $10 \%$ uniformity be required over the laser volume, the coil dimensions become $4 \times 7 \mathrm{~m}$ and are spaced $4 \mathrm{~m}$ apart. The corresponding energy in the field for $1 \mathrm{kG}$ in the center of the laser volume is $0.85 \mathrm{MJ} / \mathrm{kG}^{2}$ for each $\infty i 1$. Since the field energy for these coils is increased by $\sim 2.5$, the power-supply energy, power dissipation, and drop in system efficiency wuld scale by this factor.

The operating seguence has the bank firing about 2 ms before the e-beam system, so that the peak current in the coils is reached when the beams are injected. The full period of the oscillation is $8 \mathrm{~ms}$, at which time the energy has been returned minus small losses to the capacitor bank. At this time, interrupt switches are fired; the switches break the circuit until the next operating cycle. The interrupt switches are either vacuum-arc or liquid-metal plasma valves. The vacuum arc at present can intercupt a few kiloamps at $25 \mathrm{kV}$ and turn on and off in a microsecond; they have been shown to last for $10^{\mathrm{B}}$ shots. For this application, vacuum-arc switches must be incresed in current, or several must be used in parallel. present switches handle a few $k A$; the coils require $75 \mathrm{kA}$ peak.

The power system driving the field coil will dissipate approximate:y 4 MW during operations, causing a resistive energy loss of about $32 \mathrm{~kJ}$. Total losses in the system are estimated at about $15 \%$ of $0.33 \mathrm{MJ}$ per coil. These losses introduce an additional drop in system efficiency of approximately 48. 


\subsubsection{Diode Modularization}

The pulsed-power driver must be modularized to achieve efficiency (PFL) and feasibility (PFN). For maintenance, it may be advisable to make modules of the generator and diode match as much as possible. Therefore, constraints were determined for diode modularization and the number of diode segments selected. The pulsed-power system was then designed to match (one to one for PFN and one generator module for two diode segments, PFL). The number and size of the generator modules are not entirely unique, and some changes in the size or number of diode seyments could be accomodated and still match units.

In Fig. 1.2-8, we plot the self-field ( $\left.{ }_{5}\right)$ of the bean at the center of the long edge of the cashode as a function of the number of diode segments. The abscissa gives the number of modules in the horizortal (optical-axis) direction, and the two plots correspond to the number of segments along the flow direction. The total number of diode segments tnus considered ranges from 4 to 24 . The lateral spacing between the cathode and anode in each segment was constrained to be greater than an electron larmor radius (in the total field) to ensure magnetic insulation. The electron angle with respect to the anode plane is then given by $\theta=\tan ^{-1}\left[\mathrm{~B}_{\mathrm{S}} / \mathrm{B}_{\mathrm{z}}\right)$.

This scaling of electron angle has been verifiet both experimentally and in diode computer codes for a range of beam, diode, and magnetic field parameters. Also shown in Fig. $1.2-8$ is the self-field required to achieve $\theta=20 \mathrm{deg}$ for a 2-kG applied field (400-ns parametars) and for $1 \mathrm{kG}$ (900 ns).

We note that, even increasing the number of diode modules to 24 , one cannot achieve angles less than $15^{\circ}$ at the edge of the beam for applied fields $\leq 2 \mathrm{kG}(400 \mathrm{~ns})$. At the beginning of this conceptual study, it was agreed to define the required electron angle to be $20^{\circ}$; the number of diodes selected for the 400-ns beam parameters was therefore 16. Approximately the same electron angles result for $2 \mathrm{kG}$ using 6 diode segments for the $900-\mathrm{ns}$ case, since the voltage and energy were held constant and total current varied inversely with pulse length. As already stated, a field of $1 \mathrm{~kg}$ is needed for efficient energy deposition in the gas. The magnet power supplies were designed for a l-kG applied fielk. It may be possible to use $1 \mathrm{kG}$ in the diode for the 400-ns parameters by making use of the radial electric fields in the diode. Calculations of electron angle as a function of the ratio of applied and self-magnetic fields, which include finite geometry effects, 


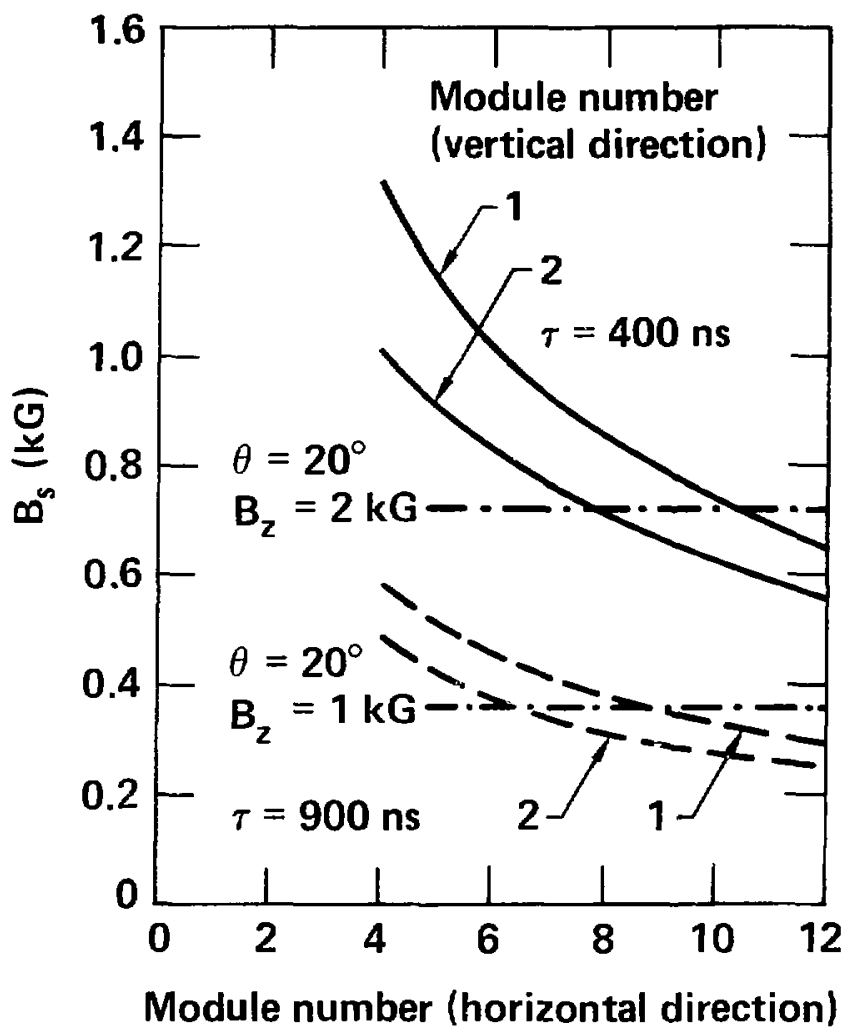

Fig. 1.2-8. Constraints on diode modularization. 
require diode computer codes and were outside the scope of this study. If the 1-kG applied field is inadequate for the 400-ns case, the overall system efficiency would have to be decreased either as a result of increased losses in the hibachi or increased losses in the magnetic field system. One obvious additional possibility is to use 2-kG fields and superconducting magnets, but the feasibility of superconducting coils with these dimensions was not investigated.

\subsection{Cost structure}

The costs of the $l i$ ne and network systems were separately estimated by breaking the system into its major cost factors. These factors are capacitors, switches, insulators (to include liquid insulators, such as oil and water, and solid insulators at the vacuum interface), metal (including oil handling and store and tank mobility system), electrical equipment (including controls, triggers, diagnostics, vacuum apparatus, water circulation, and the power system for charging), tube and diode, and the magnet system. Costs were estimated from current raw-material costs, coupled with estimates of the cost of fabrication, assembly, and testing.

Breakdown of the costs is showr in Table 1.3-1. It can be seen immediately that the capacitors and the means of switching capacitive energy constitute half the cost of the system. The five remaining elements are 108 of the total hardware costs. Fabrication of these hardware items requires abuut $20 \%$ of the hardware costs, and assembly and test another 108. Assembly and test of the more sophisticated PFL system would be approximately twice the cost of that for the PFN. It should be noted that these costs include spare parts: 108 of the capacitors, 108 of the switches, an oil transmission line for the network system, two spare water lines, and 108 of the vacuum insulators. The spare-part costs total $\$ 2$ million for the network and $\$ 2.5$ million for the line. Costs also include a small amount for handling and maintenance equipment.

The most striking feature of the cost estimates is their similarity. This, in part, is caused by the basic cost of the energy store, which is about half of the total. The real difference between the two systems, electrically speaking, is that the power is delivered at a level 2.5 times higher in the line system. This level is achieved by the addition of the pulse-forming line and the output switch. Although these components are high-technology items, 
Table 1.3-1. RrF laser pulsed-power systems cost breakdown (M\$) (1.4 amplifiers).

\begin{tabular}{|c|c|c|}
\hline & PFN $(900$ ns $)$ & PFL (400 ns) \\
\hline Capaci Lors & 19.6 & 20.6 \\
\hline Switches & 5 & 5.6 \\
\hline Insulator: & 4.6 & 5.8 \\
\hline Metal & 5.8 & 8.4 \\
\hline Electrical equipment & 8.4 & 10.8 \\
\hline Tube and diode & 4.4 & 4.6 \\
\hline Magnet system & 5.0 & 5.0 \\
\hline Hardware subtotal & 52.8 & 60.8 \\
\hline System fabrication & 10.6 & 12.1 \\
\hline Assembly and test & 5.3 & 6.1 \\
\hline Subtotal & 68.7 & 79 \\
\hline$G$ and $A$ fee & 17.2 & 19.7 \\
\hline Total & 85.9 & 98.7 \\
\hline Cost per joule stored & 2.65 & 2.72 \\
\hline
\end{tabular}


their basic cost is not high. The pulse-forming line is simply fabricated metal; the output switch, hosever, is rather sophisti ted. This estimate assumes that the switch has been fully developed and is mass procuced at the time of fabrication. The remaining elements, the tube, diode, and magnet system, are identical in the two cases with some allowance for differences in the assembly and testing costs. The two systems di fer by a toral of approximately 148, considering that constant energy store cost is about half of the total. The difference between the line and network systems is, therefore, appı sximately 258 of the remainder. The cost in terms of stored joules is about $\$ 2.7 \mathrm{C}$. This rather low cost is due primarily to mass production, a cost-redurtion $f$ actor that contemporary systems have yet to take advantage of.

\subsection{Technology-Developrent Issues}

The primary issue associated with the pulsed power for the KrF system is switching. The most difficult switch in the system is the output switch of the pulse-forming Iine. The switch must cariy substantial current at high voltage and do this repetitively upon a conimand trigger. The power level required is 0.33 Tw. Such a switch does not exist today. Single-shot very-high-power gas switches in the self-break mode are under development at present. One san anticipate substantial progress over the next few years. A repetit; ve switch at this level is not on the horizon, but there is no cuncamental reason why it cannot be developed. It appears that lower-power switches would be ceveloped for same military applications, but it is very likely that the specific switch needed for this system must be developed by the ICF program. It should be pointed out that the switshing requirement is held in common among several ICF drivers, most especially the light-ion approach being advanced by Sandia Laboratories. The major issues in the repetitive operation of such switches are the erosion of the ex. odes orer time and the deposition of debris on the insulator surface. The former probiem van probably be addressed by feeding new electrode material in as erosion occurs and the latter by vortex gas flow to carry the debris away.

The other switches in this system are less difficult to develop. The Marx switrh is a higher-power version of those available today. The lifetime requirement can be met by decreasing the gas pressure as the gap erodes, as described above. Therefore, development of this switch seems a 
straightforwari matter. The same can be said of the closing switches in the capacitor bank. However, the vacuum-arc opening switch must be developed. It is not clear that this switch will be developed for other applications, and the ICF program may have to do this job itself.

The remaining technology issues have to do with repetitive operation of insulators and diode componerts. At present, knowledge of the repetitive breakdowr sharacteristics of dielectrics is sketchy. For the system we've described to be viable, a study of repetitive breakdown of oil and water at the appropriate frequencies should be un ertaken. This will determine the safety lev: 's that can be employed in an enginuering facility. Similarly, a better tnderstanding of insulator brakdown unde. long-life repetitive conditions in vacuum :ould be required. Finally, the longtime behavior of catlode surfaces and anode foils under bonbardment by electrons is not well understood. We have designed a system on the basis of experience; further experimentation will be required.

The design we have described employs direct ext insions of contemporary pulsed-power technology: capacitive stores and gas closing switches. In the far term, commercial application will put a premium on improved energy density, i.e., compactness and high reliability. These rather basic considerations point toward the eventual introduction of inductive energy storage with, perhaps, rotating energy as the prime energy source. The key component in any magnetic energy-storage system is the opening switch, which must be capable of passing charging currents at minimum impedance and subsequently changing to high impedance to choke the current flow in a short timescale. The $\mathrm{L} d \mathrm{~d} / \mathrm{dt}$ voltage induced across ihe store inductor is used to drive the stored magnetic energy at high voltage into a parallel load circuit. The inductive-energy store offers about a $10^{3}$ increase in stored-energy density ovar capacitive-storage jystems, making these units much more compact.

A less-demanding technique is to use a rotating-store opening switch to pulse charge the PFL. For this scheme, switch pulse compressions of only 10 ( $50 \mu \mathrm{s}$ to $5 \mu \mathrm{s}$ ) are required. In fact, pulse charging with opening switches has already been done by Conte et al. 5 using single-shot $x$ ploding fuses. The remaining substantial obstacle is to make a repotitive switcin.

The rotating store could also be used for charging a pulse-forming Iine directly through a transformer if it could be made fast enougin. The problem at present is that the high-energy-density liquid dielectrics we now use 
(primarily wate:r) have resist: -e losses that would make the system very inefficient for charge tines greater than $10 \mu s$. If a 10- $\mu \mathrm{s}$ alternator were produced, fast charging of pulse-forming lines through a step-up transformer would make a very inexpensive, compact system. An alternative is to use solid plastic dielectrics, which do not have a resietive loss but which have lower energy density and, therefore, large volumes.

We conclude chat the pulsed-power system described here can be implementec by continual developmert. of contemporary systems. Fowever, great leverage toward truly commercial systems can be obtained by developing inductive storage and/or employing rotating machinery.

\section{5 Conclusions}

E-beam-driver conceptual system designs have been developed for both 400-ns (PFL) and 900-ns (PFN) long-pulse amplifier pulse durations. Both systems are extrapolations of contemporary technology. The PEN system entails less technology extrapolation; the technology developaents for the PFI systen, primarily the repetitive, high-power gas output switch, will probably have been developed by the time ETF enters the design phase.

The cost striciure of the two designs is quite similar when compared by element. The PFN system appears to be somewhat less expensive than the PFL. A countervailing consideration is that the short-pulse system operates in a more advantageous regime of laser kinetics. Therefore, the increased price and complexity may be worth the effort. The PFN system would be much more difficult to build at shorter pulse durating and is, therefore, restricted to the longer pulses. Construction of a PFL with Ionger pulse duration would not have substantial cost impact, but the longer pulse Juration would increase optics costs and cause poorer kinetic performance of the laser medium.

For commercialization, great leverage can be achleved by developing inductive-energy storage. This concept can result in a much more compact and inexpensive e-beam generator. 
1.6 References

1. R. Sojka, G. Simcox, and R. Shaw, Dielectric Performance under Repetitive Elecicrical Stresses," Physics International Company, San Leandro: Calif., PIFR-1297 (1979).

2. R. Elbert and $*$. H. Lupton, Extrapolation of AWRE Breakdown Dacā, Naval Research Laboratory Internal Report.

3. T. Naff (private communication).

4. L. G. Schlitt (private comunication).

5. D. Conte, R. Ford, พ. H. Lupton, J. D. Shipman, P. Turchi, and I. M. Vitkovitsky, Inductive Charging of Pulse Lines, Naval Research Laboratory, Memo Report 3742 (1978). 
Appendix 2. Optical Systems

M. R. Flannery, G. M. Perron, S. N. Suchard, and D. E. Vandenburg Hughes Aircraft Co.

L. G. Seppala

Lawrence Livermore National Laboratory 


\section{Contents}

Section

Page

Figures

2-ii i

Tables

$2-i i i$

2.1 Introduction

2-1

2.2 System Options

2-2

2.2.1 System Architecture

2-6

2.2.2 Mirror Construction

2-7

2.2.3 Mirror-Mounting Strategy

2-7

2.2.4 Mirror-Aligment Strategy

$2-8$

2.2.5 KrF Amplifier Windows

2-9

2.2.6 Beam-Path Enclosure

2-9

2.2.7 Beam-Path Gas

2-9

2.2.8 Raman-Cell Windows

$2-9$

2.3 Description of the Baseline Design

2-9

2.4 Cost scaling

2-13

2.5 Technology Development

2-15

2.6 Conclusions

$2-20$

Figures

Figure

Page

2.1-1 Top view of the compact unit-magnification $2-3$ four-beam stacker.

2.1-2 Top-front perspective view of the compact $2-4$ unit-magnification four-beam stacker.

2.1-3 Sensors and servos in the pulse stacker and the double-pass Raman cell.

2.3-1 Location of sensors in relatica to mirrors.

Tables

Table

2.4-1 Optical-system cost summary. 2-14

2.5-1 Optical-train component $2-18$ number and fluence-loading summary. 
$2-i v$ 


\subsection{Introduction}

The objective of the optical system is to deliver a high-quality 1.5-MJ, 20-ns light pulse to a fusion pellet with high efficlency, with no prepulse energy on the target, and with a smooth wave front for pointing and focus control. The KrF excimer laser operating at $248 \mathrm{~nm}$ has both the high efficiency and short waveiength required of an ideal fusion laser, but this laser suffers Erom $a$ very short storage time $(\sim 3 \mathrm{~ns})$. In the present application, this limitation is overcome by using pulse stacking. The power-amplifier module is fed by an optical-delay line that temporally stacks a sequence of $\mathbf{N}$ short pulses (pulse stacking) of pulse length $r$ through the amplifier at slightly different angles (angular multiplexing). By overfilling the amplifier with each pulse, it is possible to extract energy from the amplifying medium for a pulse length of $\mathrm{N} t$. This allows efficient punping of the amplifter by long-pulse electron beams. Angular multiplexing of the short pulses allows them to be raparated spatially at the output of the amplifier, where their relative aelays are removed in a second optical-delay line. The pulses are then reassembled into a spatial matrix of collimated beams that is directed toward the final-focusing optics. This report examines pulse stackers with $r=100$ ns and either $\mathrm{N}=4$ or $\mathrm{N}=9$.

Further pulse compression, beam cleanup, and prepulse isolation are accomplished by using the KrE laser as a pump for a double-pass backward Raman scattering seil filled with methane. This cell converts the 248-nm, 100-ns, 1.9-MJ KrF pump pulse of poor wave-front quality to a 268-nm, 20-ns, 1.5-MJ Raman-shifted pulse with an optical quality dependent on the quality of the stokes input beam and on effects of gas-density fluctuations in the Raman cell and beam tubes.

since the KrF pump beam is not aimed toward the target-focusing optics, the prepulse energy arising from scattering crosstalk between the stacked pump pulses dors not reach the target. The only prepulse energy that reaches the target is due to scattering of the pump beam in the Raman cell (on both passes) and scattering of che stokes beam as it is amplified in the first pass. Buth effects are small.

The stokes input to the cell requires only $1.5 \mathrm{~kJ}$ to generate the $1.5-\mathrm{MJ}$ nutput, and this input, in turn, is generated in a smaller Raman cell that 
reguires an input of only $1.6 \mathrm{~J}$. The general architecture of a four-beam stacker is show in Figs. 2.1-1 and 2.1-2. (A combination of 14 stackers pumping a common Raman cell is shown in Fig. 3.4-3.)

The optical system is supported by a beam-path conditioning system, a mirror-cooling system, and an alignment system. The beam-path conditioning system removes heat from both the beam-path gas and the Raman gas while maintaining a specified level of turbulence or laminar flow; the mirror-anoling system removes heat from the mirrors to control thermally induced disitortion in the large mirrors; and the alignment system--comprising sources, sensors, processing electronics, and servos--senses beam-train misalignmezts and corrects them by two-axis steering of the system mirrors. The aligrinent system takes advantage of the separation of the delay mirrors to steer individual beam lines at these mirrors, while the number of servos is reduced by combining the mirrors (either 4 or 9) at the pulse-stacier primary and tertiary mirrors into arrays and then steering all beams in one stacker at these locations. The location of image sensors, mirrors, mirror arrays, and steering servos is shown for one stacker and the Raman cell in Fig. 2.1-3.

\subsection{System Options}

The major options that were consldered in the study are given below. (The preferred systems are indicated by asterisks.)

o System architecture

$\infty$ Four-beam stacker with unit magnification in the stacker telescope.

$\infty \quad$ *Four-beam stacker with $2.45 x$ beam expansion in the stacker telescope.

$\infty$ Nine-beam stacker with unit magnification in the stacker telescope.

N Nine-beam stacker with $2.45 \mathrm{x}$ beam expansion in the stacker telescope.

- Mirror construction

$\infty$ Metal- and dielectr ic-overcoated glass mirrors.

$\infty$ *Dielectric-overcoated aluminum mirrors.

$\infty$ Dielectric-overcoated opper mirrors.

$\infty$ Dielectric-overcoated molybdenum mirrors.

$\infty$ Dielectric-overcoated beryllium mirrors. 


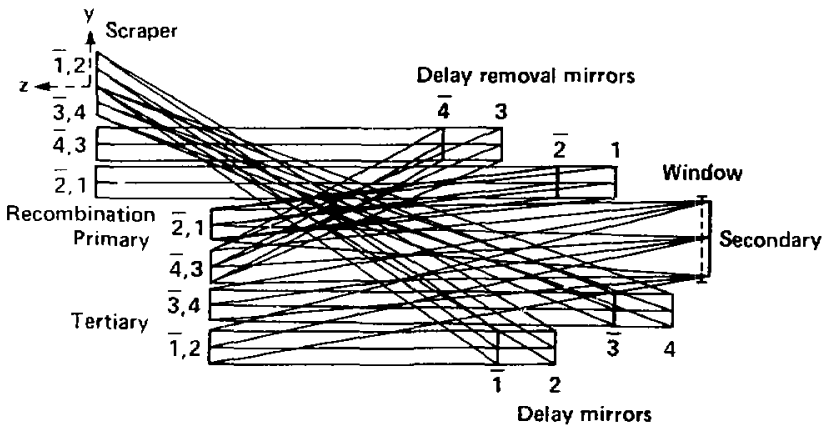

Fig. 2,1-1. Top view of the compact unit-magnification four-beam stacker. 


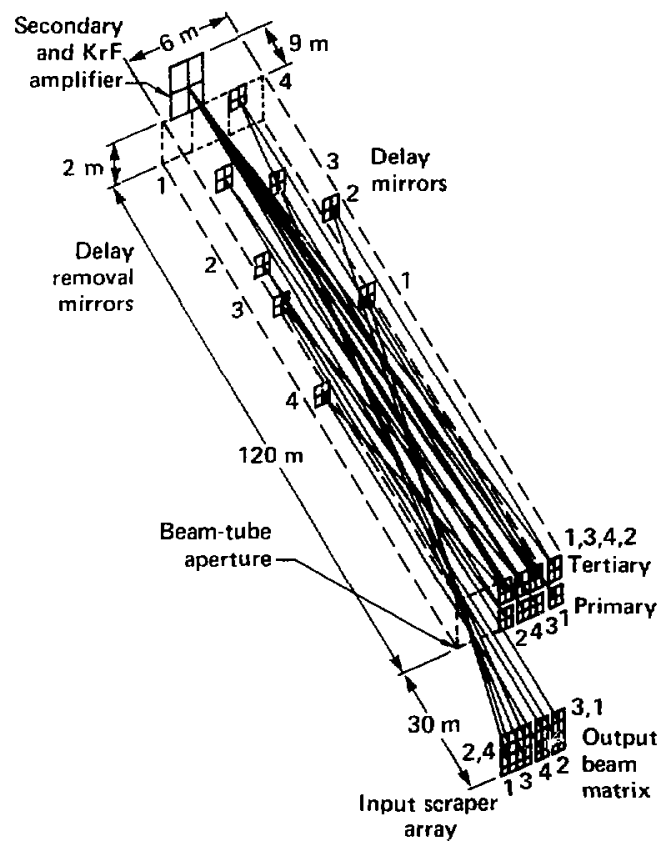

Fig. 2.1-2. Top-front perspective view of the compact unit-magnification four-beas. stacker. 


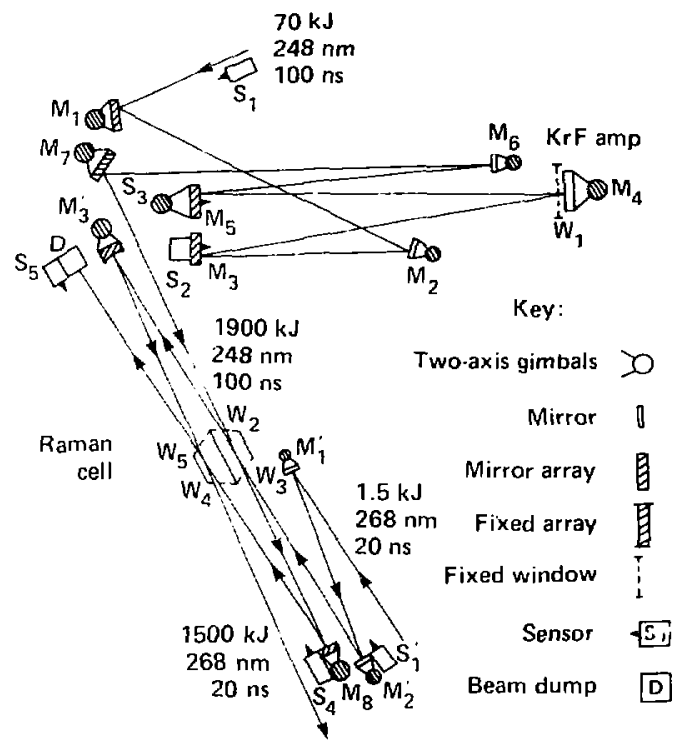

Fig. 2.1-3. Sensors and servos in the pulse stacker and the double-pass Raman cell. 
$\infty$ All mirrors on individual servo-controlled two-axis mounts.

$\infty \quad$ *Fixed, prealigned mirror arrays mounted on a common servo-controlled two-axis mount.

- Mirror-alignment strategy

D Central-beam-reference aligmment system.

m Inner-annulus-reference alignment system.

no Outer-annulus-reference alignment system.

- Image-alignment system.

- KrF amplifier windows

oo Thin single-pane, spherical-shell fused-silica windows separated from the amplifier by a long acoustic baffle.

() tThick faceted, spherical-shell fused-silica windows located 5 to $20 \mathrm{~cm}$ from the amplifier.

- Beam-path enclosures

$\infty$ Individual beam-path tubes in the pulse stacker.

oo *A single beam-containment house for each pulse stacker.

- Beam-path gas

$\infty$ Argon.

$\infty \quad$ *Helium.

- Raman-cell windows

$\infty$ Sheared-flow inert-gas windows for the Raman cells.

(IJncoated Brewster's-angle fused-silica windows for the Raman cells.

The justifications for choosing the indicated options are given in the following subsections.

\subsubsection{System Architecture}

The 4-beam stacker was chosen over the 9-beam stacker because the latter requires a delay line approximately $60 \mathrm{~m}$ longer than the former. Although the pulsed-power systems are comparable in cost, the cost trade-off is overwhelmingly dominated by the larger laser-containment building that would be re'uired by the 9-beam stacker.

From an optical standpoint, four-beam stackers have shorter pathlengths; require fewer and less-expensive alignment sensors; have fewer mirrors, mirror mounts, servo loops, mirror-cooling loops, and beam tubes; and require less spaceframe and less dielectric-coated mirror area. However, the four-beam 
stackers have larger mirrors, which are more difficult to polish, coat, and mount and which are much more sensitive to thermally induced distortion and to mounting stresses.

A stacker telescope with a net beam expansion ratio or $2.45 x$ was chosen. The unit-magnification stackers are insensitive to optical damage in the input delay line and require a smaller inventory of mirrors and mirrors mounts than stackers with net magnification. However, one can choose a net magnification that simultaneously reduces the area of the input mirrors and still protects the input opticis from damage. Savings are realized in mirror blanks, fabrication, and mounting because of the smaller mirror sizos.

\subsubsection{Mirror Construction}

Dielectric-overcoated aluminum mirrors appear to be the best choice. Beryllium was eliminated because of its tigh toxicity and cost. The high-power mirrors absorb up to $0.47 \mathrm{w} / \mathrm{cm}^{2}$, which requires modest water cooling. The high cost of low-expansion glass and the difficulty of [abricating a high-pressure, small-channel heat exchanger that would maintain the temperature rise of the coatings bejow $150^{\circ} \mathrm{C}$ have generally eliminated glass mirrors. Because the thermal requirements are so modest, aluminum is preferable to copper, which is more expensive, more dense, ano has essentially zero microyield stress. Since the hlgh rigidity of molybdenum will probably not he necessary to reduce thermal distortions and mounting distortions for mircors used in essentially fixed positions, its high cost, high density, and the difficulty of fabricating large, complicated substrates from sintered material eliminate molybdenum in favor of aluminum. The final mirror design that was selected is an investment-cast aluminum substrate dip brazed to a thick aluminum faceplate.

\subsubsection{Mirror-Mounting Strategy}

Since the laser system operates in thermal equilibrium, it appears possible to prealign the mirror arrays on c honeycomb-steel optical table and to maintain the array over long periods of time to within 5 to $10 \mu \mathrm{rad}$. Calculations show that such a prealigned optical table with mirrors on one side can be mounted vertically with maximum displacements of only $\pm 10 \mu \mathrm{rad}$. This arrangement would result in large savings in both the cost and complexity of the alignment system. 


\subsection{Mirror-Alignment strategy}

Cencral-beam-reference alignment systems use either a fraction of the main laser pulse or a co-propagating alignment pulse that is fired between the main laser pulses. These systems were rejectud because meta? mirror substrates were chosen for thermai and cost considerations. Therefore, access to the beam woul, require hole-cuupling mirrors to pass the alignment beam through the $\mathrm{KrF}$ dielectric reflector and then through small holes in the metal substrate. A central-beam reference pulse could be obtained by firing the initial oscillator at twice the repetition rate of the laser system. However, the metal mirrors would require a bonded glass layer (to cover the coupling holes) that would degrade the thermal value of the metal substrate.

An inner-annulus system would overcome most of these nbjections but would require hole.s in the centers of the mirrors; these holes would pruduce a rogion of untroped gain in the KrF amplifiers.

An outer-annulus system would be the system of shoice except that a large number of exp nrive sensors are needed to ga in a iarge field of view (Fov). This system has the advantage that it uses a.d outer annulus outside the nigh-power beam print on all mirrors except the secondary mirror behind the KrF amplifier. In the case of this mirror, if the alignnent beam were off the high-power beam print, there would be a region of untapped gain. To avosd this difficulty, special tilted annuli would have to be ground on the primary anci tertiary mirrors to insert and extract the reference beam from the high-power beam print or the secondary mirrur. These arinuli could be mad: either by diamond turning or by using hulographic gratings on the annuli of the primary and tertiary mirrors. In either case, rirror fabrication costs would increase.

The best alignment technique appears to be an image-alignment system that uses auxiliary light sources outside the high-power bean print. The high-power laser mirrors are aligned by tilting them to reflect light from the sources to predetermined locations inside an imaging sensor system that is locatec outside of the high-power laser beam print. Derated laser diodes, which are both reliable and i nexpensive, serve as bright-point light sources for a high-resolution inage. The cost of the image-cilignment system is a strong function both of the number of delay lines that can be multiplexed to each image sexisor and of the distance along each line tnat the sensor can mainrain resolution and collect enough energy to maintain a sufficient 
signal-to-noise ratio (S/N). The sensor requirements for the four-beam stackers are relatively modest: two sensors are required for cach compact stacker,

\subsubsection{KrF Amplifier Windows}

The energy required to flow gas through a long acoustic baffle and to recover the fluorine from it is prohibitive. Therefore, four-pane fused-silica windows located from 5 to $20 \mathrm{~cm}$ crom the amplifier rere chosen. The windows could be up to $10 \mathrm{~cm}$ thick with a long radius of curvature, as required to withstand the shock pressure generated by heat depositior in the amplifier medium.

\subsubsection{Beam-Path Enclosure}

The four-beam net-magnifiration stackers can be so compact that a single beam tube or beam house for each stacker is less expensive and complex than individual beam tubes.

\subsubsection{Beam-Path Gas}

To maintain an alignment resolution of $\pm 1 \mathrm{~mm}$ over a distance of $400 \mathrm{~m}$ for $\mathrm{sin}$ alignment beam at $820 \mathrm{~nm}$, a sensoi with 28 -cm-diam optics and an atmospher thermal structure constant of $\mathrm{c}_{\mathrm{T}}^{2}<0.05^{\circ} \mathrm{C}^{2}$ for argon and $\mathrm{c}_{\mathrm{T}}^{2}<$ $3.0^{\circ} \mathrm{C}^{2}$ for helium is required. Therefore helium is completely aciecuate, but argon might be marginal.

\subsubsection{Rar:an-Cell Windows}

Brewster's-angle windows were chosen. The energy to maintain a sheared-flow inert-gas window is prohibitive if the flow is fast enough to prevent the buoyancy differences between $\mathrm{CH}_{9}$ and argon or helium from producing a lens in the Raman cell. Brewster's-angle windows remuve the difficulty of requiring a high-damage-level antireflection coating at both $24 \mathrm{~B}$ and $266 \mathrm{~nm}$ but require the $\mathrm{KrF}$ optical train to preserve a linear polarization.

\subsection{Description of the Baseline Design}

This section describes the baseline design for a $2.45 \mathrm{X}$ magnification four-beam stacker and $i$ ts optical components, alignment system, Raman cell, and support structures. 
A 2.45X magnification four-beam stacker was used as a baseline and is similar in layout to the telsscope in Figs, 2.1-1 and 2.1-2. The three-spherical-mirror telescope expands each 33.7- $\times 33.7-c m$ collimated input beam to fill the 2- $x$ 2-m amplifier aperture and then reduces it back to a collimated output beam at $82.5 \times 82.5 \mathrm{~cm}$. The amplifier aperture is determined by both the pumping time and the kinetics of the gain medium, while the beam size in the output end of the stacker is determined by the $5-J / \mathrm{cm}^{2}$ fluence requirement for $\mathrm{KrF}$ in the Raman cell. A preliminary design assumed that the tertiary and primary mirrors were single spherical surfaces. Since the fluence levels on the output tertiary mirror become extreme if the beam

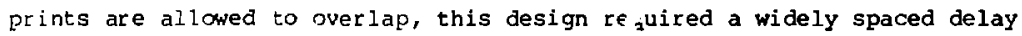
mirror array that occupied a prohibitively large volume.

By dividing tha primary and tertiary mirrors into segments, the volume occupied by the stacker can be greatly reduced. The individual mircor segments are tilted so that the delay arrays are more closely spaced. This stacker requires a beam tube $120 \mathrm{~m}$ in length with a cross section of only $2.1 \times 6.2 \mathrm{~m}$. This baseline design has angular optical distortions of less than $10 \mu$ rad in each beam. The image alignment system, with one stacker and the Raman cell, is shown in Fig. 2.1-3.

In i is simplest form, an alignment sensor consists of a detector array at the focus of a lens. This sensor is affected by its own angular misalignment, but this type of er for can be monitored if the sensor looks directly at a laser diode in the spaceframe. Individual mirrors in the pulse stacker can then be aligned by monitoring the positions of reflected images of fixed laser dioce sources in the space srame, using the image position of the directly viewed diode as a reference.

The sensors control the steering servos on the first mirror they see down the beam line by imaging the laser diode light sources located on che second mirror down the beam line. For example, sensor $S_{1}$ forms an image of the laser diodes on mirror $M_{2}$ and compares these point images with a perfect-alignment reference image to generate error signals that are used to drive the servo on $M_{1}$ until the images agree. When the images agree, the central ray of the $K r F$ laser beam, which has been aligned adjacent to $s_{1}$ by the front-end alignment system, will reflect off the centers of $M_{1}$ and $M_{2}$. Similarly, $s_{2}$ can align $M_{2}$ and $M_{4}$, providing handoff between alignment sensors. The sensor images follow a skew path down the optical train because the sensors are located adjacent to the mirrors, as shown in 
Fig. 2.3-1. Thus, the Fov eventually walks off the mirror (after about three reflections) even if the resolution or $S / N$ did not 1 imit the sensor.

placing the sensors adjacent to the mirrors rather than attempting some form of aperture sharing allows the sensor to be shared by adjacent beam lines and renuces the number of sensors. As shown in Fig. 2.3-1, one sensor can align four beam lines in a stacker when the sensor is located at the center of the primary or tertiary array, or one sensor can align two arrays if the sensor is located at the joints between the arrays on $\mathbf{M}_{8}, \mathbf{M}_{2}^{\prime}$, or $M_{3}^{\prime}$. To calculate the resolution of the sensor, assume that the sensor is a 512- $x$ 512-element array of 20- x 20- $\mu \mathrm{m}$ diodes with overall dimensions of $1.024 \times 1.024 \mathrm{~cm}$. The limiting FOV in the system will be the $87.5-\times 87.5-\mathrm{cm}$ delay mirror, located $120 \mathrm{~m}$ from the tertiary, that gives the sensor at the tertiary a 7.29-mrad Fov; this corresponds to a 1.97- $\times 1.97-m$ area in the object plant $270 \mathrm{~m}$ away at the scraper mirrors. We will assume the sensor has a zoom lens that keeps the object-plane area fixed at this value. The longest source-to-sensor distance is $290 \mathrm{~m}$, between $s_{4}$ and the closest $M_{6}$, while the shortest tistance is approximately $150 \mathrm{~m}$, between $\mathrm{s}_{1}$ and the closest $\mathrm{M}_{2}$. A sensor with an aperture dianeter (d) of $28 \mathrm{~cm}$ has a diffraction-limited resolution angle $2 \theta_{\text {dif }}=1.22 \lambda / d=3.57 \mu \mathrm{rad}$ (at $\lambda=$ $820 \mathrm{~nm}$ ), which corresponds to $1.04 \mathrm{~mm}$ in object space at $290 \mathrm{~m}$. If we assume there is one sensor element per resolution spot (i.e., the sensor array covers a $0.533-\times 0.533-m$ area in object space), then the magnification is $\mathrm{m}=$ -0.0192 , the focal length is $f=5.69 \mathrm{~m}$ at $290 \mathrm{~m}$ and $\mathrm{f}=2.94 \mathrm{~m}$ at $150 \mathrm{~m}$ for a zom ratio of about 2.0. A Questar 12 is a suitable foreoptic for this sensor: $f=4.572 \mathrm{~m}$, clear aperture $=30.48 \mathrm{~cm}$, FOV $=8.03 \mathrm{mrad}$, and obscuration ratio $=23.68$. The sensor can cover the whole FOV either by insezting some additional optics to match the 1.97- $x$ 1.97-m object plane to the sensor or by stepping the sensor pointing mirror across the object plane. The sensitivity of the sensor will be found assuming the sourco is a laser dinde with 28 of its power $P_{O}=5 \pi w$ at $\lambda=920 \mathrm{~nm}$ radiating into a cone with a half-apex angle of $\theta_{r}=52.4 \mathrm{mrad}$. If the high-power mirror has a ceflectivity of $R=0.7$ at $\lambda=820$ nm and the sensor has a transmission $T=$ 0.2 , a diameter $d=28 \mathrm{~cm}$, and an obscuration ratio $r_{0}=0.24$, the image-plane power is

$$
P=0.02 P_{0} R T\left(1-r_{0}\right) \frac{(\alpha / 2)^{2}}{\left(L \theta_{r}\right)^{2}}=9.03 \times 10^{-10} \mathrm{~W},
$$




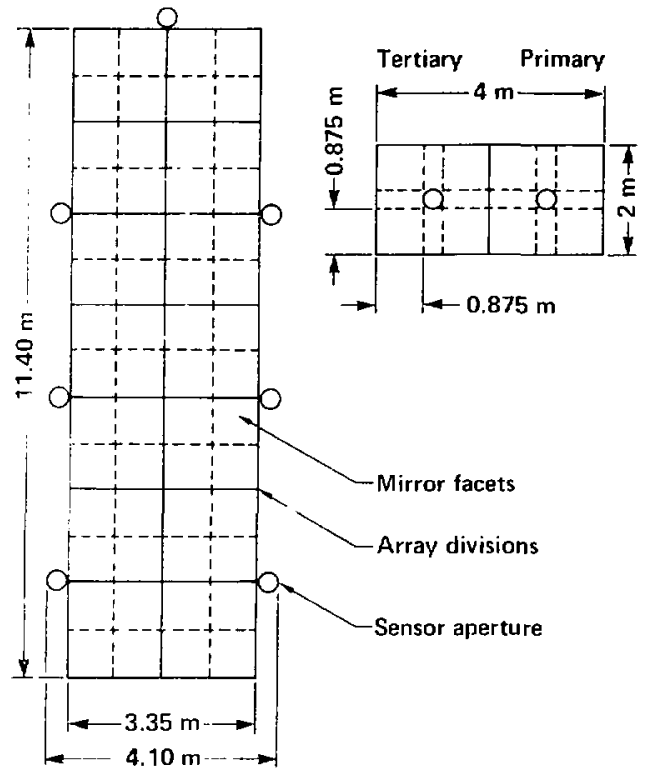

Fig. 2.3-1. Location of sensors in relation to mirrors. 
assuming the diode is the maximum distance ( $L=290 \mathrm{~m}$ ) away. Since this power could be spread by system aberrations over, perhaps, 9 sensor elemencs, the power falling on an element is about $10^{-10} \mathrm{~W}$. For a typical 20-ms integration time and the $20-\times 20-\mu \mathrm{m}$ diode area, the energY/area $=$ $0.75 \mu \mathrm{J} / \mathrm{cm}^{2}$, which is at the low end of the detectable energy density for diode or CCD arrays but which is still easily detectable.

\subsection{Cost scaling}

The costs for the four-beam stacker baseline design are divided into categories in Table 2.4-1. The greatest cost sensitivities are in the windows, where small changes in thickness mean large changes in cost, ano in areas of technological uncertainty, such as polishing and, particularly, coating.

The approsimate costs for a g-beam stacker can be found from the following scaling arguments. Mirror substrates are not likely to vary significantly in cost because their volumes are sinilar. The mirror area increases by about 128 to accomodate oversizing, and the coating costs increase proportionately, but polishing and grinding costs should not increase significantly because the increased area and increased mirror quality for the longer beam Iines will be offset by the ease of polishing smaller mirrors and the decreased influence of backup-bending distortion. To offset fluorine depletion, the 9-beam amplifier will have a 408 larger aperture, which will il,-rease the fused-silica costs by a corresponding amount. This is the most significant cost driver that favors the four-beam stacker. The larger array for the secondary mirror, and the additional hardware for mounting 9 instead of 4 mirrors per array, would increase the array costs about 108. The inaividual mounts for the delay micrors are less expensive, but 2-1/4 times as many are required, for a net cost increase of $30 \%$. Finally, the 9-beam stacker requires larger sensor foreoptics, which increase the cost by 58 per sensor. Even worse, the 9-beam stacker uses its sensors inefficiently, requiring 117 sensors instead of only 57, because 4 additional senso:-s are requiced on the preamp input and 2 more at each primary and tertiary array. However, the sensors at each primary and tertiary array can share a common set of signal-processing electronics, resulting in an overall cost increase of $66 z$ for sensors.

Overali, the cost for a 9-beam stacker is about $30 \%$ greater than for a 4-beam stacker wher small increases in other items are included for the 9-beam stacker. 
Table 2.4-1. Optical-system cost sumary (k\$).*

\section{I tem}

1. Aluminum mirror substrates.

2. Fused-silica windows and slumping.

3. Mirror and window polishing.

4. Dielectric coatings.

5. Amplifier and Raman-cell window mounts.

6. Delay $\pi$ i ror mounts and servo drivers.

7. Aligned mirror arrays, mounts, and servo drivers.

8. Laser diodes and drivers.

9. Aligment sensors, signal processing, and controls.

20. Alignment monitor and display and cable.

11. Power and zontrol cables installed.

12. Spaceframe installed.

13. Installmnt and rough alignment of optics. 14. Einal ali inment and test.
$2.45 \mathrm{x}$

Telescope

875

32,100

11, 500

3,500

900

1,100

7,000

400

9,300

200

400

8,000

1,400

300

Total

76,675

*System costs for 14 four-beam 2.45X-magnification stackers pumpiniJ a Lackward-scattering Raman cell. Research and development costs and front-end systems are not included. These are direct costs only: total does not include overhead and Ircofit, capital production, or test equipment but does include special jigs and tooling, in most cases. Most cost estimates are based on the volume requir, 1 and on the use of typical off-the-shelf components whenever prossible, but 7 few costs are for one-of-a-kind hand-produced items. The above costs are for an unoptimized system and could change if systrms assumptions are modified. 
2.5 Technology Development

The $10^{7}$ to $10^{8}$ shot lifetimes required for multilayer dielectric reflectors are orders of magnitude beyond the state-of-the-art at 248 no with $5.86 \mathrm{~J} / \mathrm{cm}^{2}$ and 100-ns pulse Iength. In addition, the segmented pivot and Raman-cell mirrors have joints that are subject to fluences up to $5.28 \mathrm{~J} / \mathrm{cm}^{2}$. The best comercial coatings have survived 100 shots at $248 \mathrm{~nm}$ with a fluence of $2 \mathrm{~J} / \mathrm{cm}^{2}$ and a pulse length of $20 \mathrm{~ns}$, while bulk-damage levels have been measured above $10 \mathrm{~J} / \mathrm{cm}^{2}$ for single shots at similar wavelengths. The present coatings are all limited by their linear-absorption coefficient and by the large number of defects $\left(\sim 10^{3} / \mathrm{mm}\right)$ with diameters of the order of a wavelength. For this report, it was assumed that the coatings could be produced by processing the stock to remove metal. ions and trapped gases (which sputter during deposition and increases the roughness) and by using dedicated ultrahigh-vacuum (uhv) ceposition chambers. If this procedure is inadequate, it may be necessary to use on]y materials that san be deposited in atomic layers by molecular-beam epitaxy and to develop this technique for large-area substrates. Another unlikely possibility is the development of two different-index glasses that can be compatibly deposited and annealed. Finally, if the Raman-cell mirror separation is groztly increased, it would be possible to angularly multiplex tne pump beams in the Raman cell, an action that would reduce the fluences in the $\mathrm{Kr} F$ optical line by a Eactor of two to four.

A second technology problem that carries great cost benefits is to reduce the thickness of the spherical-shell amplifier window to the minimum needed to withstand the amplifier overpressure. Calculations must be done to determine the coupling strength between the amplifier overpressire wave and the radially traveling shear wave that causes fracture. Cost and the convenience of fabrication dictated a four-pane window design for the amplifier, but the additional surfaces may result in reduced fracture resistance, which could drive the design back to a single-pane window. Mounts for these windows have been made for deep-ocean submersibles.

There will be some background photon noise from the afterglow of the windows and mirrors, there will be shot noise in the laser diodes, and there will be scintillation and angle-of-arrival of fluctuations from turbulence in the beain-path gas; consequently, either the detector may have to be modulated to increase the $S / N$ or it will be necessary to develop higher-power diodes with narrower divergence angles. The 
anglt-of-arrival fluctuations given by

$$
\left\langle a^{2}\right\rangle=2.91\left(\frac{3}{8}\right) L d^{-1 / 3}\left(1.340 \times 10^{-5}\right)\left(\rho_{\mathrm{O}} k_{G D}\right)^{2} C_{T}^{2}
$$

for spherical waves at STP should dominate the other disturbances. For the maximuu viewing distance of $L=290 \mathrm{~m}$ and a sensor of diameter $d=0.28 \mathrm{~m}$, we would like to keep $\left\langle a^{2}\right\rangle^{1 / 2}$ less than the resolution of the alignment system, $3.57 \mu \mathrm{rad}$, by specifying a gas of density $\rho_{0}$ and Gladstone-Dale constant $k_{G D}$ and by placing $a$ realistic bound on the atmospheric-temperature structure constant $C_{r}^{2}$. For argon, $\rho_{9}=1.7837 \times 10^{-3} \mathrm{~g} / \mathrm{cm}^{3}, \mathrm{k}_{\mathrm{GD}}$ $=0.1575 \mathrm{~cm}^{3} / \mathrm{g}$ at $589 \mathrm{~nm}$, and $\left\langle a^{2}\right\rangle^{1 / 2} \sim 3.6 \mu \mathrm{rad}$ if $\mathrm{C}_{\mathrm{T}}^{2}=0.025$ $\mathrm{K}^{2} \mathrm{~m}^{-2 / 3}$, or a temperature fluctuation of about $0.1 \mathrm{~K}$ across the sensor aperture dimensions, which might be hard to maintain near mirror edges or around the amplifier.

If helium with $p_{0}=0.1785 \times 10^{-3} \mathrm{~g} / \mathrm{cm}^{3}$ and $k_{\mathrm{GD}}=0.2017 \mathrm{~cm}^{3} / \mathrm{g}$ at $589 \mathrm{~nm}$ is used instead of argon, then $\left\langle a^{2}\right\rangle^{1 / 2} \sim 3.6 \mu \mathrm{rad}$ if $c_{\mathrm{T}}^{2}=$ $1.54 \mathrm{k}^{2} \mathrm{~m}^{-2 / 3}$, or about $0.8 \mathrm{~K}$ across the sensor aperture, which is quite reasonable. Therefore, helium should be used as the beam-path gar.

The servos and two-axis tilting mounts required for the individual delay mirrors need only be modest extensions of off-the-shelf components to handle the $87.5-\times 87.5-\mathrm{cm}$ mirrors, which have a total mass of $220 \mathrm{~kg}$. These mounts can be driven by a:railable motors that have a stepping rate of $2000 \mathrm{steps} / \mathrm{s}$ and stepping accuracies of $0.25 \mu \mathrm{rad} / \mathrm{step}$.

The four-mirror arrays present two more difficult problems: can the array be prealigned and held fixed, and can such a large mass be steered with the required accuracy? Calculations show that a highly damped honeycomb optical table can be used as a backup structure that will hold the mirrors to $\pm 5 \mu \mathrm{rad}$ quite easily, even in the face of vibrational impulse loads. The array will weigh about $3000 \mathrm{~kg}$ and must be designed so that it has no static friction in $i$ ts servo driver. It appears that a number of reactionless mirror mounts can handle this weight and that a servo can be designed to accurately drive the array over the required dynamic range; however, the response time may be rather slow. The servos for the alignment sensor itself are all of $\mathrm{f}-\mathrm{the}-\mathrm{shelf}$ components. The cycle time of the alignment system is about $40 \mathrm{~s}$, but longer times might be required if the $S / N$ of the sensor requires modulation or if the array servos require longer settling times. 
The mirrors and windows in the KrF and Raman optical trains are listed in Table 2.5-1, together with the energy fluence on each optical surface. The loss on retlection from a mirror was assumed to be 48 per bounce, the scattering was assumed to be 15 per bounce, the absorption loss in antireflection-coated fused-silica amplifier windows of 10-cm thickness was assumed to be 1.38 per pass, and the scattering loss was assumed to be 1.78 per pass. The fused-silica Raman-cell windows are tilted in a horizontal pl.ane at Brewster's angle, $56.38^{\circ}$, and the system is assumed to deliver a p-polarized beam to the Raman cell. The optical system delivers the beam from the KrF amplifying medium to the Raman cell with 828 efficiency, including a l.458 loss to Rayleigh scattering in the helium bean-path gas.

The mirror substrates have investment-cast aluminum backup structures, which include heat exchangers, water manifolds, and lightweight cast honeycomb to provide stifeness, dip brazed to a solid-aluminum faceplate that. is 1 to $2 \mathrm{~cm}$ in thickness. During the dip-brazing operation, water-manifold rear covers that contain water-pipe connections are also brazed to the investment casting. The overall mirror structure has approximately a $6: 1$ edge-to-thickness ratio (for square mirrors), which is sufficient to provide stiffness for polishing and to control mourting distortions. The thickness of the faceplate is determined by requiring the heat-exchanger pressure and thermal-mapping distortions to be negligible.

The mirrors must handle a thermal load of up to $0.47 \mathrm{~W} / \mathrm{cm}^{2}$. Gas cooling is inadequate to handle this load without producing excess backup-bending or overheating the mirror coatings. With a large-channel water-cooled mirror, the water only inseases in temperature by $\mathrm{I} \mathrm{K}$ in traversing a $100-c m$ cooling channel at a velocity of $1 \mathrm{~m} / \mathrm{s}$ and has a pressure drop of $2300 \mathrm{~N} / \mathrm{m}^{2}$. Under these conditions, the backup-bending curvature is only $10^{4} \mathrm{~m}$, which is comfortably large compased to the radius of curvature of the powered mirrors in the system. About l:0 litre/s of water are required to cool all the mirrors in the system; this water needs to be cooled about $1 \mathrm{k}$ before return, and the temperature control should be within $\pm 0.1 \mathrm{~K}$.

The fused-silica windows for the anplifier are $10 \mathrm{~cm}$ thick with a 6- to $8-\pi$ radius of curvature that is convex toward the amplifier to withstand pumping shocks. The 10-cm thickness is the upper limit set by the temperature rise the coatings can withstand Erom absorption of the RrF beam and the anplifier afterglow at shorter wavelengths. The temperature rise is also 
Table 2.5-1 Optical-train component number and fluence-loading sumary.

Number of

mirrors/windows

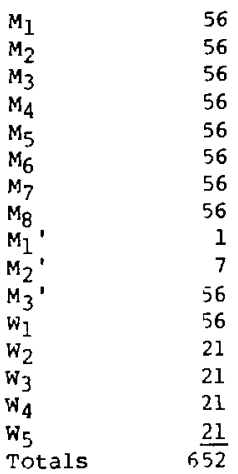

Number of sensors:

$s_{1}$
$s_{2}$
$s_{3}$
$s_{4}$
$s_{5}$
$s_{1}$

Total
Number of

arrays

14

14

14

IA

$-$

14

14

$-$

$-$

14

14

1

$\begin{array}{r}1 \\ 1 \\ \hline 116\end{array}$
Incident fluence $248 \mathrm{~nm} 268 \mathrm{~nm}$ $100 \mathrm{~ns} \quad 20 \mathrm{~ns}$ $\mathrm{J} / \mathrm{cm}^{2} \quad \mathrm{~J} / \mathrm{cm}^{2}$

2.04 *

i. 92

1.80

2.13 *

5.86

5.57

5.29 *

4.51

- 4.00

-

0.03

$-$

4. 04 *

2.72

2.44

2.32

0.55

* 400 ns

* Ignoring mirror tilts. 
limited because of the low thermal conductivity of fused-silica and the low heat-transfer coefficient for gas cooling of the window faces.

From an optical standpoint, there are three major uncertainties that might prevent the functioning of this system: dielectric damage, fracture of the amplifier windows, or unacceptable irradiance spread or beam jitter in the Raman beam line. The energy fluences must be quite high for this system to operate efficiently, and it may be difficult to get practical shot lifetimes at officient fluence levels; hence, the system is very sensitive to the development of coatings. The amplifier windows must be close to the amplifying medium or the gas-cleanup energy requiremenis will destroy the system efficiency, but, at these close distances, the window coatings court be damaged by the electron pumping beam or the window could be fractured by the overpressure wave. The difficulties with beam quality in the Raman cell are a combination of the typical OPDs for large metal mirrors, the eight glass surfaces it must traverse, the turbulence distortion resulting from energy deposited in the Raman medium itself, and any vibration of the optical elements. These difficulties do not appear to represent an insurmountable obstacle so long as extremely small, uniform focal spots are not required, but their solution might substantially increase the capital cost of the system.

There are also issues about the cycling time of the alignment system and the vibration of the window and mirror at the power amplifier. These issues will probably not affect the system directly in cost or complexity, but solutions to these issues could result in very substantial development costs.

polishing the mirrors under equilibrium pressure loads is a common procedure, but, to our knowledge, the inclusion of an equilibrium thermal load has not been tested. Cimputations and tests should be conducted to determine the optical-figure requirements for the unconventional stacker optics and to determine the least costly production method. Diamond turning under thermal load followed by ion polishing or computer-controlled minipad polishing to remove the machining grating might be developed to achieve the reguired figure accuracies at substantial savings of cost and time. It is possible that the mirrnr figures required for peliet illumination cannot be achieved on aluminum substrates, and the Raman mirrors $\mathrm{M}_{2}$ and $\mathrm{M}_{3}$ might have to be replaced with molybdenum-substrate nirrors. This replacement would increase the cost estimate for the substrates by almost a factor of three.

Finally, the present design assumes that the alignment drift is siow enough compared to the cycle time (40 s) of the alignment system that the beam 
prints renain within the mirror oversizing region, $\pm 100 \mu$ rad. Generally, this assumption will work if the optics are mounted directly on the floor and the building is constructed in a quiet environment, but the 2-kz cycling of the pulsed-power system directly affects both the amplifier window and the secondary mirror and could excite building resonances that could affect the rest of the optical train. The oscillation amplitude and spectrum of the containient building must be measured to determine what isolation of the optics is necessary or if a faster cycling of the alignment system would be sufficient.

\section{6 Conclusions}

From an optical standpoint, the key issue is the develoment of high-fluence dielectric coatings. This coating development is a long-lead-time item and must be resolved. Similarly, any issue such as surface quality or mounting distortion that is intrinsic to the large size of the optics is a long-lead-time item because large optics (particularly the fused-silica or molybdenum substrates for the mirrors) are time extensive. When these issues have been resolved, it will be practical to build a small engineering demonstrator to test other issues, such as beam-path conditioning, vibration sensitivity, window shock fracture, alignment and servo performance, and the s:-stem's soítware and signal processing. We believe the inclusion of a Raman cell fur veam cleanup and isolation in a fusion pulse-stacker system may ofEsct the disadvantage of developing high-fluence coatings, because the efficiency of peliet implosion may have a strong benefit/penalty trade-off for wave-front quality that would be beyond the capability of pure pulse stackers. 
Appendix 3. Process Systems, Facilities, and Capit=? Cost

J. Caird, W. O. Allen, and H. G. Hipkin

Bechtel National, Inc. 


\section{Contents}

\section{Section}

page

Fiqures

$3-i v$

Tables

$3-v$

3.1 Introduction and Summarv

3-1

3. 2 KrF Amplifie: Gas Flow Systems

$3-3$

3.2.1 General Considerations

3-3

$3.2 .2 \mathrm{Kr} F$ Amplifier Window

3-6

3.2.2.1 Co"siderations for a Solid window

7. 1

3.2.2.2 Considerations for a Purely Aerodynamic Window

3-11

3.2.2.3 Considerations for a Gas

$$
\text { Buffered } w^{*} \text { : ndow }
$$

$3-11$

3.2.3 Flurine separation Systems

$3-12$

3.2.3.1 Crvogenic Practionation

$3 \cdot 13$

3.2.3.2 Separation by absorption

$3-18$

3.2.3.3 Chemical Separation

$3-18$

3.2.3.4 conclusion on Fiuorine Sesparation

3-22

3.2.4 Other Flow Design Considerations

$3-22$

3.2.4.1 Construction Materizis

$5-23$

3.2.4.2 Heat Removal

3-23

3.2.4.3 Pressure-Wave Damping and Flow Management

$3-24$

3.2.4.4 Pressure Drops and Blower Power

3- 25

$3.2,4.5$ Cooling water Flow Power

$3-26$

3.2.4.6 Laser Gas Cieanup

$3-26$

3.2.4.7 startup and shutdown operations

$3-27$

3.2.4.8 Flow System Layout

$3-28$

$3-28$

3. 3 Methane Ramar Cell Gas Flow systems

3.4 Overall svstem Fnergy output and Efficiency

3.5.1 Stacker Beam Channel Design

3.5.2 Ferlmeter Amplifier system Housing

3.5.3 Hub Builaing Desiqn

3.5.4 Raman Compressor Building Design 


\section{Contents (continued)}

Section

Page

3.6 Facility Construction Cost Estimate

3.6.1 General

3-41

3.6.2 Pricing Levels

$3-42$

3.5.3 Direct Field Construction Costs

$3-42$

3.6.3.1 Mechanical Equipment

$3-42$

3.6.3.2 Materials

$3-43$

3.6.3.3 Construction Labor

$3-43$

3.5.4 Indirect Ficid Costs

$3-43$

3.6.5 Front End Costs

$3-43$

3.6.6 Home Office Servires

$3-43$

3.6.7 Contingency

$3-45$

3.6.8 Desiqn Assumptions

3-45

3.6.9 Exclusions

3-45

3.5.10 Estimate Summary (400-ns Pulsed Power)

$3-46$

3.6.11 900-ns System Costs

$3-46$

$3-48$

3.7 S. mar. at Technology Development Issues

$3-51$

3.7,1 ETF Desiqn Philoscphy

$3-51$

3.7.2 KrE Final Amplifir.r Design

$3-53$

3.7.3 Optical System Derign

$3-54$

3.7.4 Pulsed-Power System Design

$3-56$

3.7.5 Other Facility Issues

$3-57$

3.8 Conclusions

$3-59$

3.9 R.eferences

Page

Figure

Eigures

3.2-1 KrF amplifier cavicy illustrating the postwhot 3-7 expansion of the active region due to e-beam heating.

3.2-2 Process-flow diagram for $F_{2}$ separati on from the $3-15$ window buffer gas (Ar/Kr mixture or pure $\mathrm{Kr}$ ) by crvogenic fractionation.

3.2-3 Process-tlow diagram for $F_{2}$ separation from helium buffer gas hy flash separation. 
Figures (continued)

Fiqure

Page

3.2-4 Process-flow diagram for $F_{2}$ separation from the 3-19 window buffer gas by selective absorption in a suitable solvent.

3.2-5 Process-flow diagram for chemical separation of $\mathrm{F}_{2}$ from the window-buffer gas.

3.2- K KrF amplifier gas-Elow system. 3-29

3.5-1 Individual stacker beam channel. $3-34$

3.5-2 Perimeter building elevation. 3- 37

3.5-3 Fusion laser building plan. 3-39

$3.7-1$ Alternate stacker design using pre-delayed front$3-55$ cnd input and individual Raman amplifiers for each stacker module.

\section{Tables}

Table Page

3.2-1 YrF amplitier design parameters (400-ns pulsed $3-4$ power).

3.3-1 Methane Raman compressor design parameters. 3-30

3.4-1 Laser system output and efficiency. 3-35

3.6-1 Buildings and ancillary equipment costs (MS) 3-44 (400-ns pulsed power).

3.5-2 KrF fusion laier system costs (M\$) (400-ns pulsed power).

3.5-3 Buildings and ancillary equipment costs (MS) (900-ns pulsed power).

3.5-4 KrF fusion laser system costs (M\$) $3-50$ (900-ns pulsed power). 
Appendix 3. Process Systems, Facilities, and Capital Cost

\subsection{Introduction and Summary}

To complete the laser system, equipment must be added to proper?.y condition the gas media in the KrF laser amplifiers, the Raman amplifier, and the beam-propagation paths. Electrical power and cooling systems, buildings to house the entire system must be designed. The purpose of this chapter is to define these additional system requicements and to present an overall system capital cost estimate.

One major task in this phase of the study was to examine the feasibjlity of using aerndynamic windows for the $\mathrm{KrF}$ laser amplifiers and the methane Raman amplifier. It was thought that aerodynamic windows would eliminate the requirement for solid windows with high-damage-threshold antireflection coatings. Since the damage threshold of currently available optical coatings at the $\mathrm{KrF}$ laser wavelength is considerably lower than that required by this sustem, this would eliminate the need for an intensive coating development proqram.

Several approaches to using aerodynamic windows for the rif amplifiers were examinet in depth and are presented in section 3.2. However, none of thesc approaches was found sufficiently attractive to incorporate in the final design. The primary reason for this result is that fluorine from the active laser gas mixes with, and must be removed from, the gas in the aero-window. The guantity of fluorine involved is such that it: removal is energetically prohibitive (from a laser efficiency point of view) for all methods considered. It was also concluded that a solid window is probably required in any case in order to seal off the active laser region containing highly reactive fluorine and highly expensive krypton gases from the rest of the iaser system. Additional potential options for an aero-window system design are sugyested for further study, in the event that such a system is ultimately required.

A design for the $\mathrm{KrF}$ amplifier gas flow system without an aerodynamic window is presented in section 3.1. Gas-flow conditioning equipment--including the gas cleanup system, heat exchanger, ind blower--is discussed. Systems and procedures for startup and shutdown are included. The major pressure drop around the flow loop, which determines the reguired blower power, occurs in the heat exchanger. It is assumed that additionai flow manaqement for suppression of gas turbulence and acoustic pressure waves can 
be accomplished without significant additional pressure drop. This assumption is justified because the Raman amplifier is a very large target: the divergence of the $\mathrm{RrF}$ beams can be very large without significant loss of efficiency. An adequate quantitative analysis of the bean divergence createa by medium inhomogeneity far above the diffraction limit is needed to establish system requirements in this area.

Flow systems for the methane Raman cell are described in Section 3.3. For this system, it was concluded that antireflection coatings are not required if the laser beams are polarized and the winoows oriented at Brewster's angle. The surface damage threshold of clean, highly polished, uncoated fused silica is believed to be adequate for this application. Also, analysis has shown that self-focusing beam distortion due to the nonlinear refractive index of quartz is negligible, given the long pulsewidth assumed in this study a.d a reasonably thin window. Brewster-angle windows were not considered for the KrF amplifiers in this study because of geometrical constraints of the initial design. However, it appears that there are ways in which they could be incorporated in future designs, and further consideration is suggested.

A conceptual design for the building architecture is presented in section 3.5. The need for controlled atmospheric conditions in the beam-propagation paths is discussed, and a design approach is described. Additional equipment for cooling the e-beam generator components is discussed, along with other Jeneral facility requirements (site work, etc.).

An order-of-magnitude cost estimate for systems based on 400-ns and 900-ns pulsed power is given in section 3.6. The estimate includes all major mechanical and electrical equipment, building materials, conscruction labor, indirect field costs, home office services, and 258 contingency.

Several technical issues were identified during the study for which adequate design solutions have not yet been proven. A number of these are discussed in section 3.7. It is hoped that resolution of these issues will not have a significant impact on overall system costs. Some suggestions for design improvements are also included in this section.

The general design of systems described in this chapter would be applicable for use with KrF amplifiers that have e-heam pump pulse durations from a few hundred nanoseconds to a microsecond or more. The design of a system using 400-ns $\mathrm{PFL}$ e-beam generators is discussed in detail to establish a point design. The modifications appropriate for use of 900-ns PFN pulsed power are discussed briefly in section 3.6.11. 


\section{2 KrF Amplifier Gas Flow Systems}

\subsubsection{General Considerations}

The basic design parameters for the KrF laser amplifiers were established by LLNL at the outset of this study. Table 3.2-1 lists a number of those parameters that are relevant to the design of the anplifier gas-flow systems. These choices are consistent with the basic design goals, which include an output energy fluence of $4 \mathrm{~J} / \mathrm{cm}^{2}$, a pulse width of $400 \mathrm{~ns}$, and the largest possible amplifier size consistent with parasitic mode control.

Additional specifications needed to design the jas flow system include the following:

- Ninety percent of the deposited e-beam energy, or $1.44 \mathrm{MJ}$ per shot, must be removed as waste heat. The actual waste heat will be somewhat less, because some of the energy will be radiated to the walls and out of the wi.rdow (in the form of nonstimulated $\mathrm{KrF}^{*}$ fluorescence, $\mathrm{ArF}$ fluorescence, and fluorescence of wher excimers and excited atomic species). Therefore, the assumption that $1.44 \mathrm{~m} / \mathrm{shot}$ turns into heat will lead to a conservative design for the required heat exchanger, and for any structural components that must handle the pressure wave resulting from the heat adition.

o The ambient temperature of the gas prior to e-beam deposition is $50^{\circ} \mathrm{C}$ to facilitate subsequent heat removal.

- An active region clearing factor (or flush factor) of 2 is required to raestablish sufficiently quiescent conditions in the amplifier cavity. Given a pulse repetition rate of $2 \mathrm{~Hz}$, this leads to a required flow velocity of $8 \mathrm{~m} / \mathrm{s}$ through the cavity.

The additional design parameters are also included in Table 3.2-1.

The KrF amplifier flow system design must contend with the temperature 1 ise and pressure wave resulting from the waste heat left in the active region after each shot. The initial temperature rise occurs before the gas has time to expand significantly in volume, thus the increase is given by

$$
\Delta \mathbf{T}=\frac{\Delta \mathrm{U}}{\mathrm{C}_{\mathrm{V}}}:
$$

where $\Delta U$ is the increase $j$ in internal energy, and $C_{v}$ is the specific heat of the gas at constant volume. Since the laser gas is essentially an ideal monatomic gas, the specific heat is

$$
c_{v}=\frac{3}{2} n R \quad
$$


Table 3.2-1

KrF amplifier design parameters

(400-ns pulsed power).

\author{
Aperture \\ Active length \\ $\mathrm{Ar} / \mathrm{Kr} / \mathrm{F}_{2}$ mixture \\ Pressure \\ Electron energy \\ E-bearn energy \\ Intrinsic efficiency \\ Cutput energy (optical) \\ Stacker compression factor \\ Dutput pulse wideh \\ Repetition rate \\ Waste heat \\ Gas inlet temperature \\ Clearing factor \\ Gas flow rate
}

$2 \times 2 m$

$3 \mathrm{~m}$

$0.937 / 0.060 / 0.003$

1 atm

1. $3 \mathrm{MeV}$

$1.6 \mathrm{MJ} / \mathrm{shot}$

108

$16 \mathrm{JJ}$

$4 x$

$100 \mathrm{~ns}$

$2 \mathrm{~Hz}$

1. $44 \mathrm{MJ} / \mathrm{shot}$

$50^{\circ} \mathrm{C}$

2

$8 \mathrm{~m} / \mathrm{s}$ 
where $\mathrm{n}$ is the number of moles of gas in the laser cavity, and $\mathrm{R}$ is the universal gas constant. Using Eq. (3-2) and the ideal gas equat: on of state, Eq. $(3-1)$ can be rewritten

$$
\frac{1 \mathrm{~T}}{\mathrm{~T}_{\mathrm{O}}}=\frac{2}{3} \frac{\mathrm{U}}{\mathrm{P}_{\mathrm{O}} \mathrm{V}_{\mathrm{O}}}
$$

where $\mathrm{P}_{0}, \mathrm{~V}_{\mathrm{O}}$, and $\mathrm{T}_{\mathrm{O}}$ are the pressure, volume, and temnerature of the active region prior to the energy deposition. At 1 atm $P_{0} V_{O}=\left(1.013 \times 10^{5} \mathrm{nt} / \mathrm{m}^{2}\right) \cdot(2 \times 2 \times 3 \mathrm{~m})=1.22 \times 10^{6} \mathrm{~J}$. With $\mathrm{iv}$ $=1.44 \times 10^{6} \mathrm{~J}, \mathrm{Jg} \cdot(3-3)$ yields $\Delta \mathrm{T} / \mathrm{T}_{\mathrm{O}}=0.79$, corresponding to a temperature rise of $255^{\circ} \mathrm{C}$, and a corresponding pressure rise of 0.79 atm. As noted above, the actual temperature and pressure $r$ ise will be smaller because some of the excess e-bean energy will be radiated away.

Since the pressure outside of the active laser region remains at 1 atm, the increased pressure inside will cause the active region to expand. If the cavity is constructed so that the active region can expand freely, the increase in its volume can be easily estimated. The expansion will continue until the internal pressure tecomes equal to the 1-atm pressure of the rest of the plenum (the gas plenum volume is assumed to be very large zompared to that of the amplifier cavity, so that the overall pressure $r$ ise is negligible). Assuming negligible heat transfer between the expanding gas and the surrounding medium, the internal energy is reduced only by work done against the constant external pressure, hence

$$
d U=-P_{0} d V=c_{v} d T
$$

Integrating Eq. (3-4) and using the ideal gas equation of state yeilds

$$
\frac{V_{f}}{V}=1+\frac{2}{5} \frac{\Delta U}{P_{0} V_{O}},
$$

where $v_{f}$ is the final expanded rolume of the active region. In the present system $v_{f} / v_{O}=1.47$, ndicating a 478 increase in the volume of the active laser gas.

It is of interest to determine the increase in the linear dimensions of the active region. Eor this purpose, it is assumed that the gas expands equal 
distances in the dinensions normal to the e-beam (the e-beam foil prevents expansion in its direction). This situation is depicted in Fig. 3.2-1. The final volume is given by

$$
v_{f}=x_{E} y_{E} z_{E}=\left(x_{o}+\Lambda\right)\left(y_{o}+\Delta\right) z_{o}=\alpha v_{o}
$$

Solving Eq. (3-6), given the parameters of the current system yields $\Delta=$ $0.515 \mathrm{~m}$. This amounts to a 268 expansion in the flow direction and a $17 \%$ expansion in the direction of laser beam propagation, as shown in the Fig. 3.2-1. The actual expansion of the active region will be a complex and turbulent process, but these figures give a reasonable estimate on which to base the system design.

The temperature of the active region drops as it expands and does work on the gas in the plenum. The reduction in temperature is given by

$$
\Delta T^{\prime}=\frac{P_{0} \Delta V}{C_{V}}=\frac{2}{3}(a-1) T_{0}
$$

where $\alpha$ was the volunetric expansion factor. For this design $\Delta \mathrm{T}^{\prime}=102^{\circ} \mathrm{C}$, yielding a final typerature in the active region of $203^{\circ} \mathrm{C}$. The internal energy of the active region is reduced by $\Delta \mathrm{U}^{\prime}=\mathrm{C}_{\mathrm{v}} \Delta \mathrm{T}^{\prime}=580 \mathrm{~kJ}$, leaving $860 \mathrm{~kJ}$ of excess thermal energy in the active region. The $580 \mathrm{~kJ}$ released during the expansion process is assumed to be radiated in the form of sound waves that are dissipated in acoustic absorbers and in the walls of the plenum. This energy must still be removed as heat, but its distribution will depend on the location of the acoustic absorbers.

\subsubsection{KrF Amplifier Window}

The angularly multiplexed beams require long propagation paths to allow appropriate time delays and amplifier fill factors. The propagation paths cannot contain fluorine because it absorbs Iight at the Iaser wavelength, and cannot contain much krypton gas because it is very expensive. The KrF amplifiers must therefore be isolated from the propagation paths by some form of window that transmits the beam and contains the gases. 


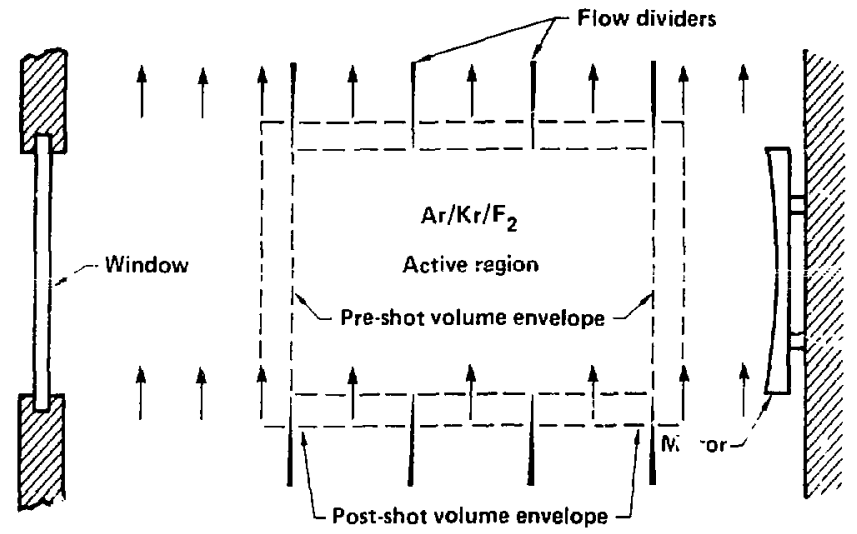

Fig. 3.2-1 KEF amplifier cavity illustrating the postshot expansion of the active region due to -beam heating. 
Three major options to the window design were considered:

- A solid transparent window close to the active region.

- A purely aerodynamic window.

- A solid window at a large distance from the active region with an aerodynamic buffer gas separating it from the active region.

\subsubsection{Considerations for a solid Window}

The solid window close to the active region has the advantage that it eliminates the necessity for coflowing gas streams, the conditioning equipment required for the coflowing window gas, and the energy expenditure required for such conditioning. However, the window (and amplifier mirror) must be separated from the active region by a finite distance so that it is not damaged by the e-beam. In this space, laser energy is absorbed by inactive Fluorine molecules in the ground state. The absorption cro s section ${ }^{1}$ $\sigma_{\mathrm{abs}}$ for $\mathrm{F}_{2}$ at the $\mathrm{KrF}$ laser wavelength $(248 \mathrm{~mm})$ is $1.5 . \times 10^{-20} \mathrm{~cm}^{2}$, and the 0.38 of $\mathrm{F}_{2}$ at 1 atm and $50^{\circ} \mathrm{C}$ yields a density of $W=6.9 \mathrm{z}^{1} \mathrm{j}^{16} \mathrm{~mm}^{-3}$. The resulting attenuation coefficient is $a=1.04 \mathrm{x}$ $10^{-3} \mathrm{~cm}^{-1}$, or about $10 \% / \mathrm{m}$. n addition, reflection losses from thi window surfaces must be accepted (abo: : 48 per surface if oriented normal to the beam), or the surfaces must be intireflection coated. Presently arailable antireflection coatings cannot withstans the ontical energy density assumed in this design without being damaged. A coating development program is required if normal incidence reflection losses must be eliminated (this may not be necessary for the ETF, or if Brewster windows can be used).

If the window is very close to the active region, there will be littie atienuation of the pressure wave generated by the e-beam. Since the window is a stationary surface, the wave will reflect off it, resulting in a local pressure at the surface of nearly twice the initial overpressure. The window m'dst therfore be des': $-\rightarrow$ to withstand the stress created by an overpressura of approximately 1.6 atm on its inner sir tiace.

Fused silica (quartz) appears to bu the most reasonable window material. If it is used a safe working tensile stress $\sigma=2000$ psi can be assumed. for a flat square window under static lcad conditions, the window thickness required to keep the stress down to this level is given by

$$
t=0.048 \mathrm{~L}[\mathrm{P}(\mathrm{atm})]^{\frac{\mathrm{L}}{2}},
$$


where $L$ is the length of a side, and $P$ is the pressure load in atmospheres. In this case, a thickness of $12 \mathrm{~cm}$ is calculated for the 2- $\times 2-m$ aperture. If the window is curved, a higher static pressure can be tolerated for a given thickness. However, the effects of dynamic loads and repetitive operation have not been analyzed in sufficlent detall. These effects are expected to increase the required window thickness.

The intrinsic absotption ccefficient for pure fused silica (as might be expected for suprasil) at $248 \mathrm{~nm}$ is $1.22 \times 10^{-3} \mathrm{cn}^{-1}$ (see Ref. 2). At a thickness of $10 \mathrm{~cm}$, the quartz will absorb a little over 18 of the beam in the bulk material, yielding a heat load of $0.1 \mathrm{w} / \mathrm{cm}^{2}$ for the average beam intensity of $B \mathrm{~W} / \mathrm{cm}^{2}$. Additional heat would be generated in the guart?. window through absorption of ArF" fluorescence $(193 \mathrm{~nm})$ from the actire region. The average intensity of $A r F^{*}$ fluorescence at the window is estimated to be about $0.2 \mathrm{~W} / \mathrm{cm}^{2}$. The attenuation coefficient at this wavelength is $0.016 \mathrm{~cm}^{-1}$, yielding an addr sat load of $0.03 \mathrm{w} / \mathrm{cm}^{2}$. Aditional fluorescence by other species increase the bulk tisermal

ing, sopucialy at shocler wavelengths, where guartz absogtion is irronger. $t$ is assumed that the total bulk heating load will be less than $0.2 \mathrm{~W} / \mathrm{cm}^{2}$ for a 10 -cm-thick window, corresponding to a volumeicric heat deposition rate of $\mathrm{n}=0.02 \mathrm{w} / \mathrm{cm}^{3}$.

The thermal conductivity of quartz is rather $10 \mathrm{w}, \mathrm{R}=1.4 \times 10^{-2} \mathrm{~W} / \mathrm{cm}$ $K$, and the bulk heat deposition will cause the center of the window to be hotter than the surfaces. The hotter interior expands, putting the sufaces under tension and the center under compressive stress. Assuming equal heat Elow out of both surfaces it can be shown that the temperature difference between the center and the surfaces is given by

$$
I T_{c}=\frac{Q t^{2}}{B K},
$$

where $t$ is the thickness of the window. In this case, a temperature $r$ ise of $1 \mathrm{~B}^{\circ} \mathrm{C}$ is calculated. Because the thermal expansion coefficient of ciartz is small, this temperature $r$ ise is not expected to be a problem; however, because $\Lambda T_{c}$ varies with the square of the thickness, a limiting value wijl eventually be reached if the thickness were substantially increased. Further analysis of the window's thermal stress behavior shuild be undertaken. 
It is assumed that the window is primarily surface-cooled by the gases flowing by it. For a gas velocity of $8 \mathrm{~m} / \mathrm{s}$, making conservative assumptions regarding the equivalent duct diameter, a heat transfer coefficient of $h=$ $1.33 \times 10^{-3} \mathrm{w} / \mathrm{cm}^{2}{ }^{\circ} \mathrm{C}$ is obtained for Argon gas. The $0.02 \mathrm{w} / \mathrm{cm}^{3}$ of bulk heating leads to a surface heat flux of $0.1 \mathrm{~W} / \mathrm{cm}^{2}$ per surface for a 10-cm-thick window. An antireflection coating that absorbs $10^{-3}$ of the beam Energy at eaci. surface adds another $0.01 \mathrm{w} / \mathrm{cm}^{2}$, yielding a total flux of $\phi=$ $0.11 \mathrm{~W} / \mathrm{cm}^{2}$ per surface. The temperature drop across the solid-gas interface is calcul ated from

$$
\Delta \mathrm{T}_{\mathbf{s}}=\frac{\varphi}{\mathrm{h}}
$$

which yields $83^{\circ}$ in this case (assuming equal heat cransfer on both sides of the window). Since antireflection coatings are rieposited at temr.eratures in excess of $300^{\circ} \mathrm{C}$, this temperatur 2 rise should be acceptable. However, if

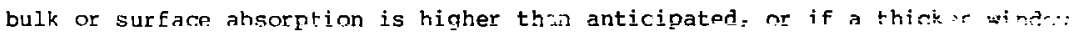
is required, additional cooling may be nacessary. Higher cooling rates could easily be obtained by flowing gas at a higher rate outside the window. If helium gas is used outside the window, the heat transfer coefficient would be increased dramatically. In any case it appears that the teriperature rise in the window is not a limiting factor even for a rather thıck window, provided the absorption coeficient is held to near the intrinsic limit for fused silica.

The structural integrity of a $10-c m-t r i c k$ fused silica window is doubtful for a 2- $x$ 2-m aperture, given the repetitive pressure waves incident on it. Doubling the thickness would improve chances of survivabilicy, and might be acceptable from the standpoint of thermal load, but would assenuially double the cost of this most expensive optical element. In addition, fabrication problems may be encountered for very thick large-aperiure windowe. It is felt that a more reasonable design approach would be to segment the window, rather than increase its thickness any further. The final design, therefore, assumes a four"piece window, each 1 x $1 \mathrm{~m}$ square, and $10 \mathrm{~cm}$ thick, supported by structural steel struts that are about $5 \mathrm{~cm}$ thick. 


\subsubsection{Considerations for a Purely Aerodynamic window}

If a purely aerodynamis window with no absorbing gas species is used, the optical attenuation and coating damage problems of the window are eliminated. However, as shown above, the deposition of energy in the active region causes significant mixing of the gas streams, so that fluorine must be renoved from the window gas. The energy required for such a separation operation must be compared to the energy that would be lost if a solid window were used. It must also be realized that no separation process could be completely effective, so that some $A r, K r$, and $F_{2}$ gas would remain in the wirdow gas stream and would eventualiy leak out into the propagation paths. This would require further systems for $F_{2}$ cleanup and $\mathrm{Kr}$ recovery, and might be unacceptable from a safety point of view.

The problem of separating fluorine from the window gas is crucial to the "iability of the aerodynamic window. In the following section, a system enrloyiny a solid window set at a substantial standoft distance from the active region is considered. The problem of keeping fluorine gas out of the

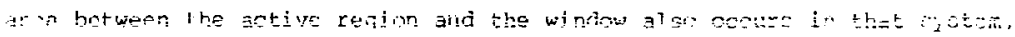
Further discussion of the fluorine separation systems required in either case appears in section $3.2,3$.

\subsubsection{Considerations for a Gas Buffered Window}

If a solid window is used close enough to the active region to limit fluorine absorption losses, it will have to be rather thick to withstand the pressure wave. Another potential design would reduce the reguired thickness by placing the window at a large distance from the active region, where the pressure wave is substantially attenuated. However, for reasonable standoff distances, this approach requires use of a gas buffer containing a substantially reducea concentration of fluorine between the active region and the window. Ir, this respect, this approach is similar to that of the pure aerodynamic window. However, the use of a solid window has the advantage that the beam propagation path is physically sealed off from the KrF amplifier region, allowing their atmospheres to be maintained completely independently. Discussion of the fluorine separation systens required to condition the buffer gas is presented in the next section. 


\subsubsection{Fluorine Separation Systems}

In the cases where a pure aerodynamic window or a gas buffered window are considered, the concentration of fluorine in the window gas or buffer gas must be kepc very low in order to minimize optical absorption losses. It has been shown that the absorption of fluorine at the $\mathrm{KrF}$ laser wavelength is about $108 / \mathrm{m}$ for the $\mathrm{F}_{2}$ concentration that exists in the active region 10.38 or $3,000 \mathrm{ppin}$ ). To reduce this to less than $18 / \mathrm{m}$, the fluorine concentration must be kept below 300 ppm.

In order to minimize mixing of the gas in the active region with the window or buffer gas, the flow velocity of the two regions must be matched at the boundary between them. However, as is shown above, the release of energy in the amplifier heats and expands the active gas so that substantial mixing of the gas streams is inevitable. The expansical takes place at approximately the velocity of sourd in the medium $(\sim 220 \mathrm{~m} / \mathrm{s})$, which is much faster than the transverse flow velocity $(\sim \mathrm{g} / \mathrm{s})$. It would appear reasonable to assure that all the fluorine that overflows the bounds of the active region must be subsequently removed from the buffer gas. From the previous analysis, this amounts to 178 of the $F_{2}$ initially in the active region for the present sistem design (see Fig. 3.2-1). This amounts to $8.9 \mathrm{~g} / \mathrm{shot}$, or $17.6 \mathrm{~g} / \mathrm{s}$ per amplifier. For the total system of 14 amplifiers, this amounts to $245 \mathrm{~g} / \mathrm{s}$ or $885 \mathrm{~kg} / \mathrm{h}(a \operatorname{lmost} 1$ ton $/ \mathrm{h}$ ). It should be noted that this assumes a buffar gas on both sides of the active ragion. It may be possible to bring the mirror in close to the active region so that a buffer gas is not necessary on that side; in this case, the amourt of fluorine to be recycled would be cut approximately in half.

For the purpose of performing some de-ign calculations, it was assumed that only the buffer gas in the $50-\mathrm{cm}$ region immediately adjacent to the active region (on both sides) needs to be cleaned, while the rest of the buffer gas is simply recycled. Because this region contains mostly buffer gas from between shots and from outside the expanded active region, the avezage concentration of $F_{2}$ in this stream is on the order of 1000 ppm (0.18). The problem of recovering fluorine at the rate of 1 ton/h from a stream where its concentration is fairly low to begin with is formidable, as discussed below.

The options for removing fluorine from the buffer gas include cryogenic fractionation, absorption, aojsorption, permeation, chemical removal, and thermal diffusion. Of these, thermal diffusion, permeation, and adsorption can readily be eliminated from further consideration. Thermal diffusion and 
permeation require develcpment programs before they can be utilized. Adsorption is an accepted incustrial technique, but fluorine is so reactive that it will react with any common adsorbent. Development of an inert adsorbent would require a lengthy development program. The remaining three techniques are considered in more aetail in the following paragraphs.

\subsubsection{Cryogenic Fractionation}

Fractionation at cryogenic temperatures is a well-developed industrial technology used mainly for air separation. The energy reguired for the separation 's input through compression of the feed gas and through refrigeration from an external system (if autorefrigeration is not used). The separation process depends strongly on the assumed composition of the butfer gas. Three cases were considered in this study: an Ar/kr nixture in ratio 94,6 , a pure Kr buffer, and a pure He buffer.

The AL/Kr buffer mixture has essentially the same specific glavity and viscosity as the active gas and therefore minimizes preshot turbulent shearing brtwren: the actlve and huffer gas regions. However, fluorine and argon hoil at temintatures that are onlv $2.3 \mathrm{~K}$ apart, so the separation of fluorine is very difficult. In addition, the freezing points of argon and krypton are within $4 \mathrm{~K}$ of their boiling points at atmospheric press $\mathrm{re}$. In order to keep krypton from solidifying in the equipment, all temperatures need to be kept abo'e $116 \mathrm{~K}$. pure fiuorine does not liquefy at atmospheric pressure until the temperature is reduced to $85 \mathrm{~K}$, and--in order to make this fractionator work proper $l y-$-the column pressure must be increased lintil it liquefies abnve 116 K. This requirement dictates an operating column pressure of about 200 psia.

A lower limit to the power used by the compressor can be obtained by assuming isothetmal compression. To the extent that heat is not removed during the compression process, the increase in temperature of the gas results in higher pressure and therfore higher compressive power. From the ideal gas liw at constant temperature the work clone on the gas is given by

$$
w=-\int_{i}^{l} \mathrm{PdV}=n R^{\top} \int_{i}^{\mathrm{C}} \frac{\mathrm{dP}}{\mathrm{P}}=\mathrm{e}_{i} \mathrm{~V}_{i} \ln \left|\frac{\mathrm{P}_{\mathrm{E}}}{\mathrm{P}}\right| .
$$

The compressive power is given hy

$$
\frac{l W}{i t}=P_{i}\left(\frac{1 V_{i}}{I t}\right) \ln \left|\frac{P_{E}}{P}\right|
$$


where $\Delta v_{i} / \Delta t$ is the voluse of feed gas to be processed per unit tive. In this case the feed gas streal for each applifier consists of two ducts $500 x$ $2 \mathrm{~m}$ in cross section, with gas flowing at $B=/ 8$ giving $\Delta V_{i} / \Delta t=16 \mathrm{~m}^{3} / \mathrm{s}$. With $P_{i}=14.7 \mathrm{psia}\left(1.013 \times 10^{5} \mathrm{nt} / \mathrm{m}^{2}\right)$, and $P_{f}=200 \mathrm{psia}, \mathrm{Eq} .(3-12)$ yields $\Delta w / \Delta t=4.2 \times 10^{6} \mathrm{~W}$ per amplifier.

Allowing for scme inefficiency and lack of ideal lsothernal conditions, the actual compressor load will be somewhat higher. This figure would be cut i:l half if only one sitipstrean (the one on the window side) has to be processed. This amount of process energy consumption in itself looks prohibitive from the point of view of laser efficiency because it is comparable to the total 6 w per amplifier electrical input to the e-beans.

The Clow diagran for a heat exchanger-franctionator combination is shown in Fig. 3.2-2. Both of these major units, with their piping, would be built in a "cold box," packed with insulation, and purged with a dry gas. The feed gas (F) is compressed to colunn pressure before entering the exchanger in order to maintain the proper temperature differences. An external refrignration system is assuned. The fractionator was calculated to take overhead (D) an amount equal to the amount that expanded from the active gas into the buffer gas, and containing essentially all the fluorine. The overhead is returned to the active gas loop, while the flucrine-depleted bottoms (B) are returned to the buffer gas. Calculations were performed for a number of designs corresponding to various numers of trays and reflux ratios. The two best designs gave the following results:

Case I

Number of Trays

Feed Tray (from top)

Reflux Rate (lb moles/h)

$F_{2} i_{\text {il bottoms (ppm) }}$

$\mathrm{Kr}$ in Overhead

Refrigeration Load (w)

Refrigeration Temperature (K)
52

11

20,000

85

0

$30.5\left(10^{6}\right)$

116

\section{Case II}

52

11

10,000

290

0

$15.8\left(10^{6}\right)$

116

Neither of these designs appears practical because of the very high refrigeration loads. Case II represents a power input of about $32 \mathrm{ks}$ per amplifier, assuming a rel-igeration coefficient of performance of 0.5 : The problem with this design is the difficulty of separating the fluorine from the 


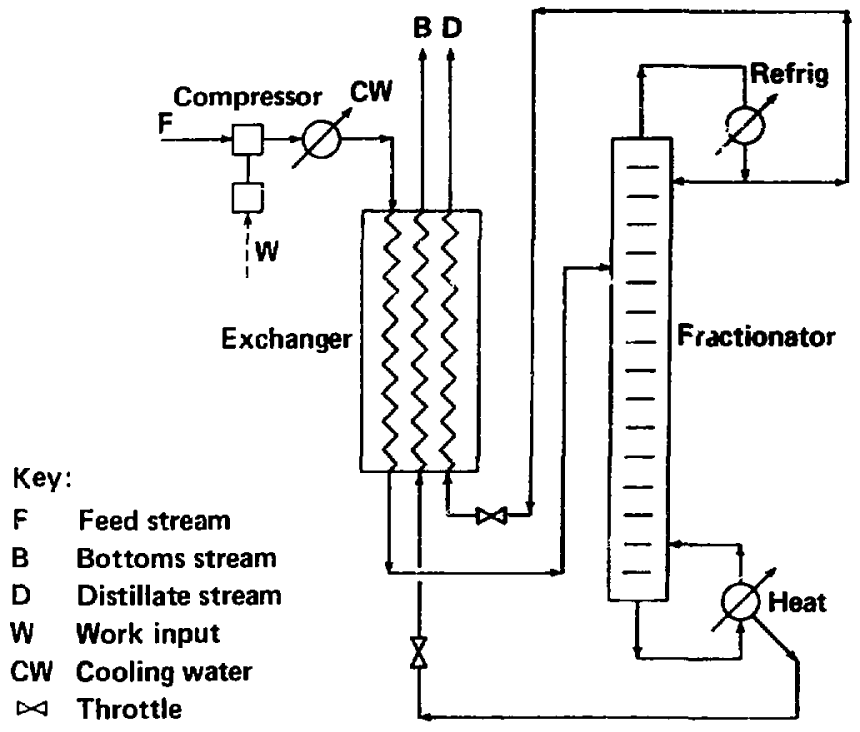

Fig. 3.2-2 Process-flow diagram for $F_{2}$ separation from the window buffer gas (Ar/Kr mixture or pure $K r$ ) by cryogenic fractionation. 
majority component, argon. However, the 290-ppa concentration of $F_{2}$ in the bottoms (Case II) Yields an absorptivity in the buffer gas of $0.13 / \mathrm{m}$, which should be acceptable for the buffered-window scenario.

Using pure krypton as a buffer gas simplifies the separations. Rigure 3.2-2 also serves as a flow diagram for this design. Here the overhead is designed to contain all the fluorine and no nore than 68 krypton. The overhead is recycled to the active gas, and the krypton bottoms are recycled to the buffer gas. Calculations were performed for five cases, with the best--but not necessarily optimu-giving the following reaults:

$\begin{array}{lc}\text { Number of Trays } & 22 \\ \text { Feed Tray (from top) } & 12 \\ \text { Reflux Rate (lb noles/h) } & 5008 \\ F_{2} \text { in bottoms (ppo) } & 0.22 \\ \text { Kr in Overhead (mole } z) & 6.4 \\ \text { Refrigeration Load (w) } & 5.9\left(10^{6} \text { ) }\right. \\ \text { Refrigeration Temperature (R) } & 131\end{array}$

While the use of a krypton buffer reduces the energy required for the separation significantly, the technique is still unattractive. The colum pressure of 200 psia still requires more than $4 \mathrm{kH}$ of compressor power, and the power input to the refrigerator is $12 \mathrm{~m}$ per amplifier, assuning a coefficient of performance of $\mathbf{0 . 5}$. This asount of input power would reduce laser efficiency by a fartor of alnost 4. In addition, the increased $\mathbf{k r}$ inventory used here would be quite expensive. As a result, the pure krypton buffer gas does not appear to be a practical alternative elther. It is possible that a fractionation system with a solid $k r$ or $\mathrm{Ar} / \mathrm{Kr}$ interfa ie with $\mathrm{F}_{2}$ vapor could be designed, but this s.lternative was not investigated here. If helium is used as a buffer, the capital investment in gas can be reduced significantly from the case of krypton. Also, the halium boiling point is much lower than the other gases, and it cannot be liquefied at normal cryogenic temperatures. Hence a liquid-vapor fractionator is not an appropriate approach. In this case, a simple flash separation was investigated. Figure 3.2-3 shows the flow diagram for this scheme. The feed gas is compressed and refrigerated, so that fluorine, argon, and krypton condense in a single-step process. The flash wold be made at pressure to prevent freezing krypton. Analysis of this process has shown that only a 


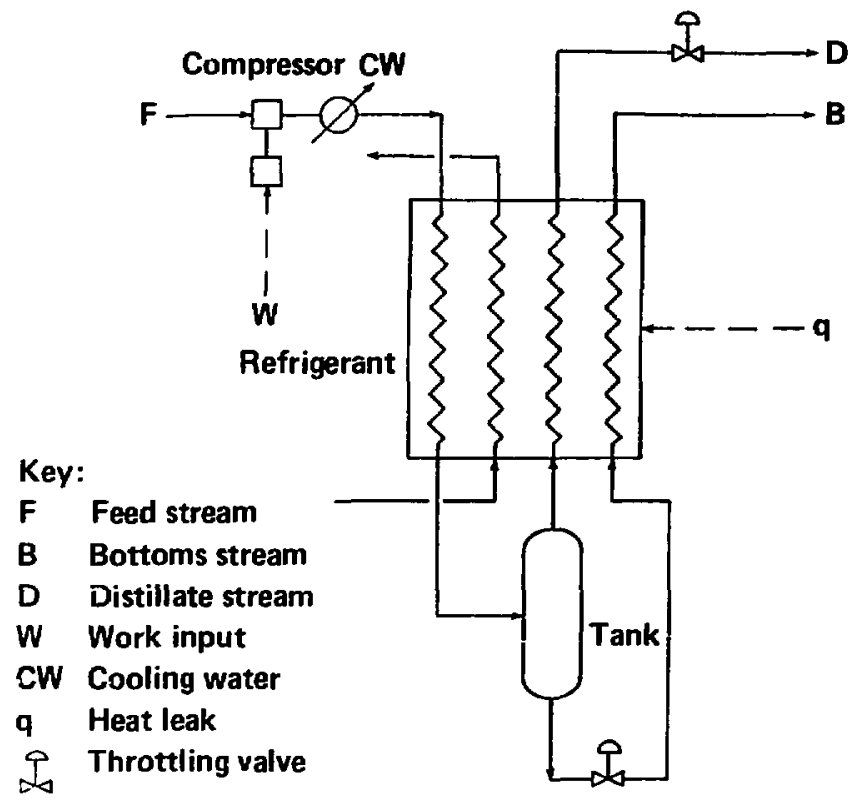

Fig. 3.2-3 Process-flow diagram for $F_{2}$ separation from helium buffer gas by flash separation. 
negligible amount of liquid is condensed, even at an inlet pressure of 700 psia and a temperature of $113 \mathrm{~K}$ (already below the freezing point of kryptcus. The isothermal compression power input alone at this pressure is $6.3 \mathrm{~N}$ per amplifier. Therefore this approach also appears impractical.

\subsubsection{Separation by Absorption}

Absorption of a component from a gas stream in a higher boiling liquid is a standard industrial separation technique. It is a particularly atiractive option in this case for these reasons:

- Absorption can be done at ambient temperature and atrospheric pressure if a suitable solvent is available for those condition.

- Only the minority component, fluorine, is renoved. The bulk of the gas need not be condensed.

- Absorption toes not provide sharp separations, which fractionation does provide. It will also remove some of the carrier gas. In this case, the sloppy separation is not harmful.

The disadvantage of absorption for this separation is that fluorine reacts with all normal solvents. About the only class of raterials that might be suitable are the totally fluorinated compounds, such as perfluoroheptane or hexafluorobenzene. These two compounds are stable, nontoxic, and nonflammable. Their boiling points are $82.5^{\circ} \mathrm{C}$ and $80.3^{\circ} \mathrm{C}$, respectively, which is lower than desirable but still feasible. Sulfur hexafluoride is also stable, nontoxic, and nonflammable, but it has a vapor pressure over 20 atm at $25^{\circ} \mathrm{C}$ and is ruled out for that reason.

Unfortunately, solubility data for the noble gases and fluorine in fluorinated solvents are not available. Lawrence Livermore National Laboratory, at Bechtel's request, has conducted a literature search and has found nothing. Without these data, a detailed design could not be made. Figure 3.2-4 is a flow diagram for a typical absorber-stripper combination. since this type of separation promises to be more economical than fractionation, a suitable solvent should be selected and solubility data obtiıned experimentally.

\subsubsection{Chemical Separation}

Fluorine is produced commercially by electrolysis of hydrogen fluoride in an anhydrous fused electrolyte, KF'2HF (melting point $71.5^{\circ} \mathrm{C}$ ), at 


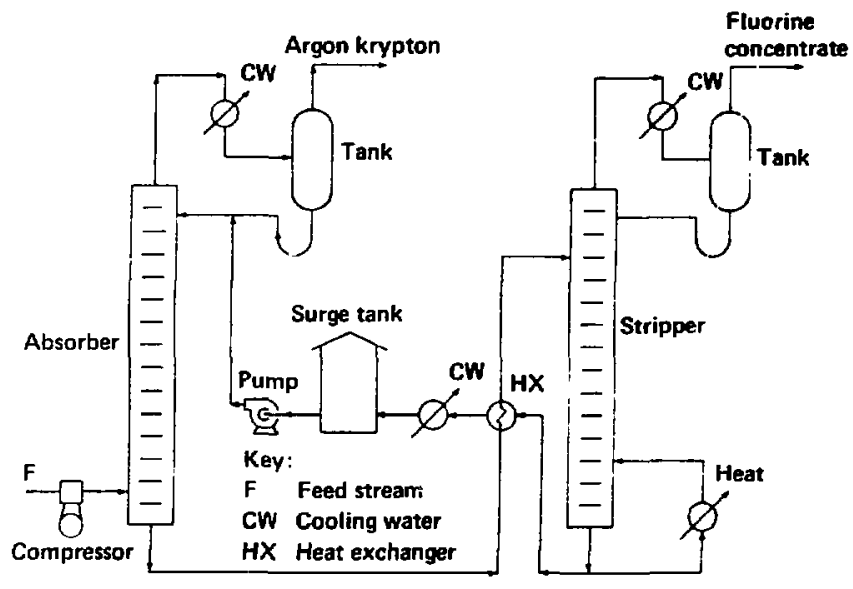

Fig. 3.2-4 Process-flow diagram for $P_{2}$ separation from the window buffer gas by selective absorption in a suitable solvent. 
90 to $95^{\circ} \mathrm{C}$. A standard production cell operates at a current of $6000 \mathrm{~A}$ with a voltage that varies $f$ rom 8 to $12 \mathrm{~V}$ over the life of the cell. The fluorine produced from the cell is contaminated with about 10 hydrogen fluoride and some carbon tetrafluoride. It can be purifled by refrigerating to $-140^{\circ} \mathrm{C}$, or by scrubbing with sodium fluoride. The standard cell described above produces slightly more than $1.0 \mathrm{~g}$ of $\mathrm{F}_{2}$ per second, for an average electrical power input of $60 \mathrm{~kW}$. It was estinated previously that each $\mathrm{KrF}$ amplifier will lose $17.6 \mathrm{~g}$ of $\mathrm{F}_{2}$ into the buffer gas per second. At this rate, the power required for electrolysis alone in a chemical separation system would be slightly greater thar $1.0 \mathrm{kN}$ per amplifier. It is possible that this figure could be sut in half if no buffer gas is used on the mirror side of the amplitier.

At first glance, this approach looks promising because the impact on overall efficiency would be much smaller than for the fractionation schemes discussed previously. The buffer gas stream to be purified would be mixed with hydrogen from the electrolysis cell to make hydrogen fluoride. The reaction should not be difficult to control at the low concentrations involved here. The boiling point of HF at atmospheric pressure is $19^{\circ} \mathrm{C}$, so that separation by condersation at low temperatures is much easier than for $F_{\boldsymbol{2}}$ $\left(\mathrm{bp}-188^{\circ} \mathrm{C}\right.$ ). However, it is estimated that the initial concentration of $\mathrm{AF}$ in the stream to be purified is only about 2000 ppm. Examination of the HF vapor pressure curve shows that at $-75^{\circ} \mathrm{C}$ and $500 \mathrm{psia}$, only about $30 \mathrm{~s}$ of the HF would be condensed. The power required for compression of the gas to 500 psia would be at least $5.7 \mathrm{~m}$ per amplifier at room temperature (using Eq. (3-12)). Cooling to lower temperatures would cause additional problems because HF freezes at about $-83^{\circ} \mathrm{C}$. It is possible that a system based on precipitation of solid HF $f$ com the gas at much lower temperatures and atmospheric pressure could be designed. This approach was not examined here and may warrant further investigation. In any case, residual $\mathbf{B F}$ in the buffer stream must be removed by other (most likely chemical) means.

Hydrogen fluoride can be removed from the gas by absorption in an aqueous solution of calcium hydroxide (lime water) to precipitate solid calcium fluoride. A process flow diagram based on this approach is shown in Fig. 3.2-5. The calcium fluoride, after filtration and drying, is reacted with s: Ifuric acid to regenerate hydrogen fluoride, which is then electrolyzed. The buffer gas would also require dehydration before being returned to the amplifier flow system. While the overall process appears 


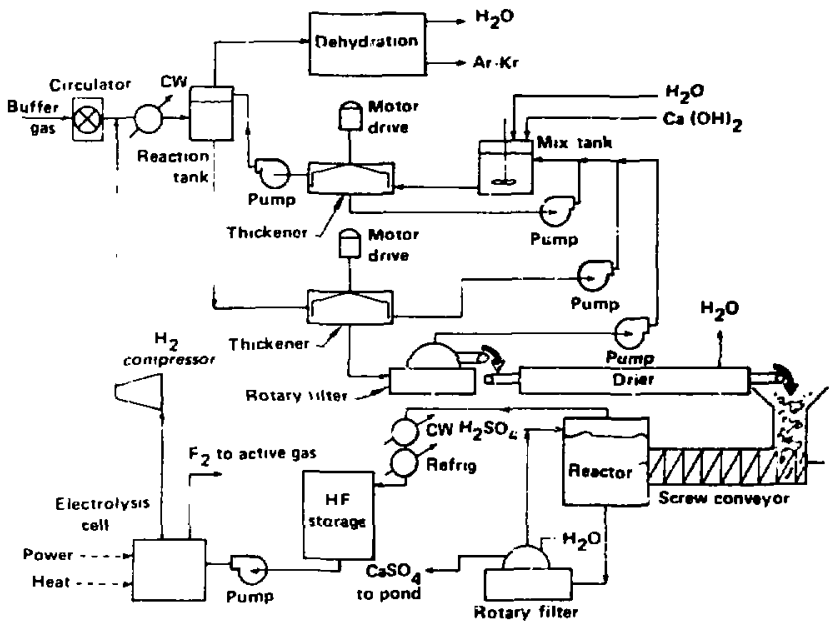

Fig. 3.2-5 Process-flow diagram for chemical separation of $F_{2}$ from the window-buffer gas. 
feasible, it is complicated and labcr-intensive, and requires the hanciling of some very corrosive chenicals. The idditional energy input to the process has not been determined, but is likely to be substantial. Furthermore, a waste stream of impure gypsum is produced in the reaction of calciu fluoride with sulfuric acid, and must be disposed of. For these reasons it is concluded that chemical separation appears unattractive.

\subsubsection{Conclusion on Fluor ine Separation}

All types of cryof ractionation systens studied were found to require very laige power input for compressors and refrigerators. Chemical cleasup was not investigated in significant detail, but also appears to be energetically expensive. The prospect of separating fluorine by absorption in a totally fluorinated liguid appears attractive, but no data on fluorine and rare gas solubility could be obtained, sc suitable designs could not be studied in detail. Since no acceptable separation system was identified, the concepts of aerodynamic windows and gas-buffered windows for the $\mathrm{Xr} P$ applifiers have been dropped from further consideration.

\subsubsection{Other Flow Design Considerations}

From the preceding sections it is apparent that the only reasonable design approach identified to date for the $\mathrm{KrF}$ amplifier is to use a highly pure Eused silica window that stands as close as possible to the active region. Analysis by Physics International (Appendix 1) incicates that at a distance of 20 to $25 \mathrm{~cm}$ fror the active region, and with a 1-kG magnetic guide field, heating by sicescattering from the e-beam will not damage the window or its coating. If the window and mirror are both placed $25 \mathrm{~cm}$ from the active region, then the total amplifier cavity measures $3.5 \mathrm{~m} \times 2 \mathrm{~m} \times 2 \mathrm{~m}$. At a aistance of $25 \mathrm{~cm}$, the loss due to absorption of fluorine in the inactive region will be $2.5 z$. The window at this distance must be thick $(\sim 10 \mathrm{crn})$ and probably segmented (four pieces, 1 × $1 \mathrm{~m}$ each is assured) in order to withstand the pressure wave. If the segments are supported by steel struts 5 cm wide, an additional loss of at least 58 due to their shadows must be accepted. Adding the absorption loss in the window and coating the total window brings optical losses to about 108. Gas flow systems specific to the A.r/K $\mathrm{K} / \mathrm{F}_{2}$ active gas mixture are discussed in the next few paragraphs. 


\subsubsection{Construction Materials}

At temperatures near abblent, dry fluorine is been handled satisfactorily in steel, stainless steel, nickel, and copper. The surfaces are apparently passivated by oxidation of a tnin surface layer. The low concentrations in the laser amplifier should cause no problems, and the preliminary choice of construction material is mild steel. It should be noted tirat the systen must be kept extremely dry because water vapor increases the corrosion of both the metal walls and quartz windows (if used) of the ampiffiers.

\subsubsection{Heat Removal}

If the full $1.44 \mathrm{MJ}$ of e-beam energy lost in the gas per shot is assured to go into gas heating, then the average temperature of gas entering the heat exchanger will be $160^{\circ} \mathrm{C}$. The cooling water inlet and outlet temperatures are taken to be $35^{\circ} \mathrm{C}$ and $50^{\circ} \mathrm{C}$, respectively, and the gas outlet temperature is $50^{\circ} \mathrm{C}$. The requiced cooling water flow rate under these conuitions is about 800 gallons per minute (gpa) per amplifier.

To reduce pressure drop, the exchangors are designed with the sane cioss-sectional grea as the ducts they connest to. The tubes are installed along the larger dimension (3.5 jil long) ans are finned to inprove the heat-transfer coefficient. The cooing water makes an equal number of passes across the duct in each direstion to minimize gas-temperature differences across the width of the duct at the exit. Making reasonable assumptions regarding heat transfer coefficients, but without detailed exchanger design, a total heat transfer area of $6000 \mathrm{ft}^{2}$ ior each amplifier flow $100 \mathrm{p}$ is estimated. A preliminary estimate of the pressure drop through the heat exchangers gave approximately $1 \mathrm{ps}$. It is felt, hawever, that a detailed design to minimize pressure drop could reduce this to 0.5 psi.

since the riplifier is firing twice a second, the temperature of the gas at the exchangez inlet will vary with some regular pattern. This temperature pulsation will be damped somewhat by the turbulence in the gas, and the exchanger itself will damp it more for two reasons:

- The tubes create turbulence and mixing by the alternate contractions ard expansions as the gas flows between them.

- Due to the increased temperature difference, the hotter gas will transfer more heat to the water than the cooler gas, thus leveling out the temperature cycle. 
In spite of these daping effects, it appears likely that there will be some temperature cycle at the exchanger outlet. some of the residual cyclic effects will be renoved by flow strean shearing around turns. Bopefully the remainder will be adequately elininated by the extrene turbulence in tho blower.

The control temperature should be measured downstrean from the blomer. It is possible that temezature uniformity of less than $1^{\circ} \mathrm{C}$ ay be required in the laser cavity to limit optical wavefront distortion by refraction in the medium. This may be difficult to do using existing industrial tecnnology. Further stujy of the exact process requi rements and potential solutions is needed.

\subsubsection{Pressure-Wave Damping and Flow Management}

The pressure wave initiated by the deposition of e-bean energy will reverberate in the ducts of the flow loop for some tine after each shot. If significant residual-gas density fluctuations still exist in the laser cavity at the time of the next shot, the wavifronty of the laser pulses will be distorted, and the increased beam divergence may reduce the energy incident on the Raman cell. Previous studies ${ }^{3,4}$ have shom tlis't residual density fluctuations can be a siạnificant problen when a pulsed laser is used to irradiate a small fusion pellet target (on the order of 1 in dianeter), especially when the time between pulses is short (high repetition rate). Neither of these conditions is obtained in the present study, and $\mathrm{n}$ adequate analysis of laser beam divergence under conditions appropriate to this system has yet been performed.

When a l-ram target is irradiated by a lasez from a distance or $100 \mathrm{~m}$ the target subtends an angle of $10 \mu \mathrm{rad}$, as seen from the laser. Iir order to efficiently couple energy to the target, the beam divergence mint be small compared to this angle. In the present system the longest optical path from the amplifier to the Raman cell is $400 \mathrm{~m}$ (including optical delays), but the effective target size is on the order of $1 \mathrm{~m}$ (the Raman cell apirture). Thus the Raman cell subtends an angle of $2.5 \mathrm{mrad}$, as seen $b y$ the amplifier. Therefore, the allowable KrF beam divergence in the Raman puise compreszed system is 250 times larger than for the previous example. To iliustrats, a

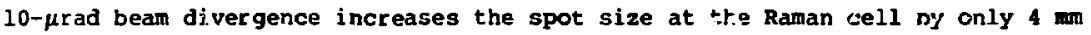

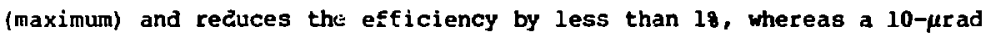
divergence would cause direct target irradiation to be cut by a factor of more than 2 . 
In addition, the interpulse tine is $500 w$ for the systex under consideration here. The transverse acoustic transit times for che duct with a , und-wave velocity of $325 \mathrm{~N} / 3$ are $6 \mathrm{~ms}$ and $11 \mathrm{~ms}$ for the $2-\mathrm{m}$ and $3.5 \mathrm{~m}$ dimensions, respectively. Mransverse acoustic nodes will undergo 60 and 45 reflections in the short and lang dimensions, respectively, between sbots. If oniy a 208 ceduction in applitude per bounce if assured, the attenuation factors would be $10^{7}$ and $10^{4}$ f $\mathrm{sr}$ sound waves rebounding betweer. shots in the short and long dimensions, respertively. These are felt to bu quite adequate attenuation factors and should be obtaiksble with passive sidewall absorbers. On the other hand, the longitudinal acoustic sransit time (around the flow circuit) is about $100 \mathrm{~ms}$, indicating only five transits betwren shots. However, the longitudinal path travels through the blower and heat exchanger, which will provide considerable camplng on each pass.

It is likely that some form of acoustic absorbers wil be necessary to provide the required transverse-wre attenuation. Provision has teen made for vented (perforated) sidewall absorbers on all sides of the duct for a distance of $4 \mathrm{~m}$ up and downstream from the laser cavity. If this type of muffler is not adequate, porous materials can be added along the walls onc/or in the side-jent cavities. It may also be useful to include some low-pressure drop-flow conditioning elements, such as flow straightenirg vanes, a honeycomb (an ar:ay of longitudinal hexagonal tubes), and perhaps ayen a screen, upstream irom the laser cavity to further reduce any density nonuniformity. None of these devices is expected to rad any significant pressure drop in the flow loop, and adequate medium homogeneity is expected. Further theoretical and experimental studies of the exact system requirements in this area, and how to achieve them, are needed.

\subsubsection{Pressure Drops and Blower Power}

The amount of gas circulated in each KrF amplifiez gas loop is very large (ahout $56,0001 / \mathrm{s}$ ). To reduce the power required to circulate this amount of gas, it is necessary to keep the pressure drops as low as possible. An estimate of the pressurt drops dua to various components in the flow channel is given by (see Ref. 3)

$$
v_{F}=K_{i} \rho \frac{v^{2}}{2}
$$

where $f$ is the gas delsity, $v$ is its velocity, and $k_{i}$ is a constant 
characteristic of the ith component. The gas velocity in this syster is rather $I O W$, so that $\rho v^{2} / 2$ is only $10^{-3}$ atm. The sum of all coefficiants, $k_{i}$, for components in this flow loop, outside the heat exchanger (screen, honeycomb, boundary layer friction, flow turns, etc.l, is on the order of 10 (seo Ref. 3), so that $\Delta \mathrm{p} \sim 10^{-2}$ ats is estimated for then.

Tre major pressure drop will occur in the heat exchanger itself. With careful design, it is felt that this drop can be limited to about 0.035 atm ( 0.5 psi). With inclusion of the other frictional losses, a total loop-pressure drop of $0.045 \mathrm{~atm}$ is estimated. The power dissipated in the flow loop is given by

$$
P_{F}=\Lambda p \cdot \frac{\Lambda V}{\Lambda t}
$$

where $\Delta \mathrm{V} / \Delta t$ is the volume flow rate. In this system the aissipated power is $200 \mathrm{~kW}$ per amplifier loop. If a blower efficiency of 0.7 is assumed, an input power of $370 \mathrm{~kW}$ would be required.

The blower can be a standard power-plant design. The only nonstandard item is lubrication. Standard petroleuni lubricants cannot be used because they will react with the fluorine gas. Either fluorinated lubricants must be used, or the machine must be $r$ un dry (carbon or teflon bearings).

\subsubsection{Conling Water Flow Power}

Typical industrial cooling tower head pressures run about 50 psi. At 800 gpm through the heat exchanger, the power dissipation is $17.4 \mathrm{kw}$ per amplifier. Twenty-five kilowat.ts of water pump power will be required for each amplifier, assuming 708 pumping efficiency.

\subsubsection{Laser Gas Cleanup}

The laser gas will inevitably be contaminated by impurities fram leaks, material outgassing, and from fluorine reactions. Air leaks introduce carbon dioxide and water vapor into the system, in addition to nitrogen and oxygen. Water is particularly hamful because it increases the corrosivity of the flur,rine.

Fluorine reaction products will accumulate in the system with time. Some of these reaction products are metallic fluorides from the duct work, valves, blower, etc., ana these will be present as fine particulates. Other comon fluoride compounds that may appear include carbon tetrafluoride, hydrogen 
fluoride, and the whole range of fluorinated hydrocarbons. Hany of these compounds are gases under the operating conditions.

Contaminant gases can be renoved by bleeding off a small stream of gas and removing all but the rare gases from it. Fluorine and oxygen can be removed by reacting with a hot metal. Most other molecular impurities (with the exception of nitrogen) can be removed with a cold trap. Finally, $\mathrm{N}_{2}$ can be removed by reaction with hot magnesi um, $3 \mathrm{Mg}+\mathrm{N}_{2} \rightarrow \mathrm{Mg}_{3} \mathrm{~N}_{2}$. The rare gases are then returned to the main flow loop with fresh fluorine from an external tank. Continuous monitoring of the gas composition will be necessary. External gas tanks will also be used to readjust the partial pressures of the rare jases. The cost of the gas maintenance system should be relatively small because the feed rate is small. More detailed design of th: cleanup system should be undertaken when more is known about the contamination rates sild the effects of the various contaminants on laser operation.

\subsubsection{Startup 2.2d Shutdown Operations}

A cost saving in the construction of the flow system is realized if it is not reyuired to be evacuated before being charged with the laser gas. In this case, the duct work will not need reinforcement to withstand the inward atmospheric pressure. A procedure for filling the laser flow loop that avoids vaculon pump down is then required.

The filling procedure can start with a purge of the system by argon gas. This should be done by introducing the heavier argon at the bottom of a flow loop and venting the lighter atrosphere (mostly $\mathrm{N}_{2}$ and $\mathrm{O}_{2}$ ) from the top. The vent from one flow loop should be connected to the inlet of the next. In this way, the total amount of argon vented to the atmosphere will be minimized. Krypton gas should then be introduced in proper proportion to each amplifier throught the bottom inlet, while venting argon from the top. Residual impurities in the gas system should te removed chemically before the fluorine is added. This could be done $t: y$ the systems designed for cleanup of the laser gas if the impurity concentrations remaining after purging are sufficiently low. The smal? fraction of fluorine required would be introduced as a last step before operation begins.

When maintenance on the amplifier system is required, it will sometimes be necessary to remove the laser gas and reintroduce a normal atmosphere to the flow loop. Fluorine should be chemically stripped fron the flow system as a first step. Argon and krypton could then be vented to the atmosphere. 
However, the cost of these gases will probably dictate that they be puped into storage for reuse (each i mplifier system will contain approximately $\$ 30,000$ worth of $\mathrm{kr}$ and as much $\mathrm{as} \$ 3,000$ worth of $\mathrm{Ar}$, depending on its purity\}. If the inert gases are to be stored, they should be reasonably well separated so that the subsequent filling procedure can be perforned as indicated above. Krypton should be renoved by a small cryogenic fractionator designed for this purjose. Argon or helium can ife introduced to maintain atmospheric pressure as the krypton is removed. The argon can then be displaced with helium, from which it is separated cryogenically and pumped into storage tanks. Finally, the helium would be venter while it is displaced by the normal atmcsphere the helfum gas lost by venting would be valued at less than $\$ 500$ per amplifier). Allowances for the necessary gas handing and storage systems have been included in the cost estinates for mechanical equipment.

\subsubsection{Flow System Layout}

Figure 3.2-6 is a layout of the KrF amplifier flow loop as envisioned at the end of this study. No provision is made for buffering the anplifier window or mirror witil a fluorine-free gas. Gas handling systems for startup, shutdawn, and cleanup are not shown. The heat exchanger is divided into two parts, one to remove heat added by the e-beam, and the other to remove heat ddded by the blower.

\subsection{Methane Raman Cell Gas Flow Systems}

The active region of the backward Raman pulse compression cell must be $15 \mathrm{~m}$ long, corresponding to half the length of the 100-r.3 KrF pump pulse. Further general design parameters for the Raman compressor established at the beginning of the study are given in Table 3.3-1. A backward first stokes gain of $10^{3}$ is obtained by double passing the pump and stokes beams through the cell. The geometry of the optical system shows that, with a reasonable distance between Raman cell mirrors (180 $\mathrm{m}$ end-to-end), the clear aperture must be approximately twice the output aperture. The Final design, therefore, allows for two separate cells side-by-side fone for earh pass of the pump and Stokes pulse), each with an aperture of $4 \times 12 \mathrm{~m}$.

High-purity uncoated quartz (e.g., suprasil) windows orfented at Brewster's angle are used to enclose the methane. The optical pulses are assumed to be linearly polarized to minimize reflection losses. The 

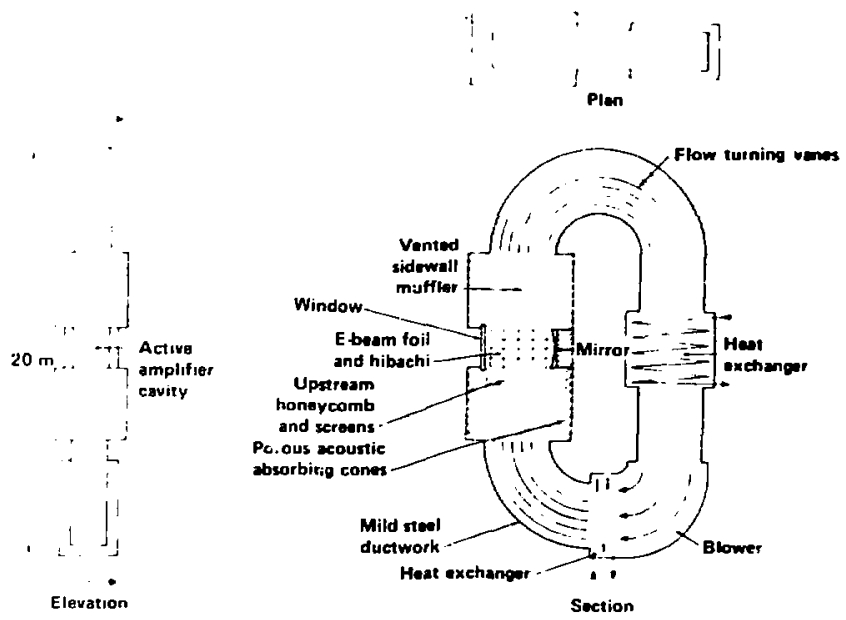

Fig̣. 3.2-6 KrF arolifier gas-flow system. 
Table 3.3-1

Methane Raman compresser design paraueters.

Stokes pulse langth

Extraction efficiency

Compression factor

pump pulse lensth, (cell lerigth)

Coll pressure

Stokes output fluence

KrF pump fluence

output eneroy

KrF pump ener qy

Output aperture $(2 \times 7$ beam array)
$20 \mathrm{~ns}$

808

$5 x$

100 ns (15 m)

1 atm

$4 \mathrm{~s} / \mathrm{cm}^{2}$

$5 \mathrm{~J} / \mathrm{cm}^{2}$

$1.5 \mathrm{MJ}$ (est.)

$1.9 \mathrm{WJ}$ (est.)

$3.3 \times 11.6 \mathrm{~m}$ 
Brewgter-angle or ientation also reduces the energy fluence on the optical surfaces by the cosine of the angle. In this case, the output window aces the maximum filuence of approximately $2.3 \mathrm{~J} / \mathrm{cm}^{2}$ at $248 \mathrm{~m}$ in $100 \mathrm{~ns}$, followed by $2.2 \mathrm{~J} / \mathrm{cm}^{2}$ at $268 \mathrm{~nm}$ in $20 \mathrm{~ns}$.

The vibrational energy depostted in the methane in the process of Ranan scattering raises the temperature and pressure of the medium only very slightly. Therefore, little acoustic damping is required, and the windows need not be thick (a l-cn thlckness is assumed). The arount of energy deposited in the methane, $\mathrm{E}_{\mathrm{d}^{\prime}}$ is given by

$$
E_{d}=E_{s} \frac{\iota_{r}}{v_{s}},
$$

where $\mathrm{E}_{\mathrm{S}}$ is the output stokes pulse energy, and $\nu_{\mathrm{s}}$ and $\nu_{\mathrm{r}}$ are the stokes and Raman Erequencies, respectively. Here $\nu_{5} / \nu_{r}=9.08$, so with $E_{s}=1.5 \mathrm{MJ}$, only $120 \mathrm{~kJ}$ is deposited in the methane. Approximately 858, or $100 \mathrm{~kJ}$ of this total is deposited on the second pass. For methane, $C_{v}=$ $3.2 \mathrm{nR}$, so the temperature $\mathrm{r}$ ise is given by the following (compare Eqs. (3-2) and $(3-3)$ )

$$
\frac{\Delta T}{T_{0}}=\frac{1 U}{3.2 P_{0} V_{0}}
$$

The active volume of the second Raman cell is approximately $600 \mathrm{~m}^{3}$, which at a pressure of 1 atm yields $P_{0} V_{0}=60 \mathrm{MJ}$. Using $\mathrm{Bg}$. $\left(3-16\right.$, with $T_{0}=$ $300 \mathrm{~K}$, the average temperature $\mathrm{r}$ ise in the second Raman active region is found to be less than $0.2^{\circ} \mathrm{C}$, and the corresponding preasure $r$ ise is only 0.4 Torr. If necessary, it is felt that the small pressure wave generated by the Raman scattering process can be adequately damped out by passive porous acoustic absorbing material on the inside walls of the Raman cell. Since the temperature $r$ ise is very small on each shot, the gas can be allowed to flow longitudinally along the cell. At a flow rate of $1 \mathrm{~m} / \mathrm{s}, 30$ shots $w i l l$ be fired at $2 \mathrm{~Hz}$ during the passage of the gas through the 15-m-long active region, with a net temperature rise of less than $5^{\circ} \mathrm{C}$ in the second cell $1 \leq$ $I^{\circ} \mathrm{C}$ in the first). The resulting longitudinal temperature gradient in the methane is not expected to degrade beam quality significantly (a lateral gradient would cause some beam steeringl. 
In the second Raman cell, a heat exchanger wust be provided in the return gas flow loop to remove $200 \mathrm{~kW}$ of heat. This can be done with the equivalent of a 700,000-Btu/h air conditioning system. Approximately another $100,000 \mathrm{Btu} / \mathrm{h}$ is required in the first Raman cell. 'The total heat exchanger surface area will be small, as will the corresponding pressure drop through it. This, combined with the slow gas flow velocity, will result in a rather small required input, power to the gas circulation blowers. It is felt that a few kilowatts of blower power will be adequate, which is a negligible addition to the overall system input power, Variations in the design of the methane flow system, including higher velocity transverse flow, would not increase the required blower power to a point where an impact on overall system efficiency would be felt.

Gas purity is not a significant issue in the Raman amplifier, because most comonly expected contaminants will not interact with the laser pulses. If natural gas is used as a source of methane, it may be necessary to remove heavier hydrocarbons cryogenically before filling the cell (ethane concentrations of several. percent would be anticipated). The cell can be filled by purging until the concentration of impurities is sufficiently low. During the rurge, excess methane can be vented to the atmosphere, or burned off as appropriate. Similarly, during operation a bleed stream may be vented or burned exterrally, while fresh gas is added to maintain adequate purity. The simple gas-handling systems required will have little impact on system cost and negligible impact on uverall energy-conversion efficiency.

\subsection{Overall System Energy Output: and Effictency}

The system has been designed with the goal of 160-kJ output energy from each final anplifier. At $2 \mathrm{~Hz}$ the total average power out of the 14 amplifiers is $4.48 \mathrm{M}$. Assuming an intrinsic efficiency of 108 and an e-beam sposition efficiency of $57 \%$, the input power to the dc power supplies must be $78.6 \mathrm{MH}$. The final amplifier output is degraded by the window losses (including free $F_{2}$ absorption) and amplifier fill factors (shadowing), in addition to output mirror reflectivities before pulse compression in the Raman cell. Overall losses--estimated in Table 3.4-1--result in an output beam after Raman compression with an average power of $2.63 \mathrm{~m}$, and a laser electrical efficiency of $2.63 / 78.6=3.38$. The output er.ergy per shot is $1.31 \mathrm{MJ}$. The output energy is considerably less than the design goal of $1.5 \mathrm{MJ}$. The primary reason is the inclusion of window losses due to $F_{2}$ absorption 
and the reduced fill factors resulting from the strut shadom in the faceted windows and amplifier housing shadows. Designs that occlude less of the active region with physical structures could inprove thia situation. At the game time, the input power is considerably higher than anticipated because of the low e-beam generation efficiency. It apgears likely that innovation in the e-beam generating systen could juprove on its efficiency.

T) estimate the overall system efficiency, the power used in auxillary equipment inust be added to that input to the porer supplies. The KrP amplifier blowers will consume approximately $5.6 \mathrm{kH}$ at $400 \mathrm{kH}$ aplece. The e-beam magnetic guide field systens use up $2.8 \mathrm{k}$, assuning $1.5 \%$ loss of energy in the resonant curcuit per shot. Pinally, the cooling tower water pums will use $0.7 \mathrm{M}$ if a 50-psi head pressure is assumed. The total system input power is therefore $87.7 \mathrm{k}$, and the overall efflaiency is $2.63 / 87.7=3.0$. The above est imates are also shown in Table 3.4-1. Thile this is considerably less than the 5 efficiency desired, it is hoped that improvenents in the design can substantially increase the efflciency.

\subsection{Structures and Auxiliary Equipment}

Synchronization of the pulses from each set of seven final amplifiers is ensured by scraping input pulses spatially from the output of a single preamplifier. The scraped pulses are then distributed radially outward to the final amplifier modules and recombined spatially after amplification at the central location.

\subsubsection{Stacker Beam Channel Design}

The layout of an individual stacker channel for a single final amplifier is show in Fig. 3.5-1. Input and output ray are shown. Detailed ray tracing for the stacker optles was shown in Figs. 2.1-1 and 2.1-2.

The segmented primary and tertiary telescope mirrors are set -ide by side in order to minimize the look angles into the amplifier and thereby maximize the $\varepsilon$ plifier flll factors. The distance from the anplifier secondary mirror to the primary/tertiary arrays is set by the requirement of $>95$ arulifier fill factors for all beams (neglecting window strut shadows). Here, a distance of $120 \mathrm{~m}$ was chosen, and should give $>95 \%$ fill for the outer beams and $>988$ fill for the inner beams.

The delay-generating and delay-removal mirrors are placed on opposite sides of the propagation channel. The unit magnification of the telescope 


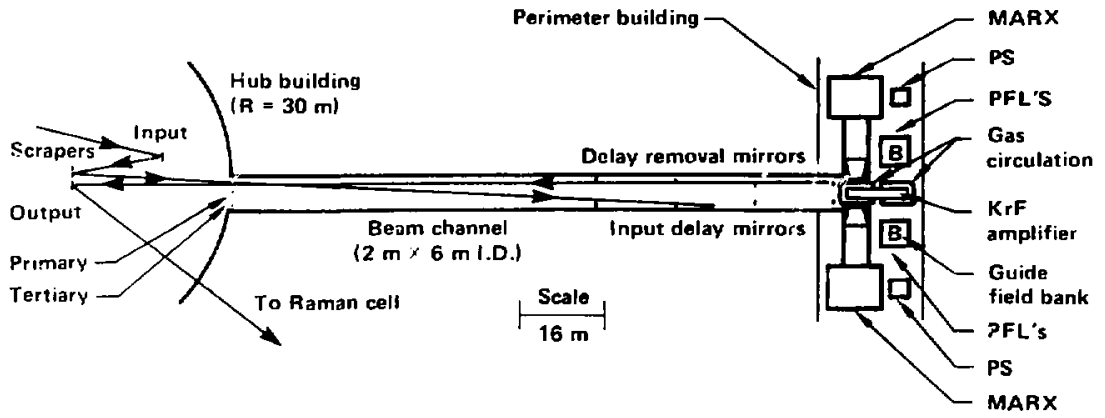

Fig. 3.5-1 Individual stacker beam channel. 
Table 3.4-1

Laser system output and efficiency.

Laser electrical efficiency

Source

Wall plug input

E-beam deposition

Intrinsic KrF medium

Windows and fill factors

Mirroes

Raman conversion

\section{Efficiency $(\underline{3)}$}

100

57

10

90

82

80
Power (min)

$$
78.6
$$

44. $B$

4.48

4.03

3.28

2.63

Total laser output

3.38

$2.63 \mathrm{~W}$

\section{$(1.3 \mathrm{~kJ} / \mathrm{shot})$}

Auxiliary equipment input power

Gas-flow blowers

$5.6 \mathrm{MW}$

Magnetic guide fields

$2.8 \mathrm{MW}$

cooling water pumps

$0.7 \mathrm{M}$ A

Total auxiliary inpue

$9.1 \mathrm{MW}$

E-beam wall plug input

$78.6 \mathrm{MW}$

Total system input power

87. $7 \mathrm{MW}$

Overall svstem efficiency $\frac{2.63 \mathrm{MW}}{87.7 \mathrm{MW}}=3.08$ 
requires rectangular beam-channel dimensions of $2 \times 6 \mathrm{~m}$. Some optical aberration results from using the spherical mirrors off-axis. This is minimized by minimizing off-axis angles and therefore requires that the delay mirrors (input and output) be as cloge to the amplifier end of the channel as possible. The separation between delay mirrors is 15 a in order to generate the required 100-ns time delays.

The iost compact beam channel was obtained by allowing the input and output beams to cross each other at the input/output aperture of the channel. The distance from the input scrapers and output mirrors to the beam channel entrance is defined so chat clipping of the input beams by the channel aperture is avoided. In this case a distance of $30 \mathrm{~m}$ is acceptable. Between the channel entrance and the perimeter building that houses the final amplifier systems, the channel is supported by steel I-beams rising from concrete pylons. Allowance is made in the area of the stecker delay optics for platforms with increased stability.

The channel walls are 1-cm-thick steel plate, welded leak-tight, with insulated siding. External steel ribs are included for cross-sectional rigioity. A ventilation system is included to circulate the atmosphere in the prcpagation path. Fiping is included to transport cooling water to the mirrors. Allowance has also been made for electrical lighting and instrumentation.

\subsubsection{Perimeter Anplifier System Housing}

The major components of the final amplifier systems are also shown in Fig. 3.5-1. The Marx-bank-driven PFL e-bean generators for $400 \mathrm{~ns}$, discussed in Appendix 1 , are shown on either side of the amplifier cavity. The gas-circulation system described in section 3.2 is also shown. Power supplies for the Marx bank charging at $\pm 50 \mathrm{kV}$ dc ace shown with appropriate scale, as are the capacitor banks for energy transfer to the magnetic guife flelds. A reasonable lateral spacing of the equipment dictates a building width of $20 \mathrm{~m}$.

The length of the PFLs and e-beam output tubes would necessitate a large angular separation between adjacent final amplifiers if they were situated in the same plane. Since this would lead to an excessive length for the perimeter building, it was decided to stack the pulse-forming systems, as shown in Fig. 3.5-2. A vertical separation of about $10 \mathrm{ft}$ between the top of the PFLs and adjacent Marx banks is reguired for maintenance accessibility. The distance from the bottom of the lowe--module blowers to the top of the 


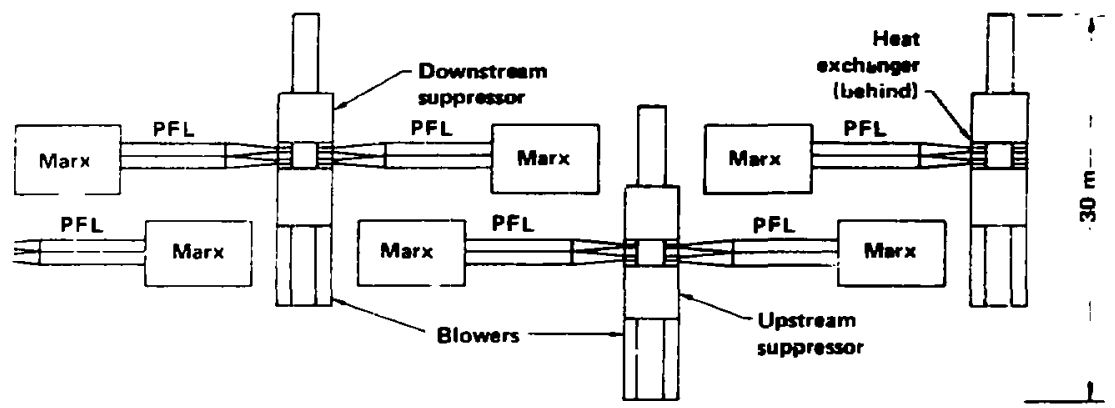

Fig. 3.5-2 Perimeter building elevation. 
upper-module ductwork is $30 \mathrm{~m}$, and the enclosing bullding is designed to be $35 \mathrm{~m}$ high to allow space for overheas cranes. With thi3 design the angular separation between beam channels is $11^{\circ}$, as shown in Fig. 3.5-3.

The perimeter building is assumed to be a simple Industrial metal fame structure. Sufficient structural steel for equipment surport and some: Interior finish has been allowed for. Additional equipent required ip the building includesthe following:

- A power distribution systen for approximate ly 90 we (78.6 Me of which is at $13.8 \mathrm{kVac}$.

- Cooling unjts for the PFL dielectric ater 14,000 cooling tons total system capacity).

- Heat exchangers for cooling the Marx bink oll.

- Other cooling water systems ttotal cooling water requirement is about $25,000 \mathrm{gpm})$.

- Marx oil piping to oil storage tanks.

- Safety gas purification system (carbon filter verit).

- Laser gas input, processing, and storage equipment.

- Miscellaneous - monorail holsts, sewer system, lighting, fire protection, process instrunentation, air conaitioning and heating systems, etc. No provision has been made for noise suppression, or for shlelding of the $x$ rays generated by the e-beam in the amplifier. These issues requrre further investigation.

\subsubsection{Hub Building Design}

For this study it was assumed that the building that houses the input scrapers and output mirrors (the "hub" building) extends radially outward to the channel entrances. This is a conservative design assumption because beam tubes could be extended inward behind the primary and tertiary mirror arrays to enclose the input/output beans and thereby reduce the building's radius. Further investigation of rinis possibiltiy would require a more detailed destgn of the input optical train, which is beyond the scope of this study.

The output mirror array is designed to combine the beams from the 14 amplifiers in a $2 \times 7$ stack, ylelding an output beam $3.3 \mathrm{~m} \mathrm{wi}: \times 11.6 \mathrm{~m}$ high (see Fig. 2,3-1(a)) The stacker channels must connect to tho hub at different heights to accomplish this, and are therefore slanted at different angles between the hub and perimeter buildings. The final amplifiers themselves must be correspcidingly slanted. In this design the primary and 


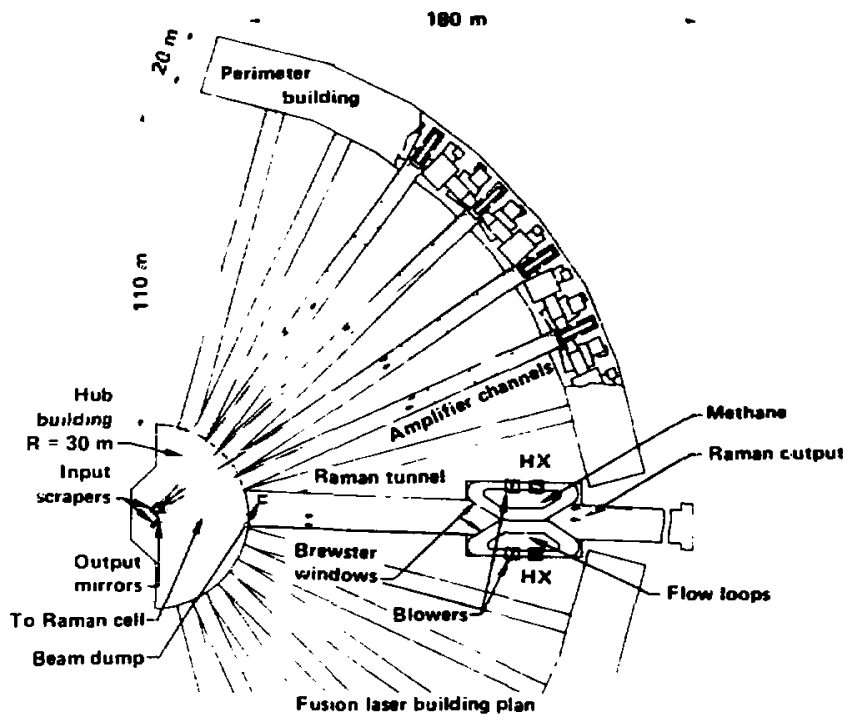

Fig. 3.5-3 Fusion laser building plan. 
tertiary stacker telescope mirrors are mounted at the entrance to the stacker channels at the outer wall of the hub. The helght of the building was conservatively taken to be $15 \mathrm{~m}$ to allow reasonable clearance above the various mireor arrays.

This building also contains the equipment for control of the atmosphere in the beam-propagation paths. In this case, the atmosphere has been assumed to be helium so that Rayleigh scattering of the short-wavelength laser light is kept to a minimum. The purity of the heiium need not be high. Contamination with. onsitutents of the normal atmosphere (primarily $\mathrm{N}_{2}$ and $\mathrm{O}_{2}$ ) will not produce significant losses until they reach about 108 concentration. For this reason the pressure in the beam paths may be kept slightly below atmospheric to minimize leakage of helium to the atmosphere. A system for maintaining adequately low impurity levels has been allowed for.

The structure consists of 1 -cm-thick steei plate welded leak-tight, with insulated siding supported by internal steel framework. A weldtd steel floor is also included. Allowances have been made for piping of cooling water for the mirrors, circulation of the atmosphere (ventilation), electrical lighting, and instrumentation.

\subsubsection{Ramai: Compressor Building Design}

The Raman compressor building extends outward between the two sets of seven amplifier channels. The length of the channel is determined by the requirement of adequate fill factors in the central methane cells. An overall length of $180 \mathrm{~m}$ has been assumed for this design. The two methane cells have a combined width of $8 \mathrm{~m}$ at the center, but the width of the channel at the ends must be $12 \mathrm{~m}$. This accommodates the pump input, stokes turning-mirror array, and beam dump at the hub end; and the stokes input optios, pump-turning mirror array, and output window artay at the other end. As a conservative assumption, the channel is taken to be $12 \mathrm{~m}$ wide throughout its length. The height of the channel is $15 \mathrm{~m}$ to allow some clearanci for the optics and the beams.

The struct;re consists of l-cm-thick steel welded leak-tight, including the floor. External steel supporting ribs are assumed for cross-sectional rigidity. The details of the Raman cell system are discussed in section 3.3. A cooling syster.l for the beam dump (1.6 $\times 10^{6} \mathrm{Btu} / \mathrm{h}$, or $400 \mathrm{~kW}$ ) is included to remove the heat generated by absorption of the remnants of the pump bearn. Aliowances have also been made for circulation of the atrosphere in the bean 
paths (ventilation), piping for cooling water to the mirrors, and beal dup, electrical power to methane blowers, lighting, and process instrunentation.

\subsubsection{Sitework}

The cost estimate incluces development of the external site. This includes 200,000 cubic yards of grading, $100,000 \mathrm{ft}^{2}$ of roads and paved areas, yard piping, and electrical lightirg. The estimate $\$ 150$ includes a cooling tower with $700 \mathrm{~kW}$ of pump power to handle the total 25,000 spa of cooling water.

\subsection{Facility Construction Cost Estimate}

\subsubsection{General}

The project consists of construction of a RrF laser system suitable for use as a target ignitor in an experimental inertial fusion test facility. This RrF Eusion laser system consists of the following:

- Twenty-eight e-bean generator systems, each depositing 0.B MJ of energy in the active Jaser gas in a pulse width of $40 \mathrm{C}$ ns. The e-beam systems include suitable Marx capacitor banks, PFLs, output switches, output lines, vacuum diodes (including anode foils), magnetic guide field generators, and appropriate power supplies.

- Optical systems consisting of mirrors, windows, mounts, ilignment systems, and mirror-cooling systems

- The laser front end, producing a 100-ns preamplifier pulse of $140 \mathrm{~kJ}$ total energy, for input to the final amplifier systems.

- Perimeter buildings, $20 \mathrm{~m}$ wide, $35 \mathrm{~m}$ high, with effective tota: length of $420 \mathrm{~m}$. The building houses the $14 \mathrm{KrF}$ final amplifier systems. The bulding is industrial-type steel frame construction with insulated metal siding and roofing on concrete mat foundations. Other equipment included in this structure is discussed in the previous section.

- No provision for personnel $x$-ray protection and noise suppression. It is assumed that personnel need not be present in the structure during operation. Since the laser system is part of a test facility, it is assumed that complete shutdown is permitted for any required maintenance.

- Fourteen beam channels, each $1.10 \mathrm{~m}$ long $x 2$ in high and $6 \mathrm{~m}$ wide. Channels were constructed of carbon steel plate with structural steel reinforcing and insulated siding. Channels are supported on steel supports and concrete footings. 
- Hub structure consisting of a carbon steel senicircular tank type structure on concrete foundations with insulated siding. Its radius is $30 \mathrm{~m}$, and height is $15 \mathrm{~m}$. The structure houses the central nirror assemblies.

- The Raman compressor bullding, 180 a long, 12 a wide, and $15 \mathrm{~m} \mathrm{high.}$ Construction is carbon steel plate with steel relnforcing, Insulated siding, and concrete slab foundation. A Ranan module with a nethane gas handling and cooling system is included, as discussed in a previous section.

- Site work consisting of grading an area of approximately $450 \times 300 \mathrm{~m}$; construction of approximately $10,000 \mathrm{~m}^{2}$ of paved area for roads; a 25,000-gpm cooling tower; site piping utility distribution for cooling water, fire water, potable water, and sanitary sewer; incoming elects ical power distribution; and yard lighting and grounding.

\subsubsection{Pricing Levels}

The estimate is at price and wage levels for the fourth quarter of 1979; no allowance has been made for future escalation.

\subsubsection{Direct Field Construction Costs}

The direct field construction costs of permanent plant equipment, materials, subcontrcts, and construction labor have been included in the estimate on the basis of the following discussion.

\subsubsection{Mechanical Equipment}

The cost of installed electron beam generator systens was furnished by physics International, as indicated in Appendix 1.

The cost of optical equipment, including the installation of that equigment, was furnished by Hughes Alrcraft Company, as indicated in Appendix 2. The $75.7 \mathrm{Ms}$ cost indicated in Table 2.4-1 does not include an allowance for general and administrative expenses and vendor fee. An estimated 258-- or 19.2 M\$-mas been added to account for these costs, bringing the total to $95.9 \mathrm{Ms}$.

The balance of the civil/structural and mechanical equipaent estimate is based on the equipment description furnished by Bechtel Engineering, as discussed in preceding sections of this chapter. 


\subsubsection{Materials}

civil/structural material costs are based on building areas and volumes, as applicable. Other direct material costs were factored from the civil/structural and mechanical equipment costs as appropriate.

\subsubsection{Construction Labor}

The manual labor rate was estimated at $\$ 16.00$ per job hour, including an allowance for casual overtime and craft-furnished supervision.

\subsubsection{Indirect Field Costs}

The indirect field costs that consist of temporary facilities, miscellaneous construction services, construction equipment and supplies, field office, preliminary check-out and acceptance testing, project insurance, taxes, and permits were included as a percentage of the direct labor costs; for this stady, 858 was used. A sunmary of estimated field costs for buildings and ancillary equipnent is given in Table 3.6-1.

\subsubsection{Front End costs}

The laser system front end provides tine inout pulses to the final anplifiers. The total energy output of the front-end subsystem, $140 \mathrm{~kJ}$, is approximately equal to the output of a single final amplifier pulse stacker (i.e., one-fourteerith of the output of all the stackers combined). To allow for the expected additional complexity of the front end, its cost has becn estimated to be twice that of a single final amplifier, or, approximateiy one-seventh (1.48) of the total cost of pulsed power, optics, buildings, and auxiliary equipment for the final amplifiers.

\subsubsection{Home office Services}

The home office services include engineering costs, other home office costs, and fre. Engineering includes preliminary engineering, optimization studies, specifications, detailed engineering, vendor drawing review, site investigation, and support to vendors. Other home office costs comprise procurement, estimating and scheduling services, guality assurance, construction and acceptance testing, and project managenent. Fee is included as a function of the total pruject cost. 
Table 3.6-1

Buildings and ancillary equipment coses (MS)

(400-ns pulsed power).

Givil/structural

$\begin{array}{lr}\text { Perimeter buildings } & 21.3 \\ \text { Propagation channels } & 10.4 \\ \text { Hub building } & 2.8 \\ \text { Raman channel } & 4.0 \\ & 38.5 \\ & 14.7 \\ \text { anical equipment } & 8.1 \\ & 4.1 \\ \text { lighting, and piping) dirgct } & 4.0\end{array}$

Mechanical equipment

4.7

Piping and instrumentarion

Electrical

4.1

Sitework (grading, paving, cooling tower,

4. 0

vard lighting, and piping) direce

Total direct field costs

69.4

Indirect field costs

17.8

Total field costs

87.2 
The sum of these three categories falls into historically consistent percentages in the range of 103 to $20 \%$, depending on the complexity of the project. For this study, a figure of $18 \mathrm{~s}$ of total buildings and auxiliary equipment, plus approximately 6 of vendor-installed equipment le-bean and optical systens) has been used. A figure of $30 \mathrm{ks}$ rounds off the total systen cost before contingency.

\subsubsection{Contingency}

Included in the estimate is a contingency that exists within the conceptual design in quantity, pricing, or productivity, and that is under the control of the constructor and within the scope of the project as defined. Inplicitly, the allowance will be expended during the design and construction of the project, and it cannot be considered as a source of funds for overruns or additions to the project scope. However, experience shows that it is guite difficult to assess the degree to which future processes are understood in the hardware sense. Thus, if the conceptual arrangerent of the plant crmponents contains majer uncertainties, or the design duty of plant components proves to be more severe than anticipated, or if additional major subsystens are ultimately found to be necessary, then the scope of the project is deened to have been inadequatley defined, and this then would not be covered hy the allowance.

The contingency for this study has been estimated at 258 .

\subsubsection{Design Assumptions}

Spread-footing type foundations can be used to support the structures and equipment.

A 100-W power source is available for start-up.

\subsubsection{Exclusions}

The following items have been excluded:

o Research and development costs for the laser system.

- Client engineering, financing, and other similar costs.

- Financing and interest charges during construction.

- Site investigation and land acquisition.

o Training of plant operators.

- plant start-up arid operation.

- Utillties and roads outsicie the site boundary. 
- Mobile equiprent.

- Pezsonnel radiation protection around e-bean generators.

- Noise-suppression construction around the e-beam generators.

- Common experimental test site facilities, such as site fencing, offices, warehouses, shops, central control room, power substations, etc. - Argon, krypton, fluorine, hellum, and methane gases required for operation.

\subsubsection{Estimate Summary (400-ns Pulsed Power)}

A summary of the overall construction costs based on the preceding discussion is given in Table $3.6-2$.

\subsubsection{0-ns System Costs}

A similar laser system based on 900-ns-duration pulsed power was investigated as a variation on the 400-ns system di.jcussed above. An underlying assumption in this wor's has been that the total energy output pel aperture remained the same, as did the number of final amplifiers. The major differences for the 900-ns system are that a PFN $e-b e a m$ generator is used and that nine beams are multiplexed to yield the 100-ns output pulse of the RrF stackers.

However, the underlying assumption of equal energy per aperture is quite unreasonable. The allowable size of a single $\mathrm{KrF}$ amplifier aperture is limited by parasitic-mode-control requirements, which depend in turn on the optical gain in the medium, and hence on the rate at which the medium is pumped. If the energy output of an amplifier were fixed, the pump rate would be reduced as the pump pulse is increased in length. The lower gain in the medium would then allow larger apertures to be built with adequate parasitic-mode control. Therefore, it is expected that a system with longer pump pulse width should allow a larger energy output per aperture, and hence a lower number of final amplifier units to produce the same total system output energy.

Nevertheless, a cost estimate has been made that assumes $14 \mathrm{final}$ amplifiers are used. The major design changes for the buildings result from the required increase in optical delay times and the decrease in the size of the PEN e-beam generator unit (relative to the PFL). Five additional delay-generating and delay-removal mirrors are required in each stacker channel. If one is taken to be $15 \mathrm{~m}$ closer to the hub building, with the other four farther away than the four mirrors of the 400-ns design, then the length of the stacker channels is increased by $60 \mathrm{~m}$. Taking account of an 
Table 3.6-2

KrF fusion laser system coses (Ms)

(400-ns pulsed power).

E-beam generator systems

98.7

optical systems

95.9

Buildings and auxiliary equipmen.

87. 2

Front end (148 of ahove)

39.1

Total field

321

Home of $\mathrm{fice}$

Total

351

Contingency 258

88

Construction cost, 4th guarter 1979

439 
Increased requirement for structural support of the additional nirror munts, the increase in clvil/structural cost of the channels is estiated to be 6.4 MS. Proportional increases are seen in the costo of nechanical, piping and instrumentation, and electrlcal systens for the channels. The reduction in size of the PFN e-beam generator results in a perimeter builaing reduced in length to $300 \mathrm{~m}$ (from $420 \mathrm{~m}$ ). Assuning the sane height and width for the perimeter building, the net civil/structural cost savings is estimated to be 5.7 MS. Piping and instrumentation in the perineter building are also reduced substancially because of the reduced size of the building. In addition, some reduction in indirect field costs reaults $f$ ran reduced labor charges. The sitework estimate remains the same because the total area of ground covered by the facility remains essentially unchanged. A sumary of costs for builaings and ancillary equipment for the 900-ns system is shown in Table 3.6-3. The overall cost is seen to be slightly reduced (1.5 Ms) by the indicated design changes.

With regard to the overall system costs, it was seen in Appendix 1 that going to a 900-ns PFN system saves a net $12.8 \mathrm{MS}$ in pulsed power. Analysis of the 900-ns optical system in Afpendix 2 1ndicates a net increase in cost of approximately 208 , or $19.2 \mathrm{M \$}$, in optical systems is required because of the increased number of components and system complexity. Front-end and home office costs change very little because of the small net change in costs from which they are factored. A summary of the overall custs for the system based on 900-ns pulsed power is shown in Table 3.6-4. A net increase of almost 10 MS is seen in tre total.

kgain, however, it should be noted that the actual design of a system based on 900-ns pulsed power would probably be based on fewer KrF amplifler modules of larger size. A net savings in all categorles would then be realized, and the overall cost would likely be reduced from the 400-ns case. Further investigation of the amplifier scaling relationships is required before such savings can be quantified.

\subsection{Sumnary of Technology Develogment Issues}

This study has been a detalled assessment of a particular initial point design that is not considered to be optimized. Further innovation 18 considered highly probable. Perhape the most significant result of this study has been the identification of important technical issues that must be addressed before a workable system can be built. A number of these issues are sumarized in this section, along with some propects for Improvements in system design. 
Table 3.6-3

Buildings and ancillary equipment coses (M\$)

(900-ns pulsed power).

Civil/structural

Perimeter buildings

15.6

Propagation channels

17.8

Hub building

2.8

Raman channel

4.0

39.2

Mechanical equipment

14.8

piping and instrumentation

6.5

Electrical

4. 1

Sicework

4. 0

Total direct field costs

68.6

Indirect field costs

17.1

Total field costs

85.7 
Table 3.6-4

KrF fusion laser system costs (M\$)

(900-ns pulsed power).

E-beam generator sistems

85.9

Jptical svstems

115.1

Buildings and ancillary equipment

85.7

Front end (148 of above)

40.1

Total field

327

Homr of $\mathrm{f}$ ice

30

Total

357

Cont ingency 258

89

Construction cost, 4th quarter 1979

446 


\subsubsection{ETF Design Philosophy}

It has been assumed that the laser system under consideration here is designed for use in an experimental test facility (ETF). This assumption is primarily evident in the Mar $x$ bank capacitor shot life $\left(10^{7}\right.$ shots). Bowever, it is important to note that the ETF design philosophy has not been rigorously applied in all areas.

A potential tradeoff between econory and efficiency exists that has received little attention to date. That is, for an ETF systen, we will not be interester as much in the ultimate laser syster ef $\bar{x}$ iciency as in the cost per unit of output energy. For example, the design allows for heliur gas in the propagation channels, including steel walls welded leak-tight and atmosphers circulation and maintenance systems. If the channels were filled with air, the system could be built more cheaply, but Rayleigh scattering would reduce the efficiency by somewhat less than 108, and the system aligment might be less accurate, resulting in added inefficiency. However, it might be more economically effective to build additional amplifiers to make up for the lost energy and accept the additional inefficienzy.

The same philosophy might be applied to the look angle and fill factors for the stacked beams in the Einal amplifiers. If the propagation channels were shortened, the fill factors would be reduced, resulting in lower system efficiency. However, the construction cost per unit of energy output wi'l be a function of the stacker channel length and will be a minimum for some finite length that has not been determined. If efficiency is not the most critical issue (and it may not be in an ETF), shorter channels might be used to reduce the cost.

A design study aimed at minimizing the system cost per unit of output energy would be useful. Such a study should be undertaken concurrent with work aimed at technical improvement of the baseline design.

\subsubsection{KrF Final Amplifier Design}

The final KrF amplifiers have been designed with 10-cm-thick, segmented fused silica windows in order to withstand the pressure wave generated by the e-bear energy deposition. Analysis has shown that this design would be adequate under static pressure conditions, but the effects of dynamic loading and repetitive operation have not been investigated in detail. Further analysis of the window's structural integrity and lifetime is needed. Such work would 
also serve to define more accurately the required fused silica window thickness, which has a strong inpact on the optical systen costs (see rable 2. 5-1).

The segmenting of the amplifier window ?eads to additional optical losses due to the shadows of the window-supportirg struts. Hew aplifier designs should be sought that minimize prolalens with the windows. It is evident that the amplifier aperture need not be square. The distance between ebean folls is limited by requirements on parasitic-mode contr $\gg l$, as is the amplifier length. However, the amplifier width in the flan , trection could be considerably longer because there are virtcally no reflective elenents on the flow sides of the cavity. A $1-x$ 4-m clear aperture couid produce the sane amount of output energy as the 2- $x$ 2-m aperture, but a segmented window could be designed for less optical loss in the former case. Designs which scale the amplifier up to a maximun al lowable length in the flow direction while reducing the e-bean foll separation should be investigated. It would also be useful to inves:igate designs in which the beans pass through the anplifier windows at Brewster's angie, thereby eliminating the necessity for damage-resistant antireflection coatings on those windows.

Further investigation of aerodynamic windows may be warranted if flow geometries or concepts can be found that substantial $+y$ reduce the anticlpated mixing of the active-medium gas with the window gas. The separation of Eluor ine from the window gas by selective abeorption in a totally fluorinated liquid ap.lears to be an attractive approach. Further investigation of the relative solubilify of $F_{2}$ and $r$ are gases in solvents such as perfluoroheptane and hexafluorobenzene is necessary in order to evaluate such a separation process.

The potential for e-beam-induced damage to the anplifier's wincow and mirror needs further study. This determines the required standoff distance between the optics and the active medium, which in turn affects the losses due to $\mathrm{F}_{2}$ absorption in the inactive region. The effect of magnetic-field fringing on electron trajectories at the ends of the amplifier cavity must be included in this analysis.

The effectiveness of pressure-wave actenuation by vented sidewall mufflers proposed in section 3.2.4.3 requires further analysis. When a shot is fired, residual ordered-density inhomogeneities created by the previous shot may Jimit the bean quality and efficiency of coupling to the Raman cell. Previous analysis done at $\operatorname{TRH}^{3}$ is inadequate because 1 t does not deal effectively 
with targets as large as the Raman cell. The assuption in the present work has been that the large size of the Ranan cell as a target, combined with the long interpulse tine ( $500 \mathrm{~ms})$, makes the vented sidewall auffler a viable approach.

The effects of going to longer punp pulses to take advantage of cheaper PFN pulsed-power technology are not adequately represented by this sludy. The principal reason for this is that the variation of optimum inplitier size as ? function of the punp pulse duration has been neglected. It is liksly that the permissible total energy per aperture will increage with increased pulse width because of relaxed requirements for suppression of parasitic moces. Therefore, fewer amplifiers would be required to produce the same sutput energy. Future studies must take this into account.

\subsubsection{Optical System Des.'gn}

It has been assumed in this study that losses due to Rayleigh scattering and reduce: a:ignment accuracy prohibits the use of a normal atmosphere in the beam-propagation channels. The use of helium gas as a beam-propagation medium requires construction of channels that are welded leak-tight. A cursory examination of the alignment system indicates that it may be overdesigned. That is, significantly more misalignment of the KrF beans can probably be tolerated without a significant raduction in system efficiericy. Therefore the argurent thr.t heliwi must be used to achieve adequate alignment accuracy is questicnable.

It has been noted prev.uusly that the losses due to Rayleigh scattering may be t.slerable when conssdered from an tconomic point of view $\{i . e$. , cost per unit of output energy). Furthermore, the contributions of separate atmospheric components $\left(\mathrm{N}_{2}, \mathrm{O}_{2}\right.$, and $\left.\mathrm{Ar}\right)$ to the Rayleigh-scattering attenciation in ai $\mathbf{r}^{5}$ have not been investigated. It is possible that one or more of these or other gases has acceptable low abosrption and scattering cross sections and would be suitable as a propagation medium. The use of any gas other 'han helium would reduce facility consruction costs because of relaxed requirements on leakage. The use of dry nitrogen is likely to yield the least expensive system.

Considerable improvements in the optical system design may be expected through Eurther innovation. One possibility within the framework of this design is to generate the stacker-pulse time delays in the preamplifier. This would allow elimination of the input time-delay generating mirrors and the 
tertiary telescope mirrors in each stacker channel. The final amplifiers would then be illuminated directly from a set of mirrors in the vicinity of the input scrapers. The number of mirrors would be reduced considerably, as would the beam-clannel dimensions.

Passing the entire pump beam through the Raman module twice does not appear to be an optimum design. The 5\% attenuation of the pump beam at the pump turning mirrors ${ }^{\left(M_{8}\right.}$ in Fig. 2.1-3) is unnecessary. A better design would probably result from amplification of the stokes bean in separate stages with expanding optics in between, followed by a single pass through the final backward Raman amplifier. It also appears that little if anything is saved in the present system design by combiring all the final Raman amplifiers in one unit, because this necessitates the construction of an additional long propagation channel. Designs should be considered in which individual final backward kaman amplifiers are situated in each stacker channel. The output of all the channels would then be recombined at the center of the hub building for final direction to the target. The reduction in the number of mirrors in the optical train would result in lower cost and higher system efficiency. A conceptual layout of a single amplifier channel incorporating same of these ideas is shown ir. Fig. 3.7-1.

Finally, a mirror reflectivity of 958 has been assumed in this study. The possibility of attaining higher reflectivities with dielectric coatings having adequate damage resiscance deserves considerable attention.

\subsubsection{Pulsed-Power Systen Design}

The efficiency of energy conversion from "wall plug" to e-beam deposition in the laser gas medium is projected to be $57 \%$ for the present design, as indicated in Fig. 1.2-2. The large inefficlency in this system suggests that innovative technology could result in significant improvements.

For example, a major source of energy loss (about 5-68) occurs in the shunt conductance of the dielectric water. in the PFLs. The water has been zssu... $d$ to be at room temperature. If the temperature of the water is dropped, its resistivity increases, and losses can be roduced to perhaps 1 or 28 (see Ref. 6) at temperatures near the freezing point. Because of the resistive losses in the dlelectric water, the water must be cooled to allow repetitive operation. Simple heat exchangers will not be acceptable because this would allow the dielectric water to $r$ un above ambient temperature and increase the losses. Hence cooling units (refrigeration units) for the 


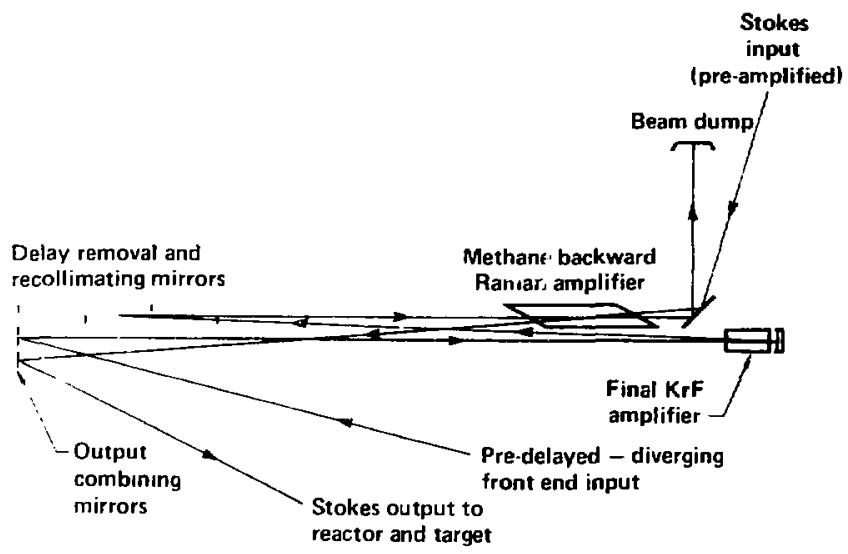

Fig. 3.7-1 Alternate stacker design using pre-delayed front-end input and individual Raman amplifiers for each stacker module. 
dielectric water have been included in the plant design to keep the water down to ambient temperature. If the cooling units are permitted to reduce the water temperature to well below ambient (perhape to near freezing), then the overall system efficiency can bo increased. In fact, because this reduces :he shunt-resistive losses, it may well have the effect of reducing the load on the cooling units themselves.

Looking beyond the EFF, it may also be possible to gain in energy-conversion efficiency between the reactor. and the storage capacitors. Here the normal conversion sequence goes as follows: thermal energy mechanical energy $\longrightarrow$ ac electricity $\longrightarrow$ dc electricity - capacitive storage. In Fig. 1.2-2 it is estimated that a 68 loss occurs in ac-dc conversion, plus another 28 loss arises in capacitor charging. If the reactor output is to be used to generete electricity, the question arises: Why not generate dc electricity directly to eliminate the required power supplits? If part of the reactor's thermal energy were used to drive a separate high-voltage dc turbine, the "wall plug" to capacitor loss might be substantially reduced.

Along the same lines, the dc machine could be a homopolar generator. Such a pulsed dc machine might be combined with an inductive intermediate-energy-storage system (instead of the Marx bank) that could then be used for PFL charging. This radically different design would require significant technical development, including the development of high-voltage opening switches, but major reductions in system cost and increases in system efficiency might be obtained.

\subsubsection{Other Facility Issues}

There appears to be no other significant issue associated with the construction of the facility as it has been degigned. It has been previously mentioned, however, that no provision has been made in the perimeter building for shielding of personnel from $x$ rays generated in the e-beam pumped amplifier. In addition, no acoustic noise suppression has been allowed for outside of the laser gas ducts. The rationale for this approach is that personnel will not be present in the structure during operation of the ETF. A complete shutdown of the facility is assumed for any required maintenance. For fluture commercial applications these assumptions may not be valid, and the issue deserves further scrutiny. 


\subsection{Conclusions}

A I int design for a laser fusion driver suitable for use in an ETF has been established and evaluated. The general concept is that of a hybrid KrF pulse stacker-backward Raman pulse compressor. The design is not considered optimized and, in fact, several suggestions for improvenents are $r$-de in the previous section. A few technical advancements or design innovations such as the development of long-shot-life switc ${ }^{*}$.es and high-danage-threshold optical coatings are needed before the system can be built as described, It is possible that design innovations such as the use of Brewster angle transmission throughout could elfminate the optical damage issue in the long run.

Tho possibility of using aerodynamic or inert gas-buffered windows for th? KrF amp'ifiers was studied in detail. However, it was found that considerable mixing of tro active and buffer gas streams will result from the e-beam-generated overpressure. Furthermore, no economical or enargetically efficient way of removing fluorine fron the window gas was found. The final design therefore uses solid fused silica windows close enough to the active region to reduce $F_{2}$ absorption losses to reasonable levels. In the present desıgn, the window must be thick (about $10 \mathrm{~cm}$ ) and faceted to give it the strength reguired to withstand the e-beam-generated pressure wave. The large thickness makes fused silica the most expensive line item in the optical system costs. Antireflection-coating technology for this window must be developer.

A coriceptual design for the KrF gas-flow system was presented, including startup, shutdown, and gas-cleanup systems. Provision has been made for passive pressure-wave suppression by vented sidewall mufflers with acoustic absorbing devices outside the vents. It is felt that this approach is adequate because a rather large beam divergence is tolerable without significant loss of efficiency and because the interpulse time to $500 \mathrm{~ms}$ is sufficiently long. The largest pressure drop in the flow loop will occur in the heat exchanger and may be kept as low as 0.5 psi. The blower power required to circulate the gas in each amplifier would then be about $400 \mathrm{~kW}$.

Brewster-angle windows are used for the methane Raman compression module so that optical-coating technoiogy is not an issue. The pressure drop in the methane gas Flow loop is low because orly small heat exchangers are necessary, and the gas can be circulated at a low velocity. Blower power for this flow loop makes a negligible impact on total system efficiency. Methane gas 
cleanup is not an issue because nost impurities do not interact with the beam. A small bleed stream can be burned off or vented so that fresh gas can be input, if necessary.

The total energy output in the stokes bean is $1.3 \mathrm{~m}$, with an average power of 2.63 . The overall system efficiency is estimated to be 32. Innovations in the pulsed-power and optical systens design way improve this performance significantly in the future.

Buildings to house the 400-ns pulsed-power laser gystem have been designea (conceptually), and a cost estimate for construction was made. The channels for beam propagation contain helium gas and are made of steel plate welded leak-tight. Construction costs could probably be reduced significantly if it is found that another gas can be substituted for the helium. When the costs for the pulsed power, optical systems, other process equiment, and buildings are added, and allowances are made for construction of the laser front end, home office costs, and contingency, the total system cost is estimated to be $439 \mathrm{MS}$ (last quarter 1979 dollars). It should be noted that the design has not been optimized in terms of cost per unit of energy output.

A system based on 900-ns pulsed power was considered as an alternative, and costs were estimated. It was found that increasea in the cost of the optical system are likely to be larger than the reduction of costs for pulsed power and buildings, so that tue overall system cost is slightly higher. However, this assumes that the same number of RrF ampliflers is needed in the 900-ns system. This is probably not true because requirements for the control of parasitic modes in the amplifier are relaxed, and larger single amplifier units can be built at 900 ns. Thus it appears likely that further cost reductions in optical systems and buildings would result in lower overall system costs for the 900-ns case. This tradeoff requires further study.

A summary of technical issues needing resolution before the system can be built was given in the preceding section. Cons:derable work remains in evaluation of suggested design improvements and in system optimization, 


\subsection{References}

1. R. A. Haas, Lawrence Livermore National Laboratory, private communication (1979).

2. D. A. Pinnow, T. C. Rich, F. W. Ostermayer, Jr., and M. Di Donenioo, Jr., Appl. Phys. Lett., 22, 527 (1973).

3. J. Shwarti, V. Kulkarny, D. Ausherman, B. Legner, B. Bobitch, R. Yano, and B. Sturtevant, "Fluid Mechanics of Fusion Lasers," mid-tera report for perod $9 / 11 / 78$ - 3/15/79, prepared for DOE Office of Laser Fusion under DOE Contract DE-AC08-78DP40070, TRH Inc. Report No. 33578-6001-RU-01 (5 Junz 1979).

4. H. 13. Hogge and 5. Crow, "Flow Design Concepts for Highpower Pulsed Visibie-Wavelength Lasers," final report for period 4/1/77 - 2/28/78, prepared for the Defense Advanced Research Frojects Agency and the Naval Research Laboratory, under Contract N00173-76-C-0239, Poseidon Research Report No. 16.

5 W. Eppers, "Atmospheric Transmission," in CRC Handbook of Lasers, R. $J$. Presstey, Ed., CRC Press, Cleveland, 1971, chapter 3, P 41.

6. J. 's. Benford, private communication. 